Portland State University

PDXScholar

Winter 3-10-2016

\title{
"Like Drinking Water Out of a Fire Hydrant" Medical Education as Transformation: A Naturalistic Inquiry Into the Physician Assistant Student Experience
}

Patricia Kenney-Moore

Portland State University

Follow this and additional works at: https://pdxscholar.library.pdx.edu/open_access_etds

Part of the Educational Psychology Commons, Educational Sociology Commons, and the Higher Education Commons

Let us know how access to this document benefits you.

\section{Recommended Citation}

Kenney-Moore, Patricia, "'Like Drinking Water Out of a Fire Hydrant" Medical Education as Transformation: A Naturalistic Inquiry Into the Physician Assistant Student Experience" (2016). Dissertations and Theses. Paper 2711.

https://doi.org/10.15760/etd.2707

This Dissertation is brought to you for free and open access. It has been accepted for inclusion in Dissertations and Theses by an authorized administrator of PDXScholar. Please contact us if we can make this document more accessible: pdxscholar@pdx.edu. 
"Like Drinking Water Out of a Fire Hydrant”

Medical Education as Transformation: A Naturalistic Inquiry Into

the Physician Assistant Student Experience

by

Patricia Kenney-Moore

A dissertation submitted in partial fulfillment of the requirements for the degree of

Doctor of Education

in

Educational Leadership: Postsecondary Education

Dissertation Committee:

Janine Allen, Chair

Christine Cress

Andrew Job

Gwen Shusterman

Portland State University

2016 
(C) 2016 Patricia Kenney-Moore 


\begin{abstract}
Physician assistants are medical professionals educated in an allopathic medical education model in the United States. In order to successfully matriculate, educate and graduate safe and effective health care providers in a 2-year time frame, the 4-year M.D. curriculum has been abbreviated and condensed leading to an intense, full-time cohort educational experience that taxes physician assistant students to their limits. The demanding workload can lead to fluctuations in mood and morale along with increased levels of psychological distress. This dissertation explores this under examined student experience by first introducing the physician assistant profession and the process by which it educates its members. The cohort patterns of mood and morale observed by faculty during the educational process are described using the conceptual and theoretical models of transformative learning, transition, change and cross-cultural adaptation as explanations for the observed experience. A retrospective naturalistic research paradigm utilizing focus groups elucidated the student perspective of the cohort medical education experience over the course of the didactic curriculum, and study results highlight a threestage experience consistent with stages-of-change theories from multiple disciplines. In addition, a prominent pattern of emotional subthemes provide a window into the psychological significance of this transformative experience. A better understanding of the effects of this academically rigorous and psychologically challenging medical education process on physician assistant students clarifies opportunities for amelioration of student challenges while simultaneously enhancing the ultimate goal of developing safe and effective health care providers.
\end{abstract}




\section{Acknowledgements}

This has been a long journey made possible only by the grace and support of my family, friends, coworkers and students. Your patience and encouragement has not gone unnoticed and I appreciate the many opportunities you have afforded me to selfishly explore my curiosity about my own experience as well as that of my students. Thank you from the bottom of my heart.

To my husband, Sam-thank you for your support in my seemingly never-ending academic endeavors. I would never have been able to work and attend school for the majority of our 35 years of marriage without your understanding. I have joked about "a degree every decade” often, and want you to know that my achievements are due to your longstanding patience. I look forward to weekends with you without writing!

To my program director of more than 20 years, Ted Ruback-thank you for taking a chance on me and giving me the space to grow into my skin. This dissertation would never have been a consideration without the opportunities you have provided to me during our longtime collaboration. My professional successes are directly attributable to you and your ongoing mentorship.

To my esteemed colleague, Bob DeBarge-your unwavering dedication to excellence has been an inspiration and I value our partnership in guiding students through the didactic year of our program. Thank you for willingness to share your personal time during the data collection phase of this project and for your valuable editorial expertise with early drafts of this work.

To Katherine Forney, thank you for your expert assistance with the graphics. 
My appreciation goes out to Dr. Janine Allen and my committee members for their wisdom, their patience and their counsel throughout this entire process.

And finally, to the more than 500 physician assistant students I have been honored to work with over the past 20 years at Oregon Health \& Science University-you have taught me far more than I ever could have taught you. I am forever indebted to each of you for your tolerance and flexibility as I continue to learn how to be a teacher. 
Table of Contents

Page

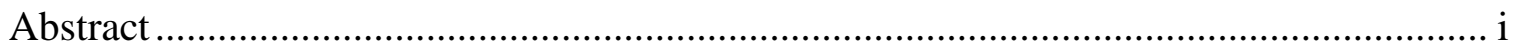

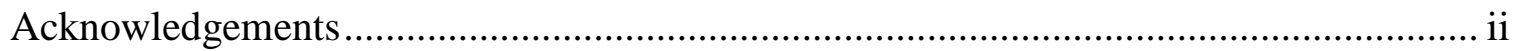

List of Tables ..................................................................................................... viii

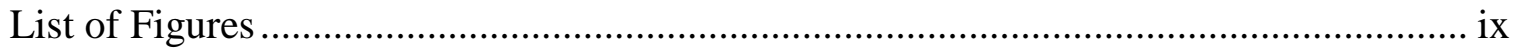

\section{Chapter}

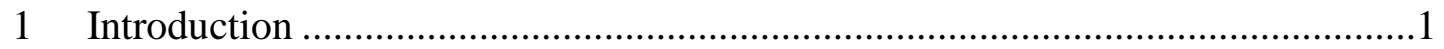

Definition of Terms...............................................................................

Overview of the PA Profession..............................................................8

Anecdotal Observations of Patterns of Student Morale...........................12

Significance of This Project..............................................................15

Research Questions to be Addressed ..................................................18

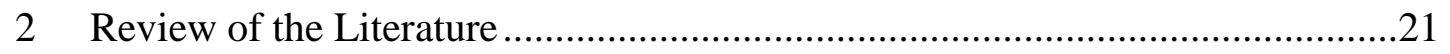

Identity and Professional Development ................................................29

Transformation, Transition and the Change Process ......................31

Transformational Learning ............................................................45

Mediators of Psychological Distress in Medical Education .....................48

Psychological Distress During the Process of Medical Education ............49

The Relationship Between Psychological Distress, Empathy and Professional Behavior ................................................................................50 


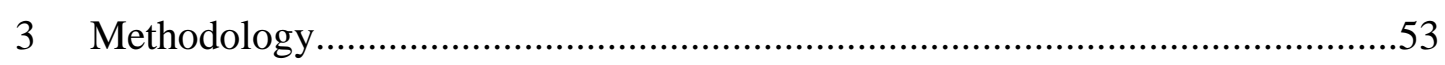

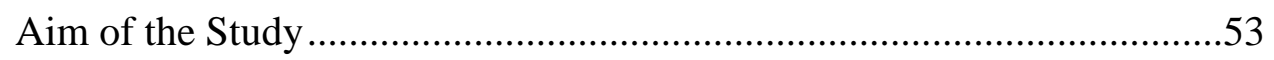

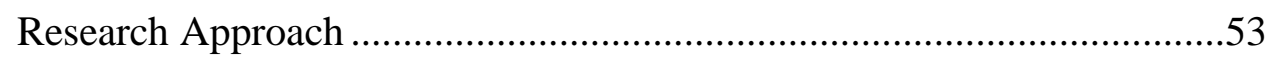

Study Participants ........................................................................................

Institutional Review Board Approval and Informed Consent .....................58

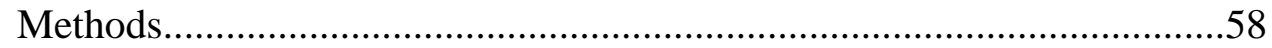

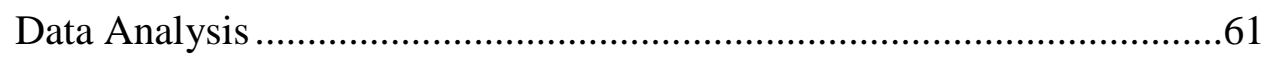

Methodological Considerations and Potential Research Bias ....................63

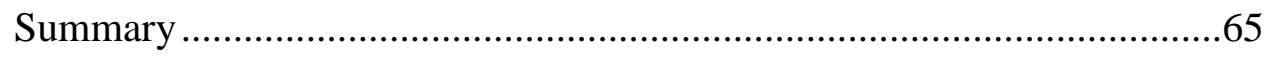

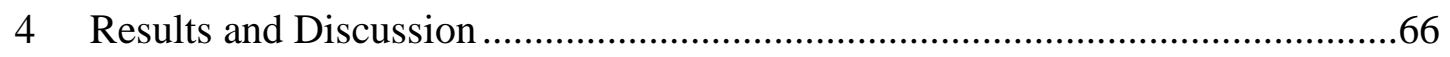

Content Analysis Themes and Subthemes .................................................67

The Experience of the Year Overall ...........................................................69

Transition, Change and Transformation: A Three-Stage Process With Emotional Implications ...................................................................73

The Beginning: "I Was Ecstatic ... At First”................................................76

The Middle: “It Was Overwhelming”.........................................................81

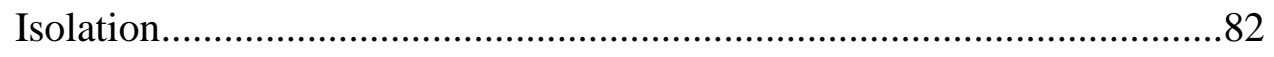

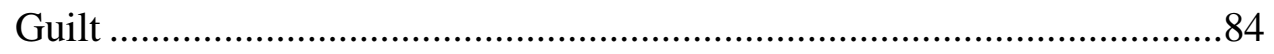

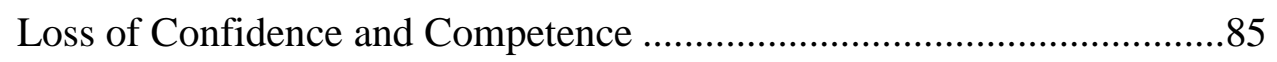

Subjective Incompetence and Demoralization.............................................90

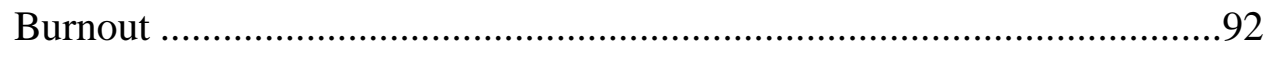

The Ending: “A Light at the End of the Tunnel”.........................................94

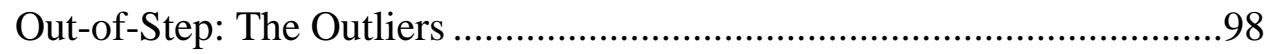


On Becoming: Assuming a New Professional Identity ..........................99

Finally . . . Accomplishment!...........................................................104

Surviving the Process: What Helped ....................................................105

Moving Out and Moving On................................................................108

Summary: Transition, Change and Transformation.............................109

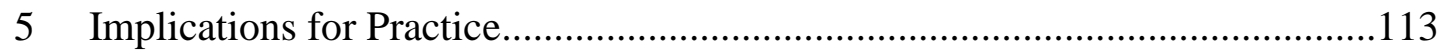

Summary of Findings.................................................................114

Trustworthiness and Rigor ...........................................................115

Strengths of This Research ..........................................................118

Limitations of This Research ............................................................119

Programmatic Interventions .........................................................121

The Role of the Cohort..............................................................121

Minimizing Competition ..........................................................123

Programmatic Reassurance ...................................................124

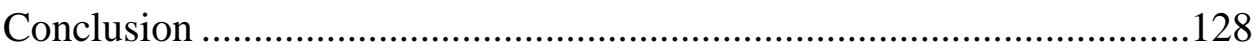

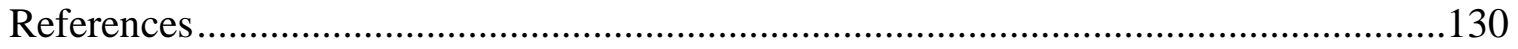

Appendices

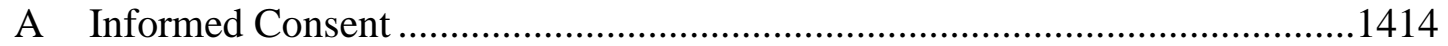

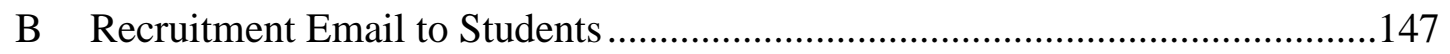

C OHSU Lay Language Protocol Summary …..................................................149

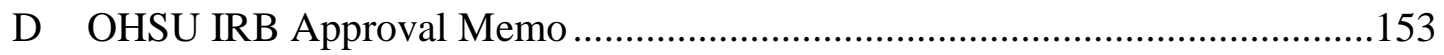

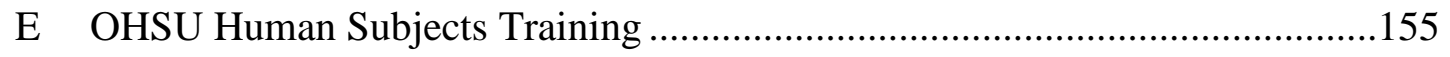




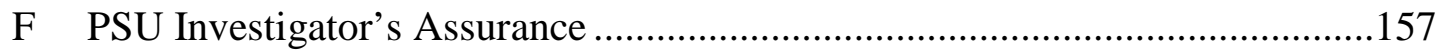

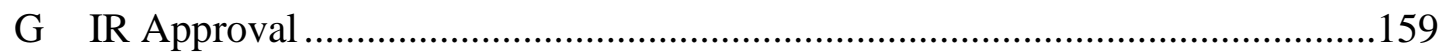

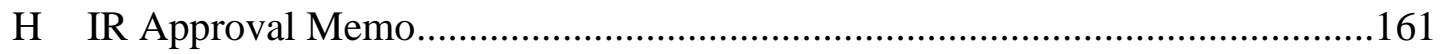

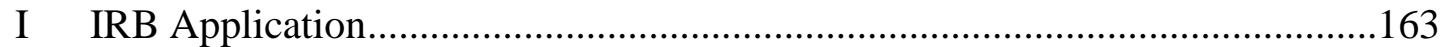

J Approved PSU Investigator’s Assurance ....................................................169

K OHSU Proposed Project Questionnaire ........................................................172 


\section{List of Tables}

Table

Page

1 Mezirow’s Transformative Learning Theory Applied to PA Education.............46 


\section{List of Figures}

Figure $\quad$ Page

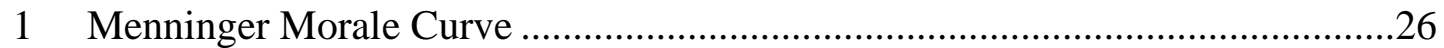

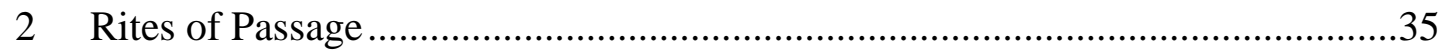

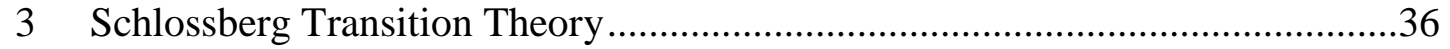

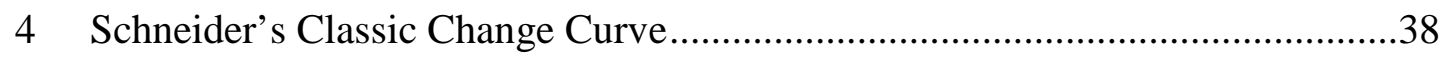

5 The U-Curve of Cross-Cultural Adaption ....................................................40

6 Phases of a First-Year Teacher's Attitude Toward Teaching............................42

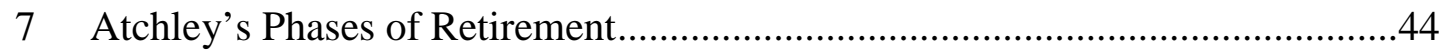

8 The Morale Curve of the Didactic Year of PA Education ...............................111 


\section{CHAPTER 1}

\section{INTRODUCTION}

Compared to the antiquity of the physician profession, the physician assistant (PA) profession is in its relative infancy and little investigation into the medical educational process and its effect on PA students has been conducted to date. A literature search reveals an initial article addressing stress and PA student adaptation to medical training in 1993 (Glicken \& Gray) in which increasing levels of stress were identified over the course of the educational process. In 2004, M. H. Brown highlighted the lack of data on the mental health of PA students and the role that PA education may play in the development of student psychiatric and psychological morbidities. Between those two dates there had not been a single additional reported inquiry into the effect of the educational process on students. Since then, a growing interest in stress during PA education has resulted in additional inquiries confirming high levels of stress among PA students (Childers, May, \& Ball, 2012; Kuhn, Kranz, Koo, Cossio, \& Lund, 2005; O’Brien, Mathieson, Leafman, \& Rice-Spearman, 2012). M. H. Brown called attention to the similarities between medical school and PA education and emphasized how the lengthier time frame allowed for completion of medical school provides additional opportunities for medical students to develop curricular mastery-time unavailable to PA students due to the condensed nature of their program, and went on to recommend the development of interventions to reduce PA student stress and the incorporation of curricular methods to increase emotional and intellectual satisfaction. Most PA programs 
do address student issues related to psychological distress on a local level, but other than stress, little additional information about the system-wide psychological ramifications associated with the process of PA education has been elaborated since M. H. Brown's note of concern. The effect of PA education on students remains speculative and anecdotal despite the growing number of students enrolling in programs nationally—an unfortunate gap in our understanding of the consequences of this medical education process experienced by these future health professionals.

As the faculty member responsible for the didactic education of the first-year class of PA students at an academic health center on the West Coast, I spend my days with highly motivated graduate students in pursuit of a professional medical career involving the provision of direct patient care. During the didactic phase of their education, each cohort of adult learners spends countless hours together going through a formalized lock-step medical education process that is rigorous, time consuming, and described by the PA profession as analogous to "drinking water out of a fire hydrant.” This metaphor graphically describes an overload of experiences and information that is beyond the capacity of students to fully absorb, yet it is this very process which has resulted in our students' successful transformation into medical professionals. Despite these successes, the workload is stressful and demoralizing at times, and student morale fluctuates accordingly. Students may be worn down by the process and may be at risk for mental and physical health challenges. One wonders if there is a way to facilitate a more humanistic approach to student learning and role socialization while simultaneously assuring the development of safe and competent health care providers. 
While acutely aware of my perception of the effects of this process on students on a daily basis, my day-to-day professional activities are not solely what sparked my initial interest in this topic; I am also seeking to understand my own personal journey as a PA student as I explore the experiences of my current students. My conceptualization of the impact that the transformative process of medical education has on the mood and morale of PA students stems from reflection on my own personal experience as a student and the ancillary effects experienced by my family and friends. As a PA student more than 25 years ago, my initial elation upon admission evaporated soon after becoming overwhelmed with the pace, quantity and complexity of the instruction. Dismay turned into despair as the year progressed. In addition to my own psychological distress, I recall the class sentiment among my cohort as increasingly negative; we felt disconnected from our faculty and we simply could not fathom what was perceived as a lack of understanding of, and empathy for, our experience. As a result, group morale fluctuated over the course of the program. Some of my classmates were emotionally sidelined by the process and experienced medical and mental health issues. In addition to managing the academic rigor of the educational process, many of us experienced crises in our personal lives, crises that we were challenged to respond to appropriately due to the rigidity of the curriculum. It was, quite simply, the most intense and relentless learning experience I have ever encountered. Although overwhelmed by the process, I ultimately evolved into a competent health care provider with new ways of thinking-clinically, professionally and personally. As a new graduate, much of the negativity about my own educational experience I attributed to personal assumptions about program weaknesses: that the challenges that I experienced were due to unintended consequences related to 
program design and not from the transformative nature of the process itself. It was not until much later-after stepping into a faculty role — that time and perspective clarity provided a more nuanced view of my student experience; transformation into a professional role is hard work and at the conclusion of the program my classmates and I were not the same people who had entered the program 2 years earlier. The program I attended provided access to a rich medical education, and I now believe that much of the dissatisfaction that I and other students experienced while engaged in the medical education process was likely a function of the difficulty of personal growth as learning and role transformation unfolds. Despite the many positive outcomes associated with socialization into a new professional role, medical education is not entirely humanistic in its design or delivery and the potential exists for students to become victims of the experience.

Having once been a PA student, my personal perspective guides my impression of the experience of PA education, but the viewpoint of a single individual cannot begin to define reality for the more than 8,000 PA students who matriculate each year in the United States (Physician Assistant Education Association [PAEA], 2015). Now that I am in the position of administering a similarly rigorous curriculum to students entering the profession behind me, I feel strongly that they deserve a thoughtful inquiry into the process and its outcomes beyond successful academic completion of the programincluding elucidation of any unintended consequences. Other than day-to-day observations by faculty across the United States, little is formally known about the lived experience of PA education. We cannot begin to understand the effects of PA education 
until the student experience of the process itself is known. In order to identify ways in which medical education programs can be supportive to students during this incredibly stressful time of personal and professional growth, more needs to be known about the process as experienced by PA students and the attendant effects of the process on mood and morale.

To examine the interaction between mood and morale within the context of what I believe to be a transformative medical education experience, I begin by clarifying the terms that form the contextual framework of issues associated with psychological states of students engaged in PA education. An overview and brief history of the PA profession will be presented, along with an overview of the typical PA program. Anecdotal observations of student mood and morale patterns will be described in order to identify the problem and illustrate the impetus for this inquiry. In chapter 2, the literature salient to the student experience is reviewed in order to provide a multidisciplinary theoretical framework from which to hypothesize the etiologies of the shared student experience. Chapter 3 outlines the qualitative methodology of the study design, fashioned to elucidate a foundational understanding of the student experience and the effect of that educational process on students. Chapter 4 describes the results of the study and dovetails a discussion connecting the data describing the student experience with the theoretical framework of this research. Chapter 5 concludes with the implications for educational practice that arose from the analysis of the study data.

\section{Definition of Terms}

Inquiry into the psychological and emotional experience of PA students engaged in a medical education process requires a shared context from which to understand the 
impact of that educational process. Before proceeding, I clarify the terms used to describe a number of constructs presented in this paper.

Mood is defined as an "an inner state that persists for some time, with a disposition to exhibit a particular emotion or affect" (Nurcombe \& Ebert, 2008, Chapter 4, Affect \& Mood, para. 2), where mood is the emotional state as described by the individual and affect is the emotional state of the individual as it appears when observed by an onlooker. Student mood varies according to circumstances in their lives, both personal and program related, however mood is considered to be more constant than the immediacy of emotion. Morale, on the other hand, refers to "the mental and emotional condition (as of enthusiasm, confidence or loyalty) of an individual or group; the level of individual psychological well-being based on such factors as a sense of purpose and confidence in the future” (Merriam-Webster, n.d.b., para. 2). The precise definition of morale is somewhat fluid; "morale is a complex of basic physical and mental states and tendencies, and of attitudes. This is the reason it is so difficult a term to define” (Sanford \& Holt, 1943, p. 93). Menninger (1975) described morale as "both an individual and collective barometer of mental health” (p. 98). It has been my observation that as each student cohort develops into a functional assemblage, a group morale also develops and fluctuates. Although morale can be referred to either positively, neutrally or negatively, the term demoralization specifically implies the loss of positive morale (de Figueiredo, 1983). As stated by de Figueiredo (1983) from the medical perspective, demoralization is "a combination of one or more observable states of distress (e.g., anxiety, sadness, discouragement, anger, resentment) with another observable state, the sense of incompetence” (p. 154). This sense of incompetence is a frequently expressed experience 
of PA students who find their old competencies no longer valued at a time when they have yet to develop new ones. The construct of demoralization has been only superficially mentioned in medical education, so little is known about its impact in an educational setting. It has, however, been identified in the psychiatric literature as a possible precursor to depression (de Figueiredo, 1993). Demoralization so clearly describes what students subjectively report that I use it here to imply loss of positive morale accompanied by the sense of subjective incompetence. Psychological distress is a nonspecific term applied to a variety of “negative mental health states” (Veit \& Ware, 1983, p. 731) - including stress, anxiety, sadness, frustration and depression. These negative mood states occur on a continuum from mild to severe and can occur singly or in combination. When psychological distress reaches a certain threshold, a psychiatric diagnosis may be made. Psychological well-being, or a state of positive mental health, is described as "feeling cheerful, happiness, satisfaction and enjoyment of life” (Veit \& Ware, 1983, p. 730). Good or intact mental health implies the absence of a diagnosable mental disorder as well as having psychological well-being and the positive attributes of competency and flexibility in adapting to interpersonal relationships and life's challenges. Feeling comfortable with oneself, ones circumstances and with others is a key component of having intact mental health. Finally, the term cohort is described as "the aggregate of individuals (within some population definition) who experienced the same event within the same time interval” (Ryder, 1965, p. 845). Cohort data, according to Ryder (1965) are “ordinarily assembled sequentially from observations of the time of the occurrence of the behavior being studied, and the interval since occurrence of the cohort- 
defining event” (p. 845). I use the term cohort to denote our annual group of PA students who matriculate into an entering class, simultaneously experience a lock-step curriculum involving socialization into a new role and who ultimately complete the program together as a group before moving into their professional roles as healthcare providers.

In addition to defining terms, it is important to also define the concept of the PA and their role in medicine. As a relative newcomer among health care professions, confusion about PAs and their scope of practice does exist, particularly in light of the term "assistant" in the professional title. In the next section I discuss the role of the PA and describe the historical context surrounding the origination and development of the profession, after which I provide an overview of the process of PA education.

\section{Overview of the PA Profession}

PAs are highly trained medical professionals who have completed an intensive medical education designed to prepare them to provide a variety of medical services. The American Academy of Physician Assistants (AAPA, 2014) has defined PAs as:

A medical provider who is nationally certified and state licensed to practice medicine (AAPA Fact Sheet, para. 1). PAs can obtain medical histories, conduct physical exams, diagnose and treat illnesses, order and interpret tests, perform medical procedures like joint injections, counsel patients on preventive healthcare, assist in surgery, write prescriptions, and make rounds in nursing homes and hospitals, among many other medical services. (AAPA Fact Sheet, para. 3)

As part of a physician-directed team, PAs have been shown to provide quality of care comparable to that of a physician (Office of Technology Assessment, 1986; Sox, 1979) while improving the cost effectiveness of a medical practice (Medical Group Management Association, 1996). Additionally, PAs have been shown to be able to care for most patient problems seen in the primary care context (Hooker \& Cawley, 2003; 
Office of Technology Assessment, 1986; Record, McCally, \& Schweitzer, 1980), are utilized clinically in nearly all medical and surgical subspecialties, and are increasingly being utilized to fill provider gaps due to physician shortages (Glicken \& Miller, 2013).

The PA profession is still a relatively new addition to the health care arena.

During the 1960s a number of factors converged, culminating in the development of the PA profession (Cawley, 2007; Cawley, Cawthon, \& Hooker, 2012). Innovations in medical education were being explored by organized medicine and the federal government as a means of expanding and redesigning traditional medical curricula during a period of nationwide physician shortages (Ludmerer, 1999). Coincident to this, servicemen with significant Viet Nam battlefield experience as medical corpsmen were returning to the United States upon the conclusion of their military service unable to find a civilian outlet for their skills (Ballweg, Sullivan, Brown, \& Vetrosky, 2008). Physicians at Duke University sought to capitalize on the experiential skill set of this returning pool of servicemen and created the inaugural class of four PA students in 1965 (Cawley, 2007). The original PA training was fashioned after the WWII fast-track physician training model designed to get new physicians through their medical education process in an abbreviated amount of time during a period of wartime need (Ballweg et al., 2008). Since its inception at Duke, this fledgling medical profession has grown to include more than 100,000 certified PAs in clinical practice in the United States (AAPA, 2014). Data reported in 2015 identified approximately 19,000 PA students enrolled in PA programs in the United States (PAEA, 2015); programs are now being started in countries around the world (Ballweg et al., 2008). Originally an all-male profession, women now comprise two thirds of the PA student population in the United States (Cawley, 2007). 
As the profession has matured and become well accepted, the demand for PA services has increased. The Bureau of Labor Statistics (2015) consistently ranks the PA job market as one of the 30 fastest-growing occupations in the country and projected 30\% employment increase expected during the 10-year period between 2014 and 2024. U.S. News \& World Report (2015) lists the PA profession in the top 10 of the 100 best jobs in the United States and reported excellent future job opportunities that are expected to continue through the next decade. Health care reform and the improved access to care among currently and formerly uninsured Americans is dramatically increasing the demand for practicing PAs able to provide care to this expanding patient population (AAPA, 2010, 2011; Patient Care and Affordable Care Act, 2010). The positive job outlook combined with the appeal of a shortened (and consequently less expensive) medical education process has fueled the development of a large pool of prospective applicants. During the 2013-2014 application cycle, our academic health center received more than 1,000 applications for our class of 38-42 seats, resulting in an increasingly competitive admissions process. As the pool of potential students has expanded, the number of training programs has also grown. Currently, there are more than 190 programs in the United States, with approximately 7,000 new PAs graduating annually (AAPA, 2014).

Although each PA education program in the United States has its own unique style and format by which the educational model is delivered, accreditation requirements set the standards for structure and content of the educational process (Accreditation Review Commission on Education for the Physician Assistant, 2010). Applicants satisfy typical prerequisite requirements that include a baccalaureate degree, completion of a 
number of basic science courses and patient care experience in a health care setting. Once successfully admitted, students participate in a rigorous and intensive full time educational immersion process lasting an average of 26 months (AAPA, 2010). The first year is primarily didactic in nature and consists of basic science, clinical medicine and behavioral medicine content. Class work during the didactic phase is quite different than the typical undergraduate or graduate course schedule in that it generally consumes the majority of each weekday. The average PA student is in class from eight AM to five PM on most days; a relentless schedule that often precludes studying during the daytime hours and frequently interferes with family life.

The second year clinical phase of PA education consists of structured apprenticestyle experiential rotations during which students directly participate in the supervised care of patients in a variety of settings and specialties including inpatient medicine, primary care/ambulatory medicine, orthopedics, general surgery, obstetrics/gynecology, emergency medicine, and pediatrics. For our students, this phase represents a nomadic year as rotations occur throughout the Pacific Northwest; moving every 5 weeks to a new clinical site is the norm. Regardless of the physical location of rotations, these clinical experiences provide students with more than 2,000 hours of directly-supervised patient care prior to graduation. The PA educational process is frequently described as constituting "seventy-five percent of medical school in fifty percent of the time” (Ballweg et al., 2008, p. 46) and prepares PAs to perform approximately $80 \%$ of medical services that are traditionally performed by physicians (Ballweg et al., 2008).

Graduates of accredited PA programs must pass a national certifying examination to be able to call themselves "Physician Assistant—Certified" or "PA—C" (National 
Commission on the Certification of Physician Assistants, 2014). To maintain this certification, PAs must complete 100 hours of continuing medical education every 2 years, and successfully complete a comprehensive recertification examination every 10 years (National Commission on the Certification of Physician Assistants, 2014). Once certified, PAs obtain licensure to practice medicine through each state's regulatory agency, typically a Board of Medicine.

\section{Anecdotal Observations of Patterns of Student Morale}

Inherent to the success of our program is the "raw material" with whom we are privileged to work - the students themselves. Selected from a large and competitive applicant pool and essentially strangers to each other when they arrive on campus, our matriculating students quickly develop a group identity as a cohort and seem to emulate certain patterns of individual and group behaviors that appear to be repeated from year to year. Faculty from within our program as well as from other programs around the United States have commented anecdotally on what appears to be a cyclic pattern of class morale as students engage this challenging process, yet no formal research has been conducted on this phenomenon to date.

This seemingly predictable pattern of observed cohort mood and morale begins at matriculation, where having survived a competitive and rigorous application process for a limited number of class seats, new students arrive on campus elated and motivated to begin the process of becoming a PA. Restless during orientation activities, they seem impatient to "get on with the process" and begin their coursework; their mood is positive and hopeful. Campus welcoming activities provide students' families and friends with an opportunity to publically celebrate their loved ones’ admissions success; familial pride is 
clearly evident. Once formal courses begin and syllabi are distributed, student excitement is tempered by increasing anxiety about the ever-expanding workload while juggling organizational tasks and getting acquainted with faculty and fellow cohort members. Faculty report a significant amount of student confusion and frustration during this early phase as students acclimate to the academic environment and begin to conceptualize expectations regarding course requirements. By the time students have been engaged in classes for a few weeks the reality of the workload is fully revealed, their elation at admission has waned and they begin to appear overwhelmed. Many of our students have never experienced a full-time educational process requiring participation in day-long classroom activities up to five days a week, and they find the adjustment to be a significant challenge. Additionally, the emphasis by graduate medical education programs on the development of self-directed learning skills and student-focused discovery represents a rude awakening for some students who hail from undergraduate environments where concrete learning modalities and defined expectations such as assigned readings were the norm. Along with adjusting to this unfamiliar educational environment and the rigorous curricular material, students also undergo significant upheaval in their sense of themselves. Whereas they may have been highly functioning individuals with finely honed skills and well developed career identities prior to becoming PA students, they are now all novices in a new profession. The transition from “competent” to the “incompetent” role (Daley, 1999; Terry, Callan, \& Sartori, 1996) creates significant discomfort for many students and likely contributes to the development of psychological distress affecting cohort morale. 
From that initial elation at matriculation where the entire cohort revels in esprit de corps, group morale is observed to gradually erode as the realities of the workload continue to unfold. After being in class most of the day, students must balance the need to study with the demands of their personal lives. Family members initially so supportive of their loved one's entrance into this educational process may come to resent how little they are able to see their student; even when at home, the student is often distracted by academic responsibilities. Friends and relatives describe feeling abandoned and unimportant as personal interactions take a back seat to educational demands. Conflicts with personal relationships seem to increase as the workload increases, and by the midpoint of the didactic year cohort morale appears to sink to a new low. Students report fatigue, demoralization, anxiety and frustration. They complain that they will never be able to learn everything necessary to be a safe and effective medical provider, and they doubt their ability to retain the vast amounts of material they have been exposed to during the previous months. Though steadfast in their goal of completing the program and becoming PAs, the relentless nature of the educational process wears them down and the mood of the group becomes increasingly subdued. At times, the sense of demoralization and despondency among the cohort is unmistakable.

As the year progresses and the end of the didactic chapter of their training approaches, cohort morale seems to turn the corner and a gradual lightening of mood is noted by students and faculty alike. Students express astonishment that the end of the classroom phase of their education is in sight and energy seems to be enhanced. Comments about the "light at the end of the tunnel" no longer refer to oncoming trains or other disasters, but of achieving their goal of surviving the academic year. Their inquiry 
skills and self-directed learning abilities have matured significantly, and they are increasingly competent at defining clinical questions and locating empiric, evidencebased information with which to drive their thought processes and decision-making. As they improve their ability to distinguish relevant material from that which is irrelevant, student efficiency in studying improves, resulting in a reduction in time necessary to complete academic tasks. Plans for the upcoming clinical phase of the program serve as a powerful motivator for students to redouble their efforts toward continuing their educational quest. Yet as the end of the didactic year approaches, excitement about the next phase of the medical education process is blunted by increasing anxiety; many students harbor deep reservations about their readiness to leave the familiar classroom for the-as yet-mysterious clinical phase. They doubt the depth of their acquired medical knowledge and worry that learning accomplished during the didactic phase has not adequately translated into retained and retrievable memories. This concern coalesces into the fear that they are unprepared and unqualified to tackle the challenges of the clinical setting, a fear that is not resolved until after beginning the next phase of their education. The end of the didactic year, then, is characterized by improved mood and excitement tempered by anxiety and self-doubt.

\section{Significance of This Project}

It is this pattern of mood and morale that seems to recur as each new PA student cohort matriculates into the program. As the primary faculty member overseeing the flow of the didactic year, I personally witness this phenomenon annually. The regularity by which this observed pattern is noted, both by myself, fellow faculty members at our 
academic health center and by faculty at other programs around the United States, suggests the presence of a predictable developmental process, yet little is formally known about the experience of PA students as no prior investigation has been undertaken. Is there value in exploring this phenomenon?

Beyond simply satisfying faculty curiosity regarding student mood and morale in order to confirm or refute the presence of a predictable pattern, there are several compelling reasons why investigation into this phenomenon is warranted. First, there is ample evidence that graduate medical education causes increases in psychological distress (including stress and anxiety) among medical students (Finkelstein, Brownstein, Scott, \& Lan, 2007; Henning, Ey, \& Shaw, 1998; Schmitter, Liedl, Beck, \& Rammelsberg, 2008; Vitaliano, 1988; Vitaliano, Russo, Carr, \& Heerwagen, 1984), yet there remains a limited amount of empirical evidence about the effects of this process on PA students (M. H. Brown, 2004; Childers et al., 2012; Glicken \& Gray, 1993; Kuhn et al., 2005; O’Brien et al., 2012). Inquiry into whether similar outcomes exist among PA students would improve the understanding of educational practices among PA educators and provide a foundation on which to base informed curricular decisions.

Additionally, stress and anxiety have both been implicated as major causes of cognitive dysfunction. While some stress is conducive to learning (McGrath, 1976; Vitaliano, 1988), excessive levels have been shown to impair learning (Schwabe \& Wolf, 2009). This hindrance to learning has the potential to impact the quality of future patient care should students miss critical content knowledge due to psychological distractions. Programmatic overload of students while simultaneously limiting time for the personal renewal necessary for the maintenance of mental health may actually impede rather than 
foster student learning (Wolf \& Kissling, 1984), an outcome contrary to programmatic educational goals. Attention to stress levels inherently ingrained in the medical education process can improve student outcomes (Coombs, Perrell, \& Ruckh, 1990).

Another effect of stress and demoralization during a rigorous medical education is the loss of empathy, well described among medical students (Bellini, Baime, \& Shea 2002; Dyrbye et al., 2010; Hojat, Mangione, \& Nasca, 2004; Krasner et al., 2009; Pederson, 2010; Rosenthal et al., 2011; Wear, Aultman, Varley, \& Zarconi, 2006; Woloschuk, Harasym, \& Temple, 2004). The trajectory of PA student empathy during their educational journey demonstrates a similar decline as those of their medical school colleagues (Mandel \& Schweinle, 2012.) The ability to be empathetic with patients who are injured or ill is a critical component of appropriate and compassionate patient care and a key construct of the medical practice of PAs. Coombs et al. (1990) described healthy professional development as that which includes attention to not only the head (knowledge) and the hands (skills), but of the heart (attitudes) as well. If the educational process itself contributes to impairment of students' ability to maintain their empathetic engagement with patients, identification and rectification of those impairments is not only in the interest of improved patient care but in the interest of the enhanced professional development of our future PAs as well.

In addition to the potential impact on patient care, we must further consider the impact of the educational process on students themselves. Medical and dental students are known to have higher incidences of mental health and substance use issues than the general population (Cardall, Rowan, \& Bay, 2008; Dyrbye et al., 2008); less is known about the mental health of PA students (M H. Brown, 2004; Childers et al., 2012; Glicken 
\& Gray, 1993; Kuhn et al., 2005; O’Brien et al., 2012). Although there have been reported suicides among PA students nationally, there are no formal data on suicidality nor of circumstances surrounding student mental health. If a predictable pattern of cohort morale exists, and if the period(s) of low cohort morale corresponds with student demoralization and psychological distress, the potential exists for PA program administration and faculty to implement effective interventions. For example, stress reduction through the teaching of coping and time management skills has been found to facilitate student resilience during medical school (Coombs et al., 1990); similar interventions might be therapeutic among PA students during their rigorous academic experience. As medical educators we need to facilitate the transformational growth and professional development of PA students by recognizing patterns, clarifying experiences and identifying opportunities to utilize interventions designed to enhance the student experience while also ensuring the humane and effective education and socialization of our future health care providers. According to Coombs et al. (1990), “most medical schools do little to meet the emotional needs of trainees until after they create academic and emotional problems that require obvious attention” (p. 579). Given the absence of information about the PA student experience, inquiry into this perceived pattern of mood and morale holds the potential for improving the PA profession’s grasp of medical education practice, its effect on student outcomes and its potential for impact on the future care of patients.

\section{Research Questions to be Addressed}

Due to the limited amount of information regarding student experiences while engaged in PA education, there are a number of inquiries that could provide guidance for 
future enhancement of the educational process and its effect on students. What is the PA student's experience of the educational process? In what ways do these experiences affect students? And, how do those experiences affect student mood and morale?

Investigation into perceived fluctuations in morale would provide an answer to the questions: What are the critical themes regarding the process of PA education and its effects on students? Is there evidence to support the existence of a recurring pattern of morale among student cohorts during the didactic phase of PA education? As a cohort consists of individuals who take on a group identity, it would be important to further distinguish group morale from individual mood and determine if there is evidence of psychological distress among PA students during the didactic phase. And, if distress is identified, in what way does it correspond to periods of high and low cohort morale? If a predictable pattern of morale over time is identified, programs could conceptualize psychological and curricular interventions to ameliorate psychiatric or psychological symptomatology while simultaneously maintaining the high standards of academic rigor and excellence necessary to develop health care providers capable of delivering high quality, safe and effective medical care.

In addition to enhancing the PA student's experience and the PA educators' understanding of the practice of PA education, it is my belief that investigation into these questions can also highlight important theoretical connections not previously described in PA education. In particular, is there a relationship between the theoretical models of change theory, transition theory, transformative learning and cross-cultural adaptation and the patterns of mood and morale exhibited by students while undergoing the developmental process to becoming a health care provider? In the next section, the 
literature regarding mood and morale is presented along with a discussion of identity formation and professional development, In addition, the theories addressing the process of human change are described as potential explanations for some of the challenges inherent to the medical education process. 


\section{CHAPTER 2}

\section{REVIEW OF THE LITERATURE}

The PA profession frequently describes the process to become a PA as similar to drinking water out of a fire hydrant; a vivid metaphor for the experience of participating in a nonstop, demanding and rigorous medical education. Although PA educators witness the effects of this process on PA students with each entering cohort, it is an area of inquiry that has previously gone unexamined. To date, little is formally known about the student experience beyond the personal experiences of faculty during their own education or through their day-to-day engagement with PA students. Although much has been written about the success of the profession generally (Ballweg et al., 2008) and its effectiveness in developing proficient health care providers (Medical Group Management Association, 1996), to date there has been no formal attempt to understand the student experience of this process. Observed fluctuations in student mood and morale clearly suggest that something is going on worthy of investigation and elucidation. The following literature review will examine several multi-disciplinary theoretical models that may help to describe the underpinnings of the student experience. In particular, the literature on change theory, transition theory and cross-cultural adjustment may shed light on the process of becoming a health care provider, as could the theory of transformative learning. To begin the discussion, however, I would like to first introduce the article that sparked my earliest interest in the behavioral phenomenon that I observe in my students. 
Not long after assuming my first PA faculty role in 1994, I was given a muchphotocopied and dog-eared essay written by Seashore (1975), entitled “In Grave Danger of Growing: Observation on the Process of Professional Development.” It served as a noteworthy description of my own personal experience as a student and validated my faculty observations of class dynamics as well. Seashore's words created a personal “aha” experience and permanently altered my perspective on the experience of a transformative educational process. The assertions he made have remained with me throughout my career as a medical educator, and I consider his article to be a seminal and timeless description of morale among students participating in a professional medical education process. Seashore, a social psychologist, postulated the presence of predictable patterns of mood and morale in professional development programs, particularly focusing on those with cohorts of students involved in lock-step curricula. He began his discussion by describing what he called "strange goings on” (p. 1) among students undertaking these programs, including:

- Most students will experience levels of stress and anxiety that effectively block any significant learning during phases of the program regarded as critical by the faculty.

- A fair number of persons who have been functioning quite competently in work, family and social settings will experience periods of being deskilled, incompetent, emotionally out of commission, or just plain confused after entering the program.

- Learning how to better oneself in the helping process is likely to change one's basis for self-esteem, and alter what it is one values in oneself or others. This produces a significant amount of conflict among those who liked you for what you were, not for what you are becoming.

- Students report feelings of anger, depression, resentment, envy and irrelevance among family and close friends because of their participation in the program, 
- Participants often report that they are investing at least several times more energy in the program than they expected to. (p. 1)

Experiences shared among my faculty colleagues also support Seashore’s observations, and it seems that individuals engaged in rigorous medical education programs may share similar experiential challenges during their training regardless of where or when that training might have occurred.

Seashore (1975) wondered what it is about these types of programs that creates such significant emotional reactions among students, faculty, family and friends. He proposed several reasons for the challenges experienced during professional development programs — one of which stands out as potentially pertinent to the experience of PA students. He described a "mini-life cycle" (p. 2) as a central feature of intense programs suggesting the presence of a predictable pattern, including typical changes in group morale over time. “As if guided by unreachable and unaccountable outside forces, the program may also induce a natural cycle affecting the morale of its members simply because it has a beginning, middle and end” (p. 2).

The foundation for this program mini-life cycle as postulated by Seashore is based on research done on early Peace Corp volunteers (Menninger, 1975), where morale was measured over the course of participants' 2-year field commitments. Out of this research, the Menninger (1975) Morale Curve, a graphic representation of group psychological experiences, was developed. On arrival at their new Peace Corps assignments, Menninger observed group enthusiasm and excitement-happy to finally be engaged in their new roles, yet anxious about the unknown and their ability to succeed. Motivation was high, yet apprehension was also prominent as the volunteers anticipated their unknown futures. 
This entry phase was titled the Crisis of Arrival, and was described as

phenomenologically similar to "culture shock”- “an anxiety that results from losing all one’s familiar cues” (Menninger, 1988, p. 207). L. Brown and Brown (2009) similarly identify the emotional reaction that participants experience when removed from their familiar environs as culture shock, and further describe it as chaos.

Menninger’s (1988) Crisis of Arrival, initially characterized by high morale, was soon followed by a downturn in morale as participants struggled to orient to their new roles. They were challenged with the reality of loss-loss of established relationships, familiar routines and a diminished sense of competence. This loss of mastery may exemplify “subjective incompetence” (de Figueiredo, 1983, 1993), a feature of demoralization later described in the psychiatric literature (Clark \& Kissane, 2002) that may further elaborate the realization of losses that take place during the crisis of engagement. Bandura (1982) described the sense of futility individuals may develop when they perceive themselves to be ineffectual, leading to "self-devaluation and despondency” (p. 140), a condition not unlike subjective incompetence. During this time of initial adjustment, the Peace Corps volunteers also recognized that the expectations they brought to their commitment differed from their actual experience; their romanticized vision of what their service would entail gave way to the less rosy reality of the situation. Morale during this phase tended to drop significantly, leading to the Crisis of Engagement. Menninger explained,

with regard to lost expectations, the degree of depression or drop in morale is a function of the disparity between reality and expectations for the new situation. The closer the new life situation to what was planned for and anticipated, the less the experienced "loss." (p. 208) 
The next phase, Crisis of Acceptance, found the participants coming to grips with their new reality and they learned how to assess their situation with a more realistic eye, resulting in improved morale. Participants were described as achieving "a new sense of self” (Menninger, 1988, p. 208) with improved mastery over their new life circumstances.

Finally, as the end of the program approached, participants faced the Crisis of Re-Entry and another fluctuation in morale. Although excited about the upcoming changes associated with the culmination of their volunteer service and looking forward to returning home, they now experienced mixed feelings about leaving the program they had acclimated to and regretted saying good-bye to fellow participants. The transition associated with departure from the program resulted in a reactivation of the anxiety associated with the Crisis of Arrival regarding the unknown aspects of the next phase in their lives (see Figure 1).

Seashore (1975) saw utility in applying Menninger’s morale curve to the family medicine residency program he was working with as it illustrated similar patterns he had observed in the medical education of physician residents-periodic changes in group morale based on crises experienced during the process of professional development. Is this process what is observed with each of the student cohorts enrolled in the PA education process? The pattern of morale observed each academic year mirrors this phenomenon, beginning with the excitement and nervousness of the new matriculants who arrive on campus after expending great effort to secure a seat in the class, soon followed by the declining mood of the now too-busy students who have come to realize their immense workload as the fall and winter terms approach. The relentless nature of 
the program is in full swing during this time, and students encounter nonstop delivery of content material followed by examination after examination. In the spring an upswing in mood is noted as students begin to develop improved competence and confidence and they begin to see the light at the end of the tunnel—a hopeful sign that they will succeed after all. Finally, as the end of the didactic year approaches, increasing anxiety and apprehension becomes apparent as students are faced with the unknowns of the next phase as they move into the clinical training setting.

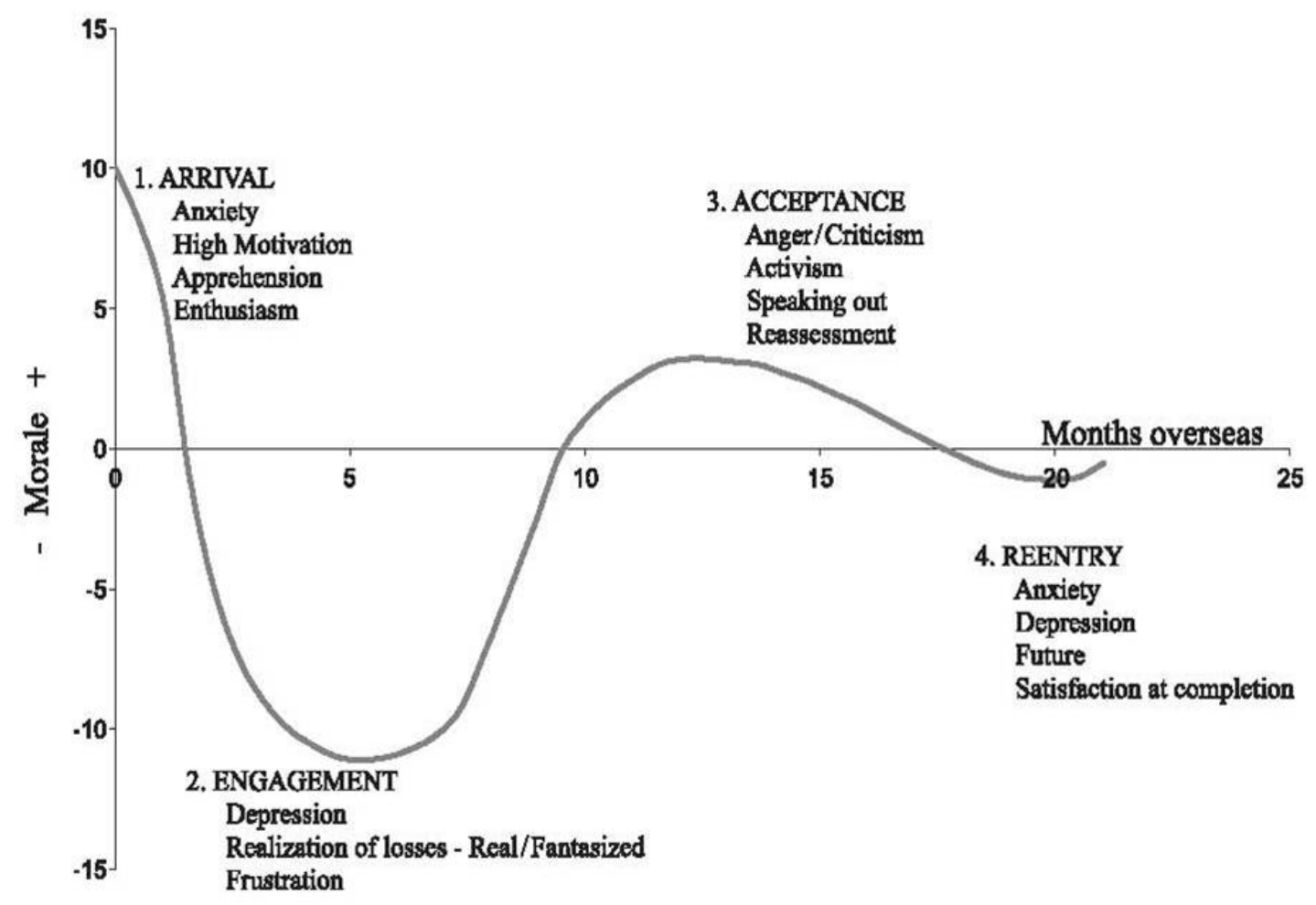

Figure 1. Menninger morale curve. Source: Menninger (1975).

Menninger $(1975,1988)$ speculated that his morale curve pattern had the potential to repeat according to the structure of the social circumstance; in academic terms, participants could theoretically experience this morale curve over the course of an entire 
program or its subparts - a year, a term or even a workshop. "The curve is extensible or contractible; it can be useful in interpreting student—and faculty-ups and downs during the college year . ..” (Menninger, 1975, p. 110). As a result of repeated observations of student cohorts over a number of years, this phenomenon may be occurring predictably during the didactic phase of PA education.

Further exploration of the construct of morale is worthwhile in order to elucidate the ways in which an educational process can affect morale. In the United States during the Second World War, discussions about morale included references to both civilian and military morale (Stafford-Clark, 1949). Menninger’s (1975) research on the experience of Peace Corp volunteers was based on studies of wartime morale among World War II servicemen who were asked questions such as "what were the critical points in a tour of duty? ... what could we do to limit the 'casualties' (of the experience)? . . . what support could we give that would make a difference? [and] . . . could we predict certain kinds of reactions (from participants)?” (p. 101). Answers to these questions culminated in the description of morale as the predictable response to a change in life situation. As participation in a professional education program certainly qualifies as a change in life situation, these questions seem every bit as valid to ask of health professions students today, and attention to the answers could ostensibly guide programmatic design.

The components necessary for the maintenance of positive morale have been postulated to include a functional support system, a grounded perspective where the individual learns from the past but focuses on the future, a realistic outlook (neither optimistic nor pessimistic) and the ability to assume personal responsibility for one’s 
actions (Richards, 1999). Each of these components can be disrupted by the process of change experienced at the initiation of a rigorous educational program; loss of any of these components could presumably to impact morale. Menninger (1975) believed that when life circumstances change, an individual's “sense of himself undergoes changes that produce the stresses reflected by the morale curve” (p. 110). The construct of change, then, is an important mediator for morale, and the morale curve may be the graphic representation of the human experience of transformative change in which morale is a barometer for the stressors inherent in the transition itself. Are the observed shifts in student cohort morale a function of transformative change? Although numerous studies show episodic change in mood and morale at given points within health professions curricula (Bellini et al., 2002; Cardall et al., 2008; Finkelstein et al., 2007; Spiegel, Smolen, \& Jonas, 1986), little data exists on student morale on a continuum over time, and connections between transformative change and student morale are tenuous at best. Yet it seems likely that a relationship exists even if not yet well delineated. Further, there does not appear to have been any exploration of the student perspective nor their experience during a professional development process, and there is little acknowledgement of the relationship of the educational experience with student mood and morale. Before investigating the concepts of transformation and change, however, a discussion of identity development is warranted. Just as Peace Corps volunteers experience a dramatic shift in their identity when beginning their service (Menninger, 1975), so, it appears, do newly matriculated PA students. The next section provides an overview of identity and professional development to explore the foundation for the transformative medical education experience. 


\section{Identity and Professional Development}

A significant task required of all health professions students is to undergo transformation into a new professional role and identity. Erikson $(1956,1980)$ is credited with the concept of identity as a psychosocial process wherein the individual coalesces interactions of physical and cognitive growth with the requirements of his or her environment. The emergence of identity as an evolutionary progression through which the self is continually revised suggests a constructive-developmental perspective of personal growth by which the individual builds new meaning upon past understanding. Erikson (1980) described identity as "a gradual unfolding of the personality through phase-specific psychosocial crises” (p. 128), and “each successive step . . . is a potential crisis because of a radical change in perspective” (1956, p. 57). This reference to crises and perspective change reflects an important connection to Mezirow’s (2000) transformative learning theory which is discussed later. Before doing so, however, it is worth elaborating on the concept of identity further as it is viewed differently through the lens of the psychologist versus that of the social scientist. Many psychologists conceptualize self and identity as:

(a) psychological contents such as self-categorizations, beliefs, and goals,

(b) psychological processes associated with awareness such as self-appraisal, self-definition and self-reflection; and (c) the personal project of self-development in which individuals construct a representational self-system. (Roeser, Peck, \& Nasir, 2006, p. 391)

Social scientists, on the other hand, view self and identity as:

(a) social and cultural structures that assign to individuals various consequential group labels (social categorizations), statuses and roles, (b) interpersonal processes associated with the appraisals of, and social positioning by, other people; and (c) the sociocultural project of self-envelopment by which individuals, based on their array of group memberships, develop multiple 
identities through patterns of participation in shared activities. (Roeser et al., 2006, p. 392)

From a simplistic perspective then, these points of view from two disciplines describe a distinction between the concept of self-identity as development of individual psychological factors and the concept of role identity as role development that is socially constructed. PA education involves psychological development of identity as students experience personal growth through the educational process of learning medicine. In addition, student development of a professional identity as a health care provider is achieved through the process of socialization into a role possessing a set of characteristic behaviors and expectations (Biddle, 1979). Given that students must be taught what it means to be a PA, the socialization process is the method through which they apply the acquired language and content knowledge to the skills and behaviors common to the role of PA. Biddle (1979) described this socialization process as assimilation, where the individual and the social system interact. The resulting accommodation, adaptation and adjustment of the individual is said to be "reached when the person is no longer distinct from the other members of the social system into which he or she is passing” (p. 288). In order for students to adopt professional norms and assure their incorporation into the professional role of PA, they must first engage an organizational socialization process through the educational system (Van Maanen \& Schein, 1979), and student success is predicated on active participation in their own socialization process (Gardner \& Barnes, 2007).

The psychological and sociological development of self and identity is only part of the outcome of an intensive medical education experience, but one in which the 
individual's sense of themselves is altered or transformed. The next section discusses the concepts of transformation, learning and the change process in order to postulate underlying reasons for fluctuations in student morale.

\section{Transformation, Transition and the Change Process}

The themes of transformation, transition and change, where individuals move from one role or point of view to another, are descriptive of cohorts who experience dramatic shifts in their lives as a result of undergoing a significant change process (Marks, 2007; Terry et al., 1996). Bridges (1985) distinguished change from transition by describing change as something that can be foreseen and planned for that is associated with the starting or stopping of some activity. Moving an appointment time then can be seen as a change. A transition, however, is an "inner reorientation process that individuals and teams go through when some change requires them to stop doing things the way they used to and start doing them differently” (Bridges, 1985, p. 30). PA students appear to undergo profound change as they transition into the clinician role through their transformational educational journey; their perspectives are different by the time they reach the end of the program having developed sophisticated new ways of thinking and problem solving. Is the process of transformation expressed through the emotional barometer of morale as suggested by Menninger? Are we observing the transformation of students through their emotional experience of the PA education process?

Lewin (as cited in Deutsch, 1954), credited as the founder of the social psychology movement, developed a framework for analyzing the driving and restraining forces that affect the stability of human behavior. Lewin’s (1947) “force-field analysis” laid the foundation for one of the earliest models to break down the components of 
human change, a three-stage process involving unfreezing-change-freezing. Unfreezing is the process by which an individual prepares to undergo a change, including surmounting the forces that encourage inertia. Unfreezing requires overcoming ambivalence about change and enhancing motivation to undertake the process ahead. Dissatisfaction with one's life path is a common motivator for prospective PA students seeking admission to a PA training program, a course of action requiring them to unfreeze. Many individuals seek to enhance their role in helping patients once they realize their impotence to do so without the acquisition of additional skills and training. Once unfrozen, change takes place. Lewin (1947) identified the change portion of the process as the primary source of psychological distress due to discomfort and fear about the unknowns ahead. Change is not without emotional challenge, including a sense of loss for what is left behind (Bridges, 2003; Kubler-Ross, 1969). Once through the change itself, Lewin (1947) postulated that individuals experienced a freezing process as they adapted to the outcome of the change by acclimating to the new state of normality. This unfreeze-change-freeze model could describe the process of PA education as students arrive dissatisfied with their old roles ready to take on the challenge of a new profession. They are soon met with the unsettling prospect of giving up their old ways of thinking in order to assume new competencies as a PA (unfreezing). The next interim phase of change creates ambivalence and anxiety as students experience loss of their old identity before assuming their new one (change), an emotionally challenging process accompanied by a downturn in morale. Finally, as their knowledge base and skills develop, confidence in their new role solidifies. Whereas once undifferentiated, they are now shaped into a new professional identity (freezing). Once socialized into the role of a provider, an individual 
can never return to their former unknowing state; thinking as a clinician is not something that can be unlearned. "As the old adage goes, you cannot simply add the notion that the world is round to the notion that the world is flat. Some types of learning clearly have this transformative effect ...” (Robertson, 1997, p. 109).

Another perspective on transformation is provided by Bridges (2003), who described phases of change in a slightly different way. His model of transitions, like Lewin’s (1947) similarly includes a three-part process that takes place when individuals are confronted with change. Bridges' “Ending, Losing, Letting Go” (p. 7) phase suggests that for something new to begin, something else must end. Even if this represents a desired change, such as matriculation into a much anticipated educational program, there remains an element of bereavement associated with this first phase of letting go of old ways in anticipation of new horizons. When loss occurs, people often respond in emotional ways, including symptoms of depression, sadness, fright and confusion, all signs of grieving (Kubler-Ross, 1969). Anatole France (1844-1924), a French poet and novelist is credited with saying, “All changes, even the most longed for, have their melancholy; for what we leave behind is part of ourselves; we must die to one life before we can enter another” (Marks, 2007, p. 724). Resistance to change then "is not necessarily that of resisting the change per se but is more accurately a resistance to losing something of value to the person” (Marks, 2007, p. 724). In this case, the loss is that of their former identity. After confronting the challenge of letting go, the next phase encountered in Bridges' model is the "Neutral Zone” (p. 8) in which the old ways of being are not completely gone, yet the new ways have not been fully assimilated. Bridges characterized this as a psychological “no-man’s land” (p. 8) between the old and new 
identity, fraught with discomfort and confusion and ultimately where transformation occurs. Although unsettling, "it is the chaos into which the old form dissolves and from which the new form emerges” (Bridges, 2003, p. 9). Ultimately, according to Bridges, individuals emerge from the "Neutral Zone” into the "New Beginning” (p. 57) where a new sense of identity is developed. At this point, the individuals are ready to move forward in their new role with improved clarity and confidence.

Although originally used to describe the workplace, I believe Bridges’ (2003) model could also be applicable to the educational setting as it represents a constructivist model of new and transformational experiences common to medical education. Our PA students matriculate with educational capital, life skills and patient care experience. Shortly after arrival, they quickly lose their former identities of nurse, paramedic, pharmacy technician, etc., and become undifferentiated novices. Confidence is shaken when formerly competent adults are returned to the beginner role. After a period of time letting go of these former roles, students spend months in a confused state as they learn a huge body of knowledge while also undergoing a socialization process to become a new type of health care provider. Finally, they emerge with a new identity, ready to move forward in their new professional role as a PA.

Van Gennep’s (1960) classic treatise on rites of passage mirrors the flow of transition described by Bridges. He viewed "the life of an individual in any society (as being) a series of passages from one age to another and from one occupation to another. Wherever there are fine distinctions among age or occupational groups, progression from one group to the next is accompanied by special acts” (pp. 2-3). When moving from an old status, individuals leave behind what is known and venture into a role that is 
unknown. Van Gennep described this middle stage as "liminal," where liminal is defined as a transitional state or "threshold" (p. 11). Liminality has been described as an individual's transition between the old and new social or cultural status, or between what was and what is becoming. As the new role or status is assumed, the individual is reincorporated into a new social or occupational group (see Figure 2).

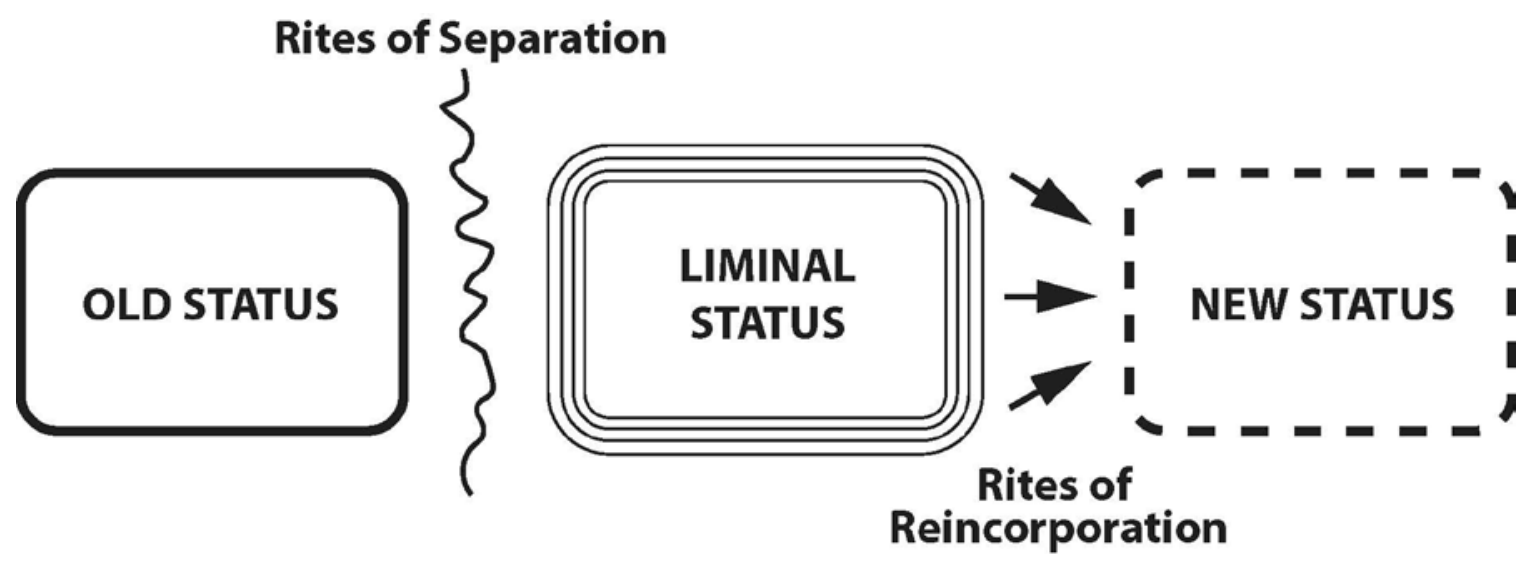

Figure 2. Rites of passage. Adapted from Van Gennep (1960).

Another perspective of transition is described by Schlossberg, Waters, and Goodman (1995) in this way, “A transition, broadly, is any event, or non-event, that results in changed relationships, routines, assumptions and roles . . . A transition may well be a life event that involves gains and losses” (pp. 27-28). In contrast to Bridges, Schlossberg's (1981) Transition Theory does not require a crisis in order to identify a transition-in fact, she advocated that “non-events” (p. 5), such as an expected job promotion that fails to occur, are also capable of altering the perspective of the individual resulting in a transition. Her model of the transition process incorporates phases beginning with moving in, where a newcomer is first confronted with new roles and begins the socialization process. Next is the process of moving through, a period 
described as "betwixt or between" (Schlossberg et al., 1995, p. 52), similar to the Neutral Zone. During this phase, the individual struggles to create new relationships and routines - a time of liminality where the individual undergoing the transition experience suffers from emptiness and confusion. The final phase is moving out, where the individual disengages and separates having completed the transition (see Figure 3).

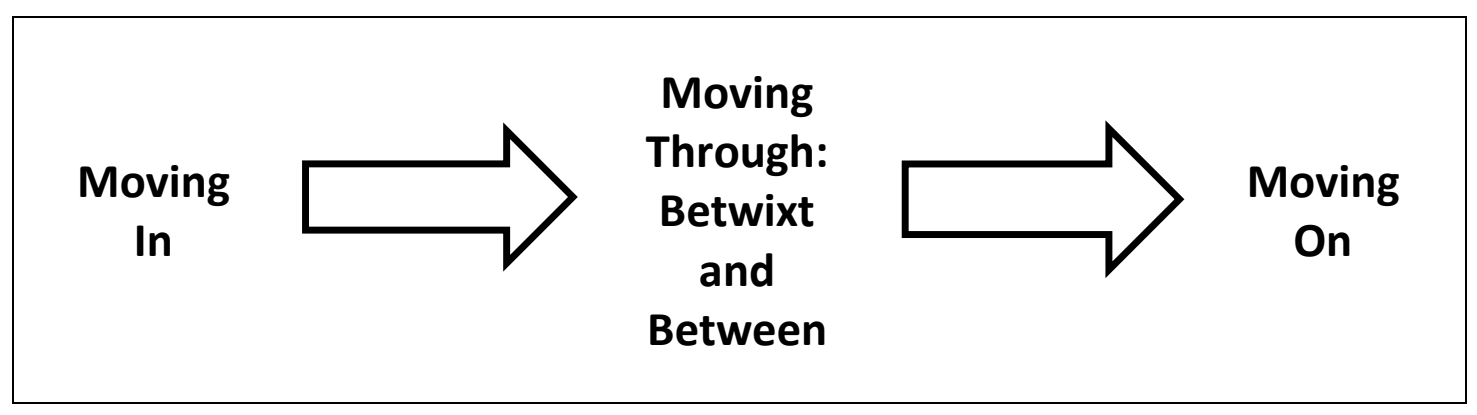

Figure 3. Schlossberg transition theory. Adapted from Schlossberg et al. (1995).

Sargent and Schlossberg (1988) have the belief that adult behavior is controlled by transitions instead of age; that chronological age is not indicative of human development and in fact is a poor predictor of behavior. Our faculty members observe students from a wide range of ages who all seem to be simultaneously experiencing similar transitions. Sargent and Schlossberg's theory could provide some explanation in that it emphasizes the transition as the primary mediator for behavior rather than the age of the individual student. Given that the PA students engage the medical education experience as a cohort and travel through the process together in a lock-step fashion, it makes sense that they would share similar transitional experiences as they are exposed to curricular elements simultaneously. 
The business world also recognizes the role of change in the work environment. Elrod and Tippett (2002) described workplace change as "pervasive-it is a constant and common element that impact humankind individually and organizationally, day in and day out” (p. 273). Much of Bridges’ (1985, 2003) work arose from organizational and individual change he observed in the workplace. The construct of change management centers on the transition of workers and organizations from their current roles and activities into future ones. In order to facilitate that process, there now exists a field of change management defined as "the application of processes and tools to manage the people side of change from a current state to a new future state so the desired results of the change (and expected return on investment) are achieved.” (Hiatt, n.d., para. 19). Elrod and Tippett highlighted the importance of understanding change models in order to illuminate the process of managing change and enhancing the ability of those in leadership roles to cope with the pervasive nature of change. Menninger's morale curve was utilized by Schneider and Goldwasser (1998) in the development of what is described as the classic change curve seen in organizational performance. This particular model is unique in its introduction of the notion that change can be managed in a manner that may flatten out the extreme fluctuations of morale and alleviate some of the distress and despair experienced at the midpoint of the curve while also improving performance. This important concept is relevant to educational programs from an administrative perspective as it implies the ability to assist students through challenging educational endeavors through anticipatory programmatic planning (see Figure 4). 


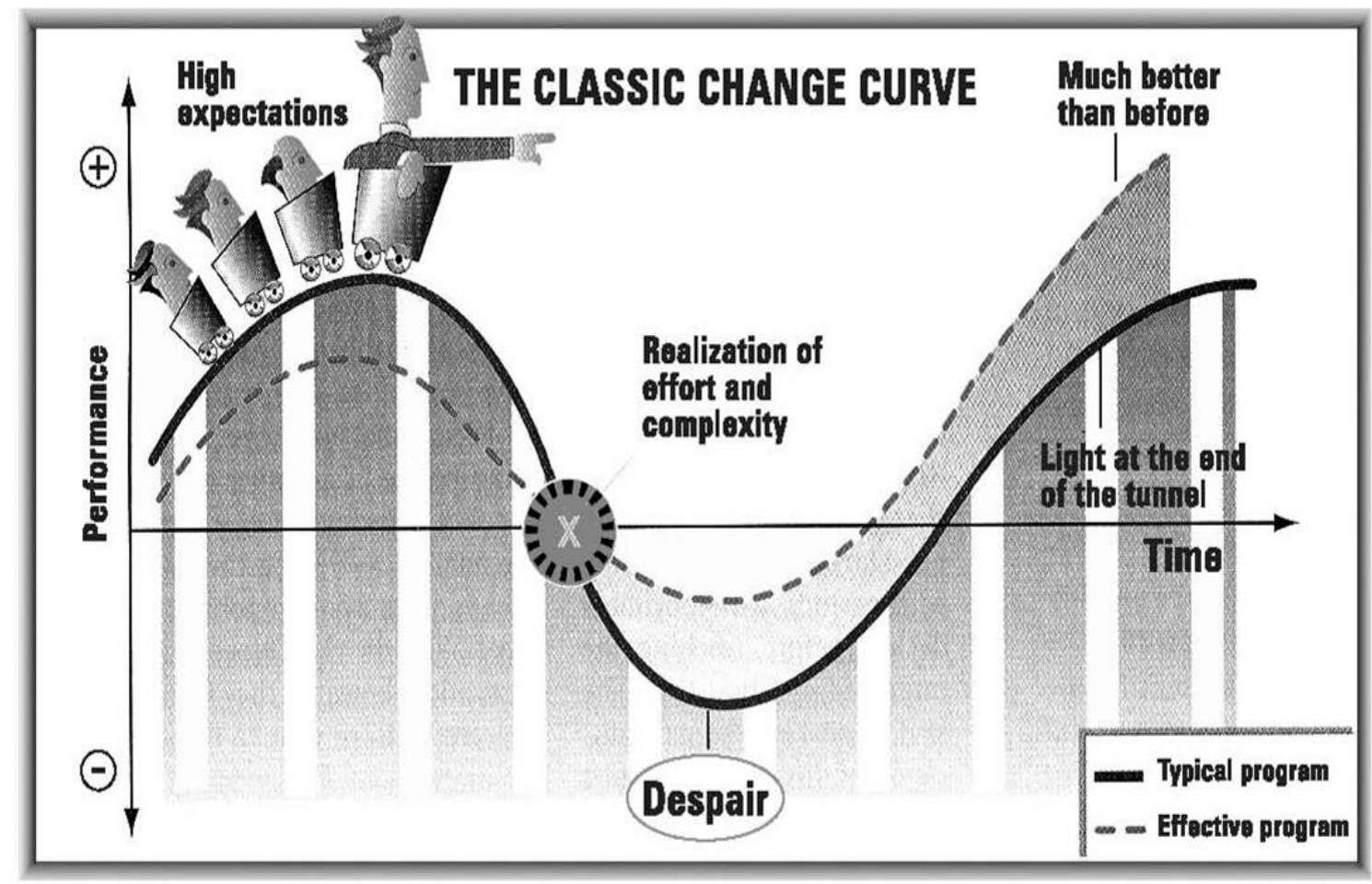

Figure 4. Schneider’s classic change curve. Source: Schneider and Goldwasser (1998, p. 41).

The concept of "The Peter Principle” (Peter \& Hull, 1969) proposes the notion that individuals tend to be promoted from lower levels of business where they have demonstrated competence to higher levels at which they do not. Parker and Lewis (1981) argued, however, that this phenomenon is likely a function of a competent individual being promoted into a new role and subsequently undergoing a challenging transition during which they experience "a degradation of capabilities in the intermediate states of the change process” (Elrod \& Tippett, 2002, p. 273). Orientation and training for a new role often centers around the point of entry, whether a new job or a new educational program, and tends not to extend into the transition itself—a consideration when welcoming newcomers who will likely experience transitions as they acclimate to their 
new environments with new expectations. Conducting orientation activities only at the point of matriculation into a new job or educational endeavor fails to provide continuing support at the time when new hires or new students are most likely to need assistance.

Another example of a transition theory potentially applicable to the PA student experience is found in the cross-cultural literature. Entering into a new culture, whether by traveling to new and foreign locales or assuming a new role in a well-defined profession, involves adaptation to new ways of being.

Culture means the whole complex of traditional behavior which has been developed by the human race and is successively learned by each generation. A culture is less precise. It can mean the forms of traditional behavior which are characteristics of a given society, or of a group of societies, or of a certain race, or of a certain area, or of a certain period of time. (Mead, 1937, p. 17)

Lysgaard (1955) conducted interviews with 200 students from Norway who spent time in the United States on Fulbright travel grants. Through these interviews, he depicted the student adjustment to a foreign society or culture as a U-shaped curve that occurs as a process over time. Although this retrospective study did not seek to identify particular stages within the adjustment, the respondents characterized their experience as one that began with “good initial adjustment, followed by an adjustment 'crisis,' after which good adjustment (was) again achieved” (Lysgaard, 1955, p. 49). Gullahorn and Gullahorn (1963) described a similar pattern of adjustment of foreign students into host cultures as beginning with positive emotions including elation and optimism, followed by a phase of confusion and depression coined culture shock. As students continued their adjustment to the new culture, a developing sense of satisfaction and personal growth appeared-a pattern remarkably similar to that observed by PA program faculty of the first-year PA 
student experience and one that is equally evocative of Menninger's morale curve (see

Figure 5).

FIGURE 1

The U-Curve of Cross-Cultural Adjustment

\section{Degree of Adjustment}

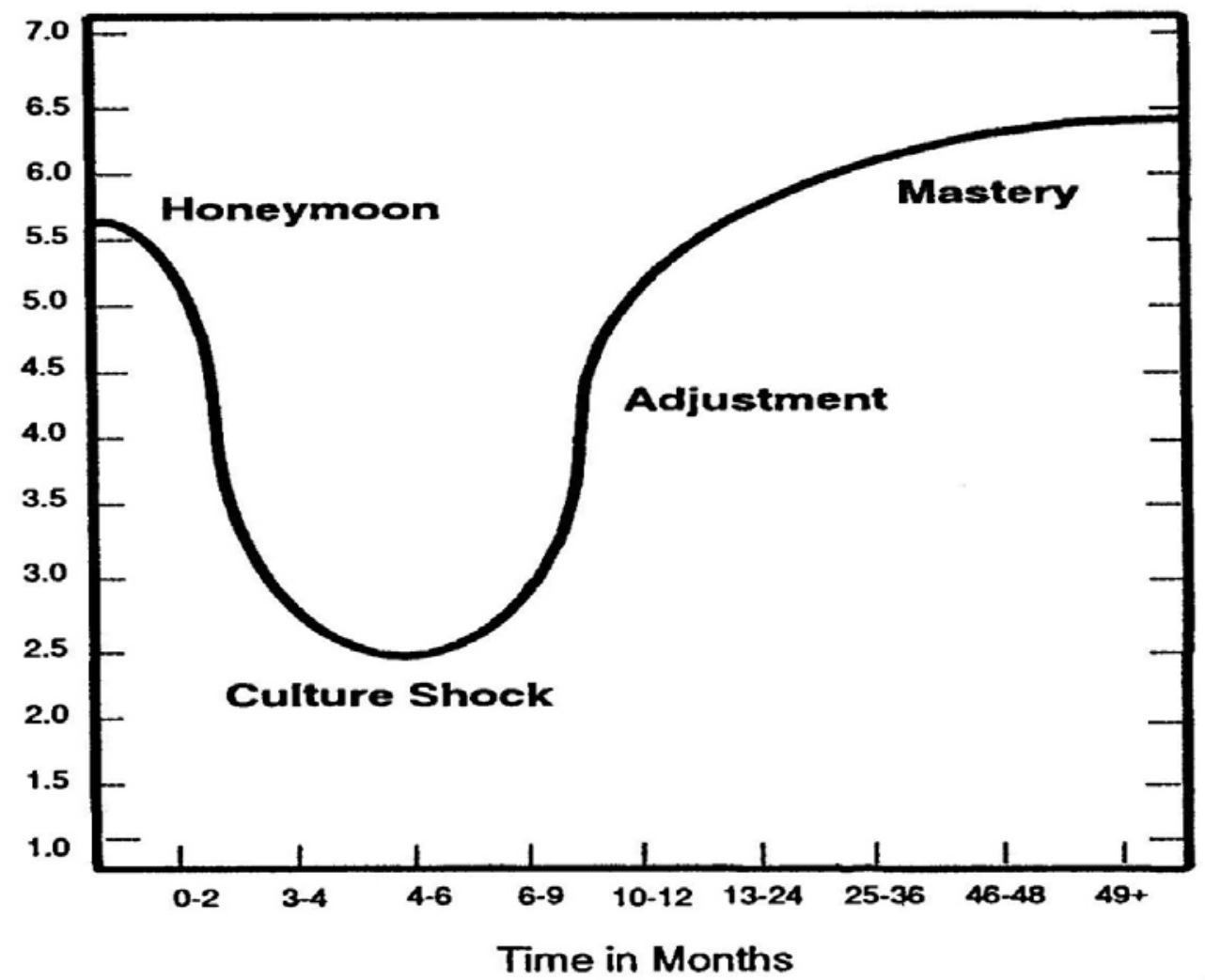

Figure 5. The U-curve of cross-cultural adaption. Source: Black and Mendenhall (1991, p. 227).

In a later review of the literature on the U-curve adjustment hypothesis, Black and Mendenhall (1991) identified a number of cross-cultural studies done utilizing retrospective recall that each concluded with support for the existence of a U-curve pattern of adjustment. One of the criticisms of the U-shaped curve theory identified through this literature review, however, is that it is less a theoretical framework for why 
adjustment occurs in a particular pattern as it is a description of the phases of adjustment. They postulated Bandura's (1977) social learning theory (SLT) as a theoretical foundation for the presence of the U-curve explaining that SLT incorporates both cognitive as well as behavioral aspects of learning: cognitive through mental processing, and behavioral through the execution of activities and behaviors. Engagement in a new culture clearly is a learning experience that requires both learning modalities in order to achieve successful integration.

Adler (1975) wrote that traveling outside of one’s country of origin was not required to experience culture shock and he related the phenomenon to any transitional experience including entering college, being released from prison, getting married or divorced, or other significant changes in role or occupation. The transitional experience could be termed a sojourn, defined by L. Brown and Brown (2009) as “a temporary between-society contact . . . . exposure to a new culture (that) has transformative potential” (pp. 341-342). Utilizing Mead’s (1937) definition of culture, it is possible to label the medical profession as a culture-a closed culture requiring mastery of extensive educational experiences, a specific shared language and expected patterns of behaviors and competencies before newcomers are welcomed into the fold. If the U-shaped curve represents the sojourn, culture shock would be represented by the manifestation of confusion and frustration experienced during the liminal phase of role transition. "Implicit in the conflict and tension posed by the transitional experience lies the potential for authentic growth and development” (Adler, 1975, p. 14). In this sense, the U-curve experience may be descriptive of the emotional experience of the transformational social learning process as students achieve higher levels of personality development. 
There are two additional and seemingly disparate models that identify stages of change similar to those previously discussed — those of teacher attitudes and retirement. Moir (1999) observed more than 1,500 new teachers and identified what she described as stages of a novice teacher's first year; anticipation, survival, disillusionment, rejuvenation and reflection. As seen in the diagram below, the graphic representation of this year-long process is similar in construction to Menninger's (1988) morale curve, Schneider’s (Schneider \& Goldwasser, 1998) classic change curve, and Gullahorn’s (Gullahorn \& Gullahorn, 1963) U-curve of cross cultural adaptation (see Figure 6).

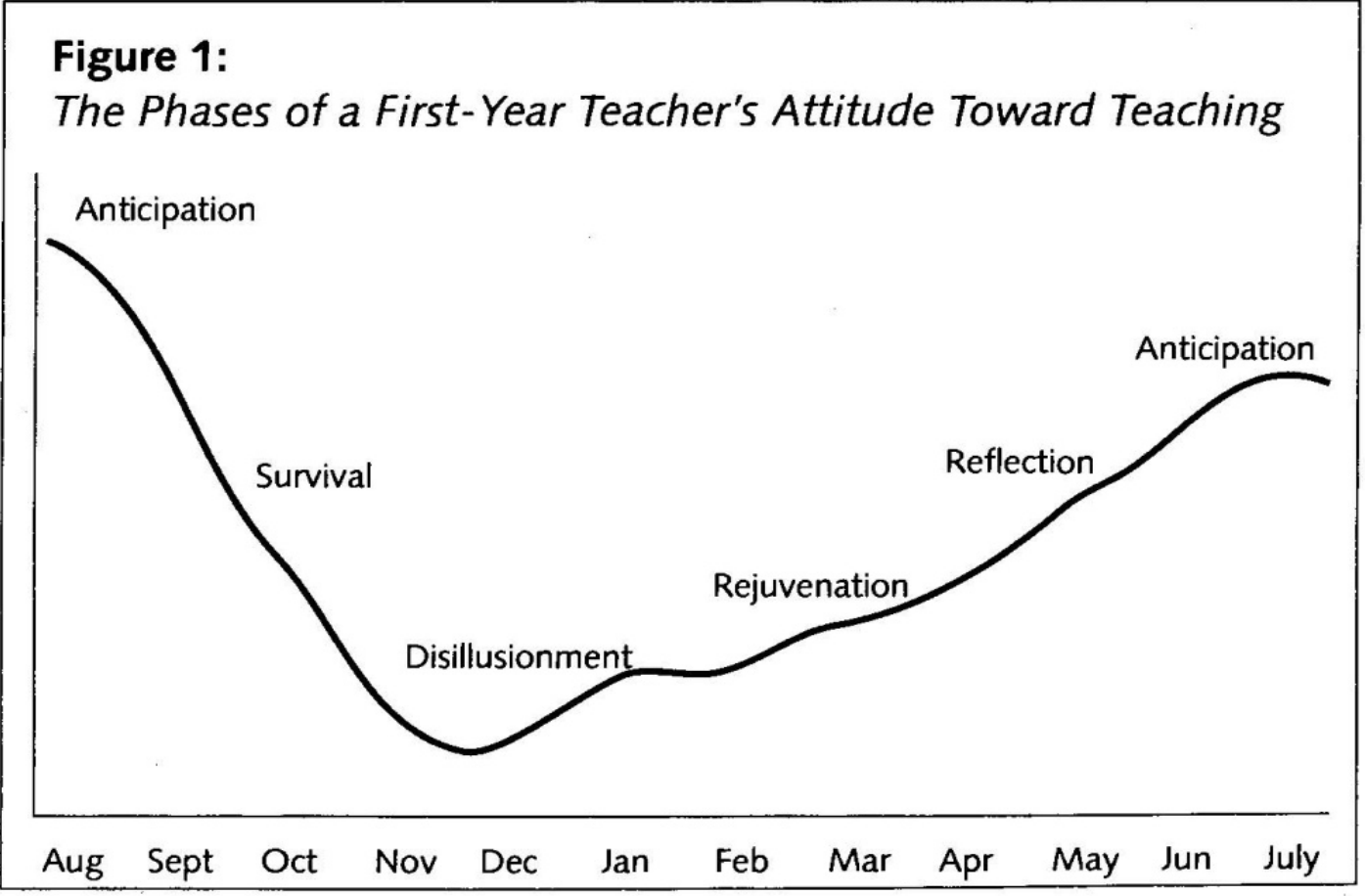

Figure 6. Phases of a first-year teacher's attitude toward teaching. Source: Moir (1999).

A final example comes from the work of Atchley, who in 1976 described the sociology of retirement. He depicted retirement as a role assumed by an individual who is leaving a defined work identity and beginning a new phase in life. Prior to his 
investigation, little attention had been paid to the dynamics of role relationships in older individuals, but as the population as a whole has enjoyed an increasingly long life span over the last several decades, attention to the activities of this social role is warranted. Atchley identified role phases beginning with a prelude to retirement he labeled as preretirement. Once the individual actually stopped their formal work role and actively assumed the retirement role, they underwent a honeymoon phase similar in description to Menninger's Crisis of Arrival. This was followed by the disenchantment phase where the struggles of adjustment became most pronounced and the distinctions between expectations and reality were clarified. Retirees were then said to pull themselves together during the reorientation phase which then transitioned into the stability phase as they adapt to their new role. In Atchley's view, the termination phase associated with the change process of retirement is represented by death. Although he did not illustrate his proposed phase process through the utilization of a line drawing representing the upsand-downs of the longitudinal experience, the descriptive elements of each of these phases are remarkable similar to previously described models (see Figure 7). Because retirement is such an individual construct given that there is no universal period at which everyone must begin the process, Atchley believed the phases of retirement could not be tied to chronological time frames or ages and instead likened them to a "typical progression of processes” (p. 71). 


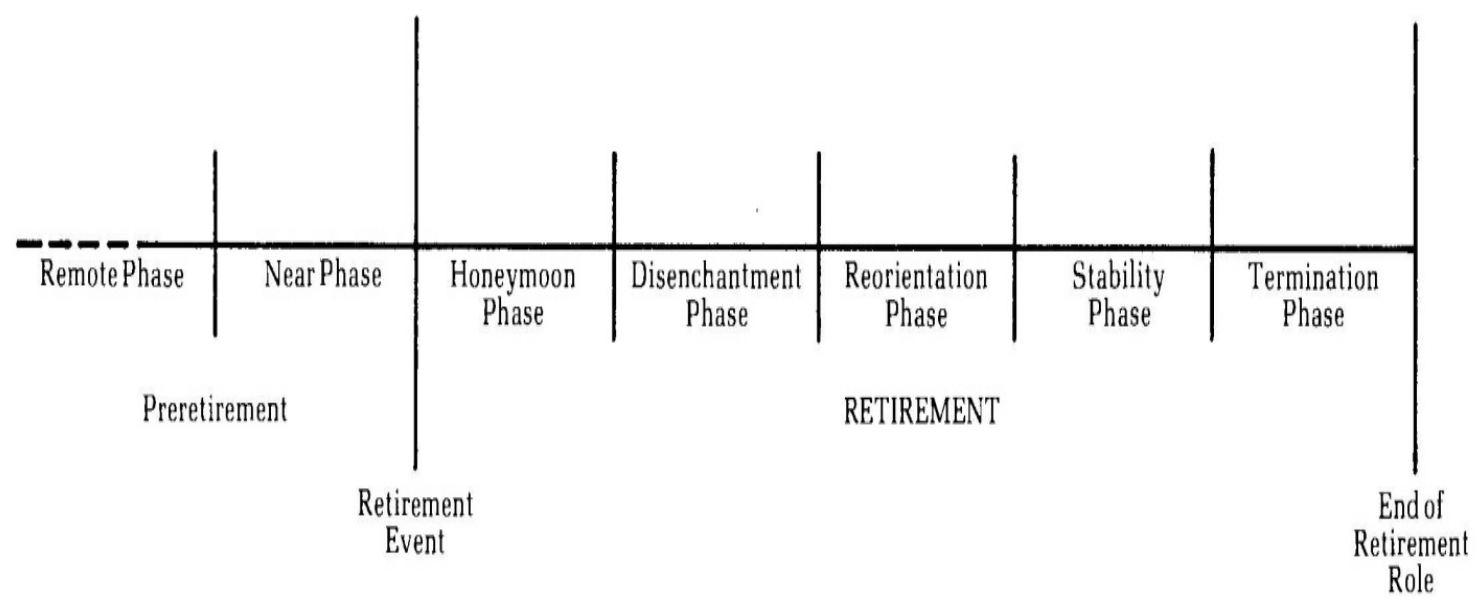

Figure 2. Phases of Retirement

Figure 7. Atchley’s phases of retirement. Source: Atchley (1976).

There appears to be a great deal of consistency regarding the description of change and transformation across several diverse disciplines of study, with most conforming to a general three-stage approach to the way in which individuals respond when confronted with transition. These models are each descriptive of the observed experience of the PA cohorts as they move through their didactic medical education, yet this model has not been consistently applied to formal educational processes in general nor to medical education programs in particular. Education and learning contribute to the transformation of individuals, and regardless of the type of transitional event, learning constitutes a prominent role in the process of personal and professional development. To further explore the concept of transformation as a function of learning, the next section discusses Mezirow’s (2000) Transformational Learning Theory to explore another perspective of the learning process as experienced by PA students. 


\section{Transformational Learning}

Acknowledgement of the transformational nature of the professional development process ingrained in medical education programs would suggest that the application of these theories to the academic environment would not only be appropriate but instructive as well. The seminal theoretical model describing transformative change in education is credited to Mezirow (2000), who has depicted transformation as a process where meaning is explored and clarified, culminating in a reintegration into a new life with a new understanding of reality—the perfect description of socialization into a new professional role. Although not labeled as a change process per se, his constructivedevelopmental theory of transformational learning nonetheless succinctly describes the PA student experience at an academic health center in the Pacific Northwest, beginning with matriculation into the program and continuing through what he described as the 10 steps during which transformation occurs (see Table 1).

Mezirow’s (2000) model of transformative learning describes an incremental process in which a series of small changes in points of view ultimately result in a "transformation in habit of mind" (p. 21). We observe these changes taking place continually throughout the 2-year educational process. For example, the acquisition of physical examination skills which seems so challenging to students at the beginning of the program becomes second nature after repeated practice and numerous patient encounters. Students are often unaware of this incremental growth and express surprise at how easy content seems later in their education when compared to how difficult they found it as a new student. As they are learning these skills, they are not only constructing knowledge but transforming themselves into clinicians. Beyond just developing skills, 
Table 1

Mezirow's Transformative Learning Theory Applied to PA Education

\begin{tabular}{|c|c|}
\hline Mezirow’s (2000) 10 Steps of Transformation & PA Student Activities and Timeline \\
\hline 1. Experiencing a disorienting dilemma & $\begin{array}{l}\text { Matriculation into the PA program initiates the } \\
\text { exciting challenge of adjustment into a new } \\
\text { academic environment }\end{array}$ \\
\hline $\begin{array}{l}\text { 2. Self-examination with feelings of fear, anger, } \\
\text { guilt or shame }\end{array}$ & $\begin{array}{l}\text { Excitement and exhilaration is short-lived as old } \\
\text { roles are abandoned and a dawning recognition of } \\
\text { incompetence and loss of former identity develops }\end{array}$ \\
\hline 3. Critical assessment of assumptions & $\begin{array}{l}\text { Despite a prior record of academic success, students } \\
\text { now have a fear of failure and ambivalence about the } \\
\text { decision to start such a process-questions arise } \\
\text { about their ability to succeed, and whether this was } \\
\text { the right choice to have made }\end{array}$ \\
\hline $\begin{array}{l}\text { 4. Recognition that one's discontent and the } \\
\text { process of transformation are shared }\end{array}$ & $\begin{array}{l}\text { Development of the cohort and its shared morale- } \\
\text { "We are all in this together!" }\end{array}$ \\
\hline $\begin{array}{l}\text { 5. Exploration of options for new roles, } \\
\text { relationships and actions }\end{array}$ & $\begin{array}{l}\text { Taking on the role of a PA through interactive and } \\
\text { collaborative learning activities }\end{array}$ \\
\hline 6. Planning a course of action & $\begin{array}{l}\text { Following the proscribed educational curriculum, } \\
\text { students work toward achieving each of the required } \\
\text { programmatic milestones }\end{array}$ \\
\hline $\begin{array}{l}\text { 7. Acquiring knowledge and skills for } \\
\text { implementing one's plans }\end{array}$ & $\begin{array}{l}\text { Transitioning from the novice stage to advanced } \\
\text { beginner-the early development of beginning } \\
\text { expertise as a didactic year student occurs with the } \\
\text { completion of each educational block }\end{array}$ \\
\hline 8. Provisional trying of new roles & $\begin{array}{l}\text { Participation in supervised clinical apprenticeship } \\
\text { activities and activities with standardized patients } \\
\text { provides opportunities to practice }\end{array}$ \\
\hline $\begin{array}{l}\text { 9. Building competence and self-confidence in } \\
\text { new roles and relationships }\end{array}$ & $\begin{array}{l}\text { Begins developing late in the first year as } \\
\text { competence increases with repeated practice and } \\
\text { enhanced content knowledge }\end{array}$ \\
\hline $\begin{array}{l}\text { 10. Reintegrating into one's life on the basis of } \\
\text { conditions dictated by one's new perspective }\end{array}$ & $\begin{array}{l}\text { Departure from the didactic portion of the program } \\
\text { as they enter the clinical phase-students tell us they } \\
\text { are "changed” having assimilated into the entry level } \\
\text { of the culture of medicine }\end{array}$ \\
\hline
\end{tabular}


students also expand their metacognitive abilities as they move through a developmental continuum. From the novice phase where rule-oriented behavior presides, to the increasing levels of proficiency inherent in the ascent toward the competency of the expert role (Daley, 1999; Dreyfus, 2004), students learn relevancy and “meaningful situational components” (Benner, 1982, p. 403) with which to address medical problems. In addition to incremental transformations which may go unnoticed by students, Mezirow (2000) also described epochal transformations: those which are sudden, dramatic and reorienting in nature. Patient care involves challenging circumstances including sickness, suffering and death; these emotional encounters provide an avenue for students to reexamine their ways of knowing and they are often transformed by these experiences.

Mezirow (2000), Bridges (1985, 2003), Van Gennep (1960) and Lewin (1947) each proposed models with a similar course of events, beginning with a dramatic reorientation of a student’s perspective, ala Mezirow’s “disorienting dilemma,” Bridges'(2003) ending, losing, letting go, Van Gennep’s rites of separation and Lewin’s unfreezing. While Mezirow's theory explains the effects of transformational learning on students through his description of the process of acquiring skills and assuming new roles, Bridges (2003) and Lewin developed their theories of change from a perspective of group dynamics and organizational development. Van Gennep’s theory arose through participant observation of socialization rites and rituals among a variety of cultures. All, however, highlight a similar process during which individuals replace their old ways of thinking with new points of view: a process with a predictable beginning, middle and end. Each of these models also highlights the midpoint of the transformation process as 
the most emotionally volatile. If Menninger’s (1975) Morale Curve represents a graphic depiction of transformational learning involving personal change and growth, the emotional lability of the change process on participants might be predictable based on the life cycle of the educational process. Yet the effects of programs alone cannot explain all the manifestations of psychological distress among students. The next section elaborates additional issues impacting the emotional nature of transformative programs in order to further describe the underpinnings of the student experience.

\section{Mediators of Psychological Distress in Medical Education}

Just as seen with members of the military or Peace Corps volunteers, there are a number of moderating factors that can contribute to fluctuations in individual student morale over time (Estes \& Wilensky, 1978; Parkerson, Broadhead, \& Tse, 1990). In addition to the influential effects of the educational program itself, life events and personal circumstances can impact the ability of students to fully participate in their medical education. These life events might include financial pressures, a death in the family or an unexpected pregnancy; each of these represents a significant change in stability. Myers, Lindenthal, and Pepper (1975) conducted a longitudinal interview study of adults and identified a relationship between life events and psychiatric symptoms. They found that the greater the number of life events, defined as role transformation, changes in status or employment, or imposition of pain, the greater the possibility of psychiatric difficulties. Although the Myers et al. study did not investigate subjects engaged in a medical education program specifically, the process of medical education involves socialization into a new role and as such qualifies as a life event according to the parameters defined by these authors. Additional life events experienced by PA students 
can include moving to a new location to attend school, loss of income in conjunction with accumulation of significant educational debt, changes in marital or relationship status, and loss of support systems to name just a few. The combined effects of role socialization, involvement in an intensive educational process and personal encumbrances can challenge students to maintain resilience and identify coping mechanisms with which to successfully navigate this taxing time. As a result, they may be at greater risk for psychological distress while undergoing this transformational process.

Literature describing transformative growth and development in adult education is abundant (Burton, 2006; Choy, 2009; Cranton, 1998; Robertson, 1996, 1997; Sandars, 2006; Tennant, 1993), but scant attention has been directed toward the concept of cohort student morale in professional medical education, particularly during the didactic phase where the cohort experience is most prominent (Woloschuk et al., 2004). Investigations into student mental health have been undertaken among some health professions students, and identification of psychological challenges during the medical education process is ongoing. So what are some of the psychological challenges experienced by students during this intensive educational process? The next section provides a brief overview of what is known about the ways in which medical education affects students’ psychological well-being.

\section{Psychological Distress During the Process of Medical Education}

Although there is limited formal research highlighting PA education specifically, the literature abounds with information about student psychological distress while engaged in other types of professional education including medicine, dentistry, nursing 
and law (Cardall et al., 2008; D. C. Clark \& Zeldow, 1988; Dickstein, Stephenson, \& Hinz, 1990; Dyrbye, Thomas, \& Shanafelt, 2006; Helmers, Danoff, Steinert, Leyton, \& Young, 1997; Roberts, 2010). It is well known that many students experience stress as part of a medical education process (Finkelstein et al., 2007; Henning et al., 1998; Schmitter et al., 2008), and stress has been recognized to have a negative effect on academic performance (Spiegel et al., 1986), health and psychological well-being (Helmers et al., 1997). High rates of stress, anxiety and depression have been reported in numerous articles on medical student mental health (D. C. Clark \& Zeldow, 1988; Dahlin, Joneborg, \& Runeson, 2005; Dyrbye, Thomas, Huntington et al., 2006; Schwenk, Davis, \& Wimsatt, 2010; Vitaliano et al., 1984). Most students respond appropriately and manage the stress effectively; some, however, descend into demoralization and depression and may be at risk for suicidality (Rickelman, 2002; Schwenk et al., 2010). Dyrbye, Thomas, Huntington et al. (2006) confirmed levels of psychological distress among medical students higher than that of the general population, and Spiegel et al. (1986) noted an inverse relationship between morale and interpersonal stress for male and female students, suggesting that as interpersonal stress increased, morale declined.

\section{The Relationship Between Psychological Distress, Empathy and Professional Behavior}

Psychological distress has far reaching effects, including effects on empathy and professional behavior (Brazeau, Schroeder, Rovi, \& Boyd, 2010; Hojat et al., 2004;

Krasner et al., 2009; Michalec, 2010). Dyrbye et al. (2010) identified medical student distress as a significant factor in outcomes related to professional behaviors. Wear et al. (2006) reported increasing levels of cynicism as students advanced through medical 
school, and cynicism has been associated with "ethical erosion” (p. 454). Burnout, described by Maslach (1982) as a syndrome of emotional exhaustion, depersonalization of others and feelings of reduced personal accomplishments, has been linked to a decline in empathy. The state of burnout is suggestive of the description of subjective incompetence (de Figueiredo, 1983) and reduced self-efficacy (Bandura, 1982), particularly as they coincide with overwhelming quantities of work. The impact of burnout is significant as medical students who identify themselves as having burnout are less likely to report themselves as altruistic (Dyrbye et al., 2010)—a key component of patient care. In one study among seven medical schools (Dyrbye et al., 2010), burnout was the only aspect of personal distress that was found to be independently correlated to unprofessional behaviors and reduced altruism. Loss of altruism and depersonalization of others can result in loss of empathy, which has been shown to result in a decreased willingness of students to care for the chronically ill (Woloschuk et al., 2004). Students do not matriculate into medical education programs already in a cynical state, yet cynicism develops into an increasingly significant problem over the course of the medical education process. A single study investigating empathy among PA students confirms a decline over time during the PA education process (Mandel et al.) and highlights the need to further explore cynicism and burnout among PA students, psychological constructs that could be closely related to the degree of distress experienced during a transformational education process.

Although it is clear from the literature that the process of medical education can create psychological distress with its attendant effects on empathy, it is challenging to tease out the etiology of this distress due to the many contributing factors. Certainly the 
educational workload itself contributes to the degree of stress experienced by students, but other dynamics may be involved as well. Regardless of the cause, there is a convincing body of information confirming the presence of psychological distress among health professions students. Given the similarity of the educational model and the abbreviated timeframe in which PA students are expected to internalize a massive amount of complex medical information, it is only reasonable to assume that PA students are likely to have equal, if not greater, amounts of psychological distress as their medical student colleagues.

The process of medical education is rigorous and stressful yet should not be inhumane, nor should it erode student empathy, altruism or resilience. To improve the medical education experience by reducing stress, demoralization and low morale while maintaining academic standards, programs must be aware of the impact the educational process has on students and make changes accordingly. In order to do so effectively however, the scope of the problem must first be clearly delineated. Given the limited data describing the well-being of PA students, investigation into their psychological status and the effect that education might have on that status is warranted. The next section describes a qualitative naturalistic research approach eliciting the voice of the students in order to elaborate their experience of the medical education process. 


\section{CHAPTER 3}

\section{METHODOLOGY}

\section{Aim of the Study}

The medical education process to become a PA is rigorous, time-consuming and transformative, yet to date little is known about its effects on PA students from the perspective of the student. There is a dearth of information on an important experience that affects thousands of students who undertake this educational role transformation process to become PAs each year. The aim of this study was to explore the experience of PA education at an academic health center on the West Coast, and identify common themes ascribed to those experiences from the student perspective. An additional goal was to enhance understanding about this understudied group while simultaneously giving students a voice in the educational process in which they are engaged. The overarching research questions were:

- What is the student experience of the didactic phase of PA education?

- In what ways do these experiences affect students?

- How do those experiences affect student mood and morale?

This chapter describes the methodological considerations and decisions that were made in designing and carrying out this study.

\section{Research Approach}

In choosing a research paradigm on which to theoretically frame this inquiry, it was important to keep in mind the goal of the investigation which was to elucidate the 
student's experience and the process by which they undergo personal and professional transformation into that of a medical professional. The process of role development and role identity has been described as a socially constructed reality (Roeser et al., 2006), and as the educational process to become a medical professional involves both, this investigation cannot be adequately characterized by a positivist approach (Guba \& Lincoln, 2005). Instead, constructivism provides a platform for the human voice description of the experience of this transformative process. The paradigm of social constructivism provides a worldview from which one can begin to understand the meaning of a common experience, and the process of medical education-particularly in a small cohesive cohort simultaneously engaged in a lock step curricula—creates a structure in which students come together and socialize into a new professional role through common experiences. The perspective of this experience is subjective and based on the multiple realities of the student participants. Utilizing this ontological perspective, a qualitative research design was the most appropriate means by which to identify the different perspectives of individuals as well to elucidate the perceptions of their lived realities.

Within the construct of qualitative research design, there are a variety of methodological options from which to choose. Naturalistic methods seek to describe “naturally occurring activity in natural settings” (Hatch, 2002, p. 26), an approach which concisely describes the goal of this project. Lincoln and Guba (1985) identified naturalistic research methods as most appropriate for inquiry into constructivist paradigms, and Hatch (2002) stated that they can be used to "reconstruct the constructions participants use to make sense of their worlds” (p. 15). Further, a 
naturalistic approach is uniquely suited to data collection through the use of observation and interviewing techniques in order to explore and understand the fundamental nature of the student experience of medical education process. While PA program faculty engage in casual participant observation on a regular basis, and while it is possible to have an “observational understanding” (Seidman, 1998, p. 3) of an individual one is observing, the missing component is that of the students and their individual perspectives on the experience. For that reason, I chose interviewing as the ideal method by which to elicit the voice of the student.

Qualitative interviewing provides an opportunity to listen to individuals describe their worlds. Those with communal backgrounds have shared understandings that constitute culture, and interviewing can explore shared culture as well as specific events (Rubin \& Rubin, 1995). Certainly individual and collective events contribute to the educational experience as a whole. While I suspect that PA cohorts do form a culture within each class, my primary interest is more topical in nature; in other words, how might students describe their individual experiences as members of a cohort undertaking the didactic phase of PA education.

When determining the most appropriate type of interview to utilize it was important to consider both individual and group interview techniques. While the unit of analysis in this study is the individual PA student, students do share common educational experiences as members of a class cohort, and as such they can each contribute their individual perspectives of the lived group phenomenon to the conversation. For this reason, focus group interviews provided the greatest opportunity to explore the student individual experience of a group process. A focus group interview according to Kreuger 
(1988) is a "carefully planned discussion designed to obtain perceptions on a defined area of interest in a permissive and nonthreatening environment” (p. 18). Focus group interviews provide an opportunity for abundant discussion through group interaction, and provide data on individual realities within the context of a group. Discussion among members of the group can offer elaboration on the interpretation of events. This type of data collection allows students to explore their experiences retrospectively from a metacognitive perspective in the company of others who have had common educational experiences. The homogeneity of the group is a key construct underpinning the purpose of the study (Kingry, Tiedje, \& Friedman, 1990). While a focus group interview is to some degree an unnatural social encounter, in this case it represented a dynamic similar to that which takes place within the PA student cohort itself during regular school activities, and did so in a setting natural to these students. Because participating students knew each other well, these focus groups had an element of intimacy more common to family interviews (Olson, 2011), which may have enhanced participants’ willingness to speak when compared to focus groups utilizing strangers as participants. In addition, focus groups provide an advantage over individual interviews in that they allow the researcher to observe interactions between participants during the interview process on the topics of interest to the researcher (Morgan \& Spanish, 1984). In fact, Casey and Drueger (1988) highlighted the use of the group setting as an opportunity to collect data that might otherwise be unavailable without the group interaction.

Frey and Fontana (1991) described group interviews as particularly helpful in the process of "indefinite triangulation” (p. 177) in which ideas are bounced back-and-forth between individuals and modified by the group, leading to a greater elaboration of 
statements beyond that provided by a single individual. The "polyphonic” (p. 178) data collected represents a broader spectrum of participant opinions while diffusing the influence of the interviewer within the group setting.

Because the aim of the study was to elicit the student experience of the process of didactic education, focus group questioning required a broad and open-ended approach designed to draw out participant opinions and shared stories. The focus group sessions were digitally recorded, professionally transcribed, and systematically analyzed using a process of open coding content analysis described more fully later.

\section{Study Participants}

As the primary goal of this study was to clarify the student experience of a medical education process, a purposive sample of individuals who had participated in that educational process was required. Study participants were comprised of a volunteer sample of the members of the PA graduating class of 2014. These representatives of the 38 member student cohort had completed the didactic phase at the time of the study and were able to retrospectively reflect on their recent experiences as first-year students-a perspective unavailable to students still engaged in that first-year experience and one that may be too remote for accurate reflection after graduation. All interview subjects fit Rubin and Rubin's (1995) three criteria for appropriate interview candidates: they were knowledgeable about the didactic educational process through their direct participation in the process, as volunteers they demonstrated their willingness to participate, and finally, as our applicant pool is a national one, participants reflected a variety of regional and gender viewpoints within the class cohort itself. Recruitment for student participants took place via email invitation. Addressing Morgan’s (1997) general recommendations for 
focus group size, 10 students ultimately self-identified and were confirmed for voluntary participation in both of the group interviews. Participants agreed to commit to attending both sessions in order to enhance continuity and allow for discussion based on both interactions.

\section{Institutional Review Board Approval and Informed Consent}

Expedited Institutional Review Board (IRB) approval was obtained for this qualitative research project conducted on campus at an academic health center on the West Coast. Informed consent explaining the purpose of the study, its expected duration, foreseeable risks or benefits, and the voluntary nature of participation was obtained from all participants prior to the start of the initial focus group session. A copy of the signed document was provided to participants.

As participants within the focus group were known to the researcher, the scribe and to each other, anonymity was not possible; however, the audio recordings, transcripts and all subsequent results have been kept secured and confidential in a locked file cabinet within a locked university faculty office. Digital recordings of the two focus group sessions were electronically transmitted to a professional transcriptionist via a secure university email system which resulted in anonymized transcripts with comments that could not be attributable to any individual.

\section{Methods}

Study volunteers participated in two face-to-face focus group sessions with other members of their own cohort during two different held several weeks apart. These sessions lasted approximately 90 minutes each and were held in a comfortable conference room familiar to all student participants. Refreshments were provided but participants 
received no other incentives. After obtaining informed consent, the researcher moderated a discussion about the student experience using the following IRB-approved focus group script with broad general questions about the student experience of PA education and the phenomena of mood and morale during the didactic year:

1. Talk about your experience as a student in the didactic year of this program.

a. Did your experience change over time from when you first started the program until the end of the first year? If so, at what points did you notice the change/s?

2. What was it like being part of a cohort of students during that first year?

3. Some people believe that groups develop their own morale after being together for a time. Do you agree or not? If so, how would you describe the morale of your group?

a. Did the group morale change over time? If so, were there certain times when this was noticeable?

b. Were there ever times that the group morale differed from your own? If so, when were those times?

4. Talk about your sense of the overall experience now that the didactic year is behind you.

The discussion was digitally audio-recorded. A scribe was in attendance and took anonymized notes on interactions within the group. At the conclusion of the discussion, participants were asked to complete an anonymous written reflection on their thoughts about the focus group experience and any aspect of their educational experience on which they wished to elaborate. These written responses allowed individuals to expound on thoughts and feelings that arose during the group discussions related to their shared experiences as PA students, including thoughts they may have withheld due to concerns about conformity within the group (Morgan, 1997). Affording participants the chance to 
speak individually as well as part of a group provided the broadest opportunity to fully share their experiences.

The audio recording of the initial focus group session was professionally transcribed by a qualified transcriptionist resulting in formal de-identified transcript that guided the development of the second focus group session. Keywords, expressions and themes identified in the initial meeting drove the development of the script used for the second focus group. Thematic topics for the second focus group meeting included:

1. The emotional nature of the process beginning to end.

2. Loss of confidence and feeling incompetent

3. The experience of feeling “different” from others outside the cohort-having a different language and feeling set apart from others.

4. The importance of the cohort in individual and group success.

5. Accomplishments and a new identity

The second focus group session was conducted in the same fashion as the initial session.

At the conclusion of both focus group sessions, anonymous session transcriptions were reviewed by the researcher and underwent microanalysis to identify general initial categories by which to conceptualize common outcomes and experiences reported by students. Through a process of manual open coding, properties and dimensions of these categories were developed as a means of distinguishing different attributes of the data, which were then reassembled through axial coding around those identified themes in order to describe the phenomenon of the student experience as elaborated by the focus group discussion. The analysis of the content is more fully described in the next section. 


\section{Data Analysis}

Focus group interviews provided an entrée into the essence of the student experience of the cohort process to become a PA. Transforming the narrative qualitative interview data into analyzable information, however, requires condensation and objective coding to make sense of the data. Content analysis is a qualitative analytic technique by which text data collected during a naturalistic research paradigm can be examined objectively with the goal of methodically evaluating characteristics of the transcripts in order to identify meaningful patterns and relationships (Hsieh \& Shannon, 2005). Downe-Wambolt (1992) described the goal of content analysis as providing "knowledge and understanding of the phenomenon under study" (p. 314). Because the very nature of qualitative data is that it can be interpreted to have multiple meanings based on subjective interpretation, it is critical that the findings be as trustworthy as possible in order to have credibility and the potential for transferability (Graneheim \& Lundman, 2004; Morrow, 2005). This is particularly important in this case given the lack of other data about this educational phenomenon, so special care in identifying salient meaning units and themes was paramount.

One of the considerations when using content analysis as an analytic tool is whether to utilize the process to develop a quantitative assessment of the text data, or focus on the qualitative nature of the data. In the former, text data is recorded into categories and subsequently analyzed using statistical measures. Hsieh and Shannon (2005) described this as "quantitative analysis of qualitative data" (p. 1278). In this instance, I was more interested in the meaning of the student experience rather than a numeric accounting of the experience. For this reason, I chose to focus on conventional 
content analysis to describe the phenomenon of the student experience (Hsieh \& Shannon 2005). Another consideration when conducting content analysis is whether to focus on manifest or latent content. Graneheim and Lundman (2004) described manifest content as that which is visible and obvious within the text itself. Latent content, on the other hand, refers to the underlying meaning of the text. Because this inquiry is primarily aimed at eliciting and understanding the student's experience over an entire year, I chose to limit the scope of this study to the manifest aspect of the content-while recognizing the limitations associated with leaving out the latent content. Analysis was conducted on the compilation of the two focus group transcripts; together they are large enough to be considered a stand-alone whole on a defined phenomenon, while at the same time being manageable enough in size as to not be overwhelming.

At the culmination of the focus group meetings, transcripts were reviewed several times to get an overall sense of the "whole.” Student quotations were identified as meaning units, and the meaning units were sorted into content areas and categories with shared commonalities (Krippendorff, 1980; Lindstrom, Hamberg, \& Johansson, 2011). Significant statements and overarching themes were identified in order to systematically analyze the data from which to describe the student experience of the didactic phase of PA education. Emerging themes were then modified and condensed, after which they were reviewed to confirm, reject or reconsider the coding labels. Hand written comments were anonymously transcribed and evaluated for significant statements to provide another perspective from which to understand the effects of the medical education process. 


\section{Methodological Considerations and Potential Research Bias}

In addition to determining the appropriate subjects for this inquiry, it was also important to identify the appropriate person to serve as the focus group moderator. In my role as the Academic Coordinator of the didactic cohort of PA students, I engage in participant observation on a daily basis. Immersion in the day-to-day lives of my students has provided many opportunities for informal realist ethnographic inquiry, and these daily interactions have served to fuel my continued interest in understanding the student experience of the educational program I am responsible for delivering. My role in the didactic phase uniquely positions me to understand the context of the student experience from a holistic perspective, and for that reason I believe I was best suited to moderate the focus group activities.

As the Academic Coordinator of the program, I am responsible for the didactic education of all PA students during their first year. In that role I am a course director for courses, I teach, and I maintain a significant role in assessing and grading students. This position represents one of power over students and raises concern about potential conflict of interest. It is for this reason that I chose to utilize student participants who had completed the didactic year, for once they have advanced to the clinical phase of the program I no longer control their movement through the process, nor do I have a significant role in grading or assessment. The differential that exists between students and faculty remains of course, but the power disparity of the academic year is no longer a substantial source of conflict. Additionally, because students volunteered to participate in this research study, anyone who felt uncomfortable about that participation had the ability to not to be involved, therefore eliminating any potential concern. 
In addition to acknowledging issues associated with power, from an axiological perspective I also acknowledge the bias I hold regarding the patterns of mood and morale based on observations among student cohort, but it is a bias shared by others (e.g., Seashore, 1975). Having once been a PA student and now functioning in the role of a medical educator educational administrator responsible for overseeing the didactic curriculum for PA students, I am fully integrated into the experience. Being imbedded into this educational culture, however, does not negate the important need to enhance our understanding of the consequences of this process from a variety of different perspectives. Concerns about the influence of the moderator on the group interaction are valid, but these concerns are "hardly unique to focus groups because the researcher influences all but the most unobtrusive social science methods” (Morgan, 1997, p. 14) .

To limit subjectivity based on my interactions with the study participants and familiarity with the educational process itself, I sought to engage in participant checks, asking for clarification and elaboration into textural meaning. Because memory changes with time, I did not send out transcripts wholesale, but instead sought feedback in those areas needing additional clarity. Additionally, I shared focus group comments with fellow faculty members to seek out opinions regarding their impressions of student perspectives regarding mood and morale over the course of the didactic year to assure that I was realistically reflecting the student experience. While acting as researcher/moderator, I strove to maintain a vigilant awareness of my role in this process to minimize the potential for bias. 


\section{Summary}

Through extended observations of class cohorts over a number of years, changes in student mood and morale seem to take place at critical junctures in the educational process, yet no empirical evidence exists to support these observations, nor have student impressions of the educational process previously been elaborated. Utilizing a naturalistic research approach incorporating focus group interviews at a West Coast PA program, student perceptions about their educational experience were gathered and subjected to qualitative content analysis. In the next chapter, the analysis of the focus group data is presented and discussed. 


\section{CHAPTER 4}

\section{RESULTS AND DISCUSSION}

The purpose of this study was to elucidate the student experience during the didactic phase of PA education, a medical education immersion process that has been described by the PA profession as similar to drinking water out of a fire hydrant. The intensity of this rigorous medical education program taxes students to their limits, yet little has been previously described about the student perspective of the experience; the "voice” of the student had not been explored. While PA program faculty members observe the effects of this intensive process on student mood and morale in their daily interactions with students, little other data exists other than these anecdotal observations. There has been a significant deficit in our understanding of the lived experience of the PA student. This study sought to illuminate critical themes regarding this educational process and its effect on students with the goal of identifying opportunities for programmatic improvements and interventions designed to ameliorate student challenges while maintaining the educational rigor necessary to develop safe and effective health care providers.

Data were collected via two audio recorded focus group interviews with 10 volunteer student subjects, each lasting approximately 90 minutes. The initial focus group session utilized IRB approved script prompts which included questions on the students' experience of the year overall and whether their experience changed over time, on the experience of being a member of a cohort, and about the mood and morale of individuals 
and the group during the didactic phase. The second focus group discussion incorporated questions derived from themes identified from responses by participants during the first session, including the emotional aspects of the educational process, the experience of feeling incompetent, the loss of confidence, the development of a new professional identity, and the role of the cohort in individual and group successes. At the conclusion of the focus group sessions, the digital recordings were professionally transcribed and subjected to a process of conventional content analysis. Individual meaning units, or quotations, were grouped into categories with content commonalities in order to identify themes across the two sessions. As this research is based on qualitative data, the findings elaborated in this chapter include a discussion of the results.

\section{Content Analysis Themes and Subthemes}

During the two focus group sessions, study participants shared their personal experiences as well as their experiences as members of the class cohort during the didactic phase of their PA education program. While individuals each had their own unique perspectives on the events of that year, there was a clear consensus among the group members that their collective journey had a beginning, a middle and an end. This three-stage process is the overarching theme of this medical education experience. To share the voice of the students during their didactic year at the study institution, I begin by describing the data regarding the study participant experience of the year overall and then tie their commentary back to the theoretical frameworks of the human change models introduced in chapter 2 [i.e., Seashore (1975), Menninger (1988), Bridges (1991), Schlossberg et al. (1995), and others]. 
Within the context of this three-stage lived educational process, study participants shared a number of emotional facets of their student experience during the didactic year. The emotional nature of this educational process will be discussed within the context of each of the segments of the journey — the beginning, the middle and the end. While each stage had its own unique emotional issues, some emotions recurred in patterns throughout the year. In this chapter I present and discuss the overarching theme and the emotional subthemes that emerged from the content analysis utilizing the following organizational structure:

The Experience of the Year Overall: A Three-Stage Process

Transition, Change and Transformation

The Beginning: "I was ecstatic . . . at first!"

Isolation

Guilt

Loss of Confidence and Competence

Subjective Incompetence and Demoralization

Burnout

The Middle: "It was overwhelming”

The Ending: "A light at the end of the tunnel”

Being Out-of-Step: The Outliers

On Becoming: Assuming a New Professional Identity

Finally ... Accomplishment!

Surviving the Process: What Helped

Moving Out and Moving On 
Finally, I conclude the chapter with a summary examination of the data and a discussion of a transformative experience of change in a professional medical education program.

\section{The Experience of the Year Overall}

The admissions process for entry into a PA program, analogous to the application process for medical school, is challenging and competitive. Each year the study program averages between 1,000 and 1,500 applicants for a class of 38-42 students. After matriculation during the summer term, students complete four consecutive academic quarters in a classroom setting before transitioning into the apprenticeship-style clinical phase of the program. This focus group study concentrated on the student experience during the initial didactic phase of the program.

While the conversation among focus group participants included reflections over the entire didactic year, the experience of finding out they had been accepted into the program was identified as a key moment associated with the beginning of their educational journey.

I was ecstatic!

I was actually in shock. I don't think I really fully understood that I actually got in for a couple days.

... you remember exactly where you were in important parts of your life and I remember exactly where I was when I got the call ... (that I got in).

Elation and excitement were common shared memories among student participants — excited for what was ahead and relief that the immense task of going through the application process had ended. The initial elation and excitement of 
acceptance tempered as students matriculated into the program and uncertainty became a more prominent sentiment.

... you apply for a program and you .... . want that to be your career for the rest of your life. But how do you know until you're deep into it and committed? You're taking a leap of faith and you just hope that you like it but you're not sure that you will. I remember being really nervous about that.

I was consumed with fear. Once school started, what will happen if I fail? I've put all this money into it . . . it's been a constant total worry.

After beginning the work of the challenging curriculum, the "newness" of the experience seemed to wear off as the workload increased.

... I can definitely remember the beginning of the year when everyone was just giddy and excited about being in school and totally happy to be here .... then reality set in.

... at some point (the excitement) started to level off . . . and things got harder and harder.

After settling into the routine of the first term, a number of participants mentioned that they felt like they had a "handle" on program expectations and could stay "on top" of the workload. Moving into fall term, however, these sentiments changed for some as students tackled the new and ongoing challenges in the curriculum. Completed introductory courses were replaced with new and increasingly difficult coursework in medicine; student workloads increased in quantity and complexity.

It was like I had a groove in the summertime. I was busy, but I could go out every Friday. Whereas, in the fall and the winter, it was just like ... ugh.

I thought it was really hard at first and then by the end of that summer term I had gotten on top of it. Fall term all of a sudden everything is really, really hard again. I remember being completely upset with the fact that I was doing well in the summer and now all of a sudden I am struggling to stay alive.

I started off thinking “'I got this” . . . and then fall quarter hit . . . 
The majority of study participants identified fall and winter as the most difficult and overwhelming terms during their didactic experience.

It can't get worse than this. And then it does! And you're like . . . "Okay, well this is obviously the worst.” And you go a little bit further and it gets worse again! And so you just have to quit telling yourself, "This is as bad as it can get." Because then it just does (get worse).

It was kind of like wave after wave. Something little would happen and then you'd never really get a chance to recover from that. There is a cumulative effect of getting behind and ... you never had time to really regroup.

I think January was my worst time because we'd come back from break . . . it was a really busy month and I just didn't deal very well with the weather and having to sit in class.

So, yeah, I had trouble with January, February, right in there.

These reflections are illustrative of the metaphorical comparisons between the pace of PA education and the experience of drinking water from a fire hydrant. The relentlessness and intensity of the process creates an overwhelming sense that not only is this a difficult journey, but a never-ending one as well. At this point in the didactic year students are too distant from the beginning of the year to remember the euphoria and excitement of their admittance and similarly too remote from completing the year to be able to see the light at the end of the tunnel. The midpoint of the year, encompassing the majority of the fall and winter terms, was clearly identified as the most difficult time period of the first year classroom experience.

As the didactic year progressed, students entered the spring term with nine months of accumulated educational experiences under their belts. The general tone of the commentary now reflects a more settled and positive approach as they neared the end of the didactic phase of their education. 
I feel like I'm more efficient at my studying. I've kinda figured out how to do it. Even though there is still tons to do, I can get it done quicker.

Well, I never thought I would say it, but I think I found my groove in PA school finally (after all this time).

I feel like (class) morale kind of came up towards the end and we were kind of all together on the same page. Definitely a weird evolution to the whole morale and momentum and motivation throughout each quarter I thought.

One student summed up their overall experience of the didactic year with this description:

Morale was high in the summer and then around fall it started to drop and then winter it really, really just dropped precipitously and then around May it started to come up again.

As the conversation shifted to the end of the didactic phase and the transition into the clinical phase of the educational journey, several participants recalled increasing anxiety about their perceived preparation (or lack thereof) for the upcoming new experience.

How did I feel (about leaving the classroom)? Terrified!

I ditto that!

Me, too.

Okay ... before the first (clinical) rotation I was freaking out. In my head I knew how to do classwork, I knew how to take tests, you know? Just sit all day (in class) and study all night. I can do that. But this whole new transition to working with docs and (patients) and being (competent)? I was just freaking out not knowing whether I could really do it.

There's something familiar about being a student. You know how to do it; you are great at it and then pretty soon it comes to an end and now you are going to be on your own. That's another big transforming event. 
Study participant descriptions of their didactic year experiences overall are thematically reminiscent of the three-stage models of change discussed earlier, and worthy of additional discussion.

\section{Transition, Change and Transformation: A Three-Stage Process With Emotional Implications}

The transformative process to become a PA involves mastery of a huge body of complex medical knowledge as well as a profound role transition from one perspective to another-a professional development journey that is both phenomenologically and psychologically challenging. The journey described by focus group participants began with excitement and euphoria upon matriculation, followed by a rapid emotional decline once the true work began in earnest. The "bottoming out" of morale and loss of enthusiasm seemed to occur for most students during the middle portion of the didactic year (fall and winter terms in the study program) after which morale picked up as the end of the didactic year approaches and students eagerly anticipate moving on to the next phase of their education. Then, as the transition to the clinical phase of the educational process became a reality, anxiety becomes more prominent. This pattern mirrors the three-stage models of change depicted by each of the theoretical frameworks of human change discussed in chapter 2. Seashore’s (1975) “mini-life cycle” (p. 2) of professional development programs identifies predictable patterns of group morale changes that can be observed to occur over a period of time. The morale changes, as described by the Menninger (1975) Morale Curve, are initially characterized by high morale upon entrance into a new program followed by a quick downturn in morale as students are challenged by the reality of loss (Crisis of Arrival; Menninger 1975). Loss of old competencies, 
identities, routines and relationships all contribute to a deepening loss of morale as students enter the Crisis of Engagement (Menninger, 1975), where the unrealistically positive expectations held at the beginning of the students’ professional program are replaced with the realities of the workload and the challenges required to successfully meet programmatic expectations. As time goes on, students develop a "new sense of self" (Menninger, 1988, p. 208) as they acclimate to the tasks and develop improved mastery of content knowledge and skills. Menninger’s Crisis of Acceptance coincides with improved efficiencies and an increased sense of competence along with an upturn in morale as excitement builds toward the culmination of the didactic year. Finally, students experience the Crisis of Re-Entry (Menninger, 1975) as the end of the year brings the reality of the upcoming transition to clinical phase to the forefront. While Menninger described this step as its own unique transitional phase, it appears to occur in concert with the Crisis of Acceptance where student excitement about finishing a known component of the program is infused with increasing anxiety about the unknown challenges that lie ahead. Thus begins the morale curve cycle once again.

Just as Menninger (1975) described predictable patterns of morale among Peace Corps volunteers during their international service, a similar pattern of adjustment was depicted by Lysgaard (1955) in the cross-cultural literature. Elation upon arrival to a new cultural setting followed by a crisis in adjustment that included confusion and depression was coined “culture shock” (Gullahorn \& Gullahorn, 1963, p. 49), a condition that improves only as sojourners master the attitudes, knowledge and skills necessary for adaptation into the culture. Bridges’ (2003) model of transitions, also described the experience of the human change process as a three-stage model beginning with the 
experience of loss. “Ending, Losing and Letting Go” (Bridges, 2003, p. 8) of the old way of being is followed by “The Neutral Zone” (Bridges, 2003, p. 8)—a psychological “noman's land” (Bridges, 2003, p. 8) where the individual is no longer who they were but are not yet who they are going to be. This middle phase leads to “The New Beginning” (Bridges, 2003, p. 8) where the individual assumes their new role. Van Gennep’s (1960) Rites of Passage described ceremonial transitions or passages from one generational or role status to another. Individuals move out of familiar roles through rites of separation (pre-liminary) into a liminal status (liminary) — a transitional state that is between what was and what is becoming. As the new role or status is attained, individuals are now welcomed to that new role through rites of aggregation or reincorporation into a postliminary state. Similarly, Sargent and Schlossberg (1988) described “Moving Out,” “Moving Through,” and “Moving In,” and Lewin’s (1947) force-field analysis of human change has identified the stages of "unfreezing-change-freezing.” Each of these models describes a process of human development that includes a beginning, a middle and an end; there appears to be a great deal of consistency across several diverse disciplines of study regarding this three-stage approach to human transition and change. Study participants corroborate their experience of the didactic year as having three recognizable stages and the human change models provide theoretical support for anecdotal faculty observations of PA student cohorts as they move through their didactic medical education process in the study program.

In addition to the overarching theme of the three-stage experience, study participants also described emotional features that set apart each of the described stages of the didactic year. In the following sections, each of the three stages-the beginning, 
the middle and the end-are individually discussed in detail in concert with their emotional subthemes.

\section{The Beginning: “I Was Ecstatic ... At First”}

Admission into a highly sought after graduate medical education program requires years of focused effort and an ongoing record of academic and professional successes. The challenges are many and not all applicants are successful. For those who do succeed in being offered a seat in a class, being chosen represents an external acknowledgement of worthiness and readiness. Already described was the jubilation and excitement experienced by matriculants who had successfully survived this highly competitive process designed to identify individuals best suited to a career as a PA-emotions consistent with Menninger’s (1988) Crisis of Arrival. Psychologist Noel Burch (1970) outlined four stages of skill development in his Conscious Competence Learning Model, the first of which applies to novice learners. PA students arrive to their new educational endeavor both excited and simultaneously unaware of how little they know-a stage which Burch described as Unconsciously Incompetent. Excitement and elation are shortlived once students begin to realize the realities of the hard work they were now committed to undertaking, heralding entry into Burch’s Consciously Incompetence stage. Here students begin to recognize the breadth and depth of what it is that they do not know - a sudden awareness of how much there is to learn and a dawning recognition of their deficits. This growing awareness of incompetence is unsettling for adults who were formerly competent in their old roles. Being forced to let go of old competencies is often met with emotional upheaval, an initial phase of transition described by Bridges (1991) as “Ending, Losing, and Letting Go.” This entrance into a transitional process is beset with 
emotions including fear, denial, anger and disorientation, emotions that Bridges attributed to the letting go of the old and acknowledgement of that loss before one can move on to the new.

While an equilibration of admission euphoria is to be expected as students settle into the work of the program, study group participants described not only the loss of excitement and euphoria but also the development of significant sense of uncertainty not long after matriculation. Many voiced a growing insecurity that they had somehow been admitted to the program in error and were not cut out to be successful in this educational endeavor.

I think for me it started that first week when everyone was introducing themselves and telling what their (educational and professional) experiences were. I was sitting there comparing mine to everyone else’s like, "Oh, my god!”

Yeah, I felt totally unprepared. You just start comparing yourself (to others) and you're like, "How did I get in here? They (the program) totally made a mistake!”

The term imposter phenomenon (also known as the imposter syndrome) was originally described by Clance and Imes in a 1978 study of high achieving women. These women did not experience an internal sense of success despite having achieving advanced degrees and functioning successfully as respected professionals in their fields. They attributed their success to luck or other external factors, had doubts about their abilities and were consumed by feelings of inadequacy. While originally thought to be primarily a female personality construct, Cozzarelli and Major (1990) dispelled the gender stereotype and identified imposter phenomenon (IP) in men as well. Clance and Imes' (1978) original research described students who “often fantasize that they were mistakenly admitted to graduate school because of an error by the admissions committee” 
(p. 1), or that “my stupidity will be discovered” (p. 4). These statements are strikingly similar to those shared among study participants who indicate the challenges associated with their experience of imposter phenomenon.

I don’t think I have gotten over that and I’m still dealing with it every day. I don't like having to do things that I'm not good at-and that's ALL of PA school. I haven't made much progress in getting over feeling like an imposter. It has been one of the more significant emotional challenges with PA school.

The pervasive sense of inadequacy or unworthiness associated with imposter syndrome has the potential for psychological implications for those students who suffer from it. Chrisman, Pieper, Clance, Holland, and Glickauf-Hughes (1995) found a link between IP and high rates of depression and anxiety, raising concerns for the psychological adjustment of students as they move into the challenging portions of the curriculum. This relationship was further confirmed by Henning et al. (1998) in their investigation into psychological distress in medical, dental, nursing and pharmacy students. They found a strong relationship between perfectionism, the imposter phenomenon and the development of psychological distress during medical training. Hewitt and Flett (1991) described a perfectionism construct in which perfectionistic individuals engage in "setting unrealistic standards and striving to attain these standards, selective attention to and overgeneralization of failure, stringent self-evaluations, and a tendency to engage in all-or-none thinking whereby only total success or total failure exist as outcomes” (p. 456). Students who tend toward perfectionism might be at greater risk for emotional challenges and may isolate themselves from others. A study of imposter phenomenon among practicing PAs (Prata \& Gietzen, 2007) identified participants who indicated they felt like they were "masquerading" as health care 
professionals; these individuals also suffered from professional isolation due to their attempts to hide self-perceived inadequacies. A larger follow up study of PA graduates (Mattie, Gietzen, Davis, \& Prata, 2008) identified a relationship between imposter syndrome and major depressive disorder, although cause-and-effect was not determined. The interrelatedness of imposter syndrome and psychological distress along with the prominence of this phenomenon within the context of the focus group discussion suggests a need to recognize students who may experience imposter phenomenon in order to provide additional support during their challenging educational program.

In addition to imposter syndrome, the initial downturn in emotions in the beginning phase of the program is also common to the phenomenon of culture shock (Gullahorn \& Gullahorn, 1963). Elation upon arrival to a new cultural setting (medicine) followed by a crisis in adjustment that includes confusion and depression is a common experience for those who leave familiar environs for the unfamiliar and the unknown. This “anxiety that results from losing all one’s familiar cues” (Menninger, 1988, p. 207) is how study participants described their experience of the didactic year.

I remember when I studied abroad (as an undergraduate student), they talked to us in the beginning . . . “The first few weeks you're just gonna be stunned. You're gonna be excited that you're there and being and trying something new, and then over time it's (the excitement) gonna decrease. You're gonna get really hard on yourself and it's gonna be extremely challenging. At that point you get somewhat homesick and then with time it kinda gradually (gets better) and you kinda feel more assimilated into the culture part of it.” I kinda see that with PA schoolinitially it was like a honeymoon start. Summertime was so exciting that we're here and starting it. Winter/spring was just extremely challenging, just trying to get through it all.

I think it is almost like going to a different culture . . . . things being really fun because everything's new at first and then . . . you do it for a month or two and then you're like “Wow, this is still really hard and I'm still not very good at this." 
Just as travelers experience culture shock when journeying to foreign lands with unfamiliar customs and languages, so do students who enter a medical training program without a well-developed understanding of the medical lexicon. A number of participant comments highlighted the language of medicine as being one of the most significant learning challenges of the early didactic phase. Medical terminology is a unique language based on Greek and Latin prefixes, suffixes and word roots relating to medicine and the human body. Whereas ancient languages used to be well incorporated into liberal education in the United States, it is uncommon for present day students to have had robust exposure to Latin prior to entry into graduate level medical education programs. Even students who have amassed significant experience in medical settings prior to matriculation can be overwhelmed by the sheer volume of language acquisition as they move through the medical educational process.

... it's like ... you are learning a whole new culture and you're learning a whole new language.

... when you're dropped into it . . . you don't know the language, and you feel very overwhelmed.

Not only are students learning a vast and complex database of scientific and medical topics, but they are doing so while simultaneously learning a new language.

The beginning phase of the PA education journey, then, is experienced by study participants as initially a euphoric, exhilarating and celebratory time, quickly followed by the loss of excitement and the development of trepidation and unease. The sense of insecurity, of feeling out of place, and of losing one's competencies all contribute to a deepening downturn in group morale, and many study participants describe entering a 
period of feeling overwhelmed, emotionally and educationally. This represents entrance into the middle phase of this transformative educational experience.

\section{The Middle: “It Was Overwhelming”}

As the workload increased and students became fully immersed in the "fire hydrant” experience of PA education, a number of challenges associated with the loss of old familiar routines and competencies were experienced. The euphoria and excitement reported on matriculation quickly gave way to a downturn in emotions leading to the middle portion of the three-stage process that spans nearly half of the didactic year, according to the study participants. Beginning in late summer/early fall and continuing through to the late winter/early spring, the middle stage represents a challenging mélange of emotions. For the majority of the study participants, it was a time of intensity combined with drudgery—constant academic effort to master a never-ending supply of new medical information. Students were too removed from matriculation to remember the euphoria of being accepted, and too far from the end of the didactic year to see the light at the end of the tunnel. In fact for some participants, any reference to the proverbial light at the end of the tunnel during this time of year was perceived somewhat jokingly as an oncoming train heralding the next looming academic or personal disaster.

The middle stage as described by Menninger’s (1975) Crisis of Engagement is characterized by a sense of loss heightened by recognition of the magnitude of the disruption in participant lives. Participants now fully recognize their losses, both real and fantasized. Unrealistic expectations about the expected difficulty level of the workload or its effects on one’s personal life are now gone. Kubler-Ross (1969) described this experience of change as an emotional loss intertwined with stages of grieving, beginning 
with denial then working through anger, bargaining, depression and acceptance. The middle stage of the didactic year of PA education is particularly characterized by anger and frustration upon realization of these losses, potentially resulting in depressed mood. Study participants noted a continuing decline in mood and morale the further they moved from matriculation. The emotional nature of this middle stage of PA education includes a sense of isolation, guilt, loss of confidence, loss of competence and the experience of burnout_an “emotional valley” of despair, as described by one individual; a valley that did not improve for many until late in the didactic year.

Many study participants reported changes in their interpersonal relationships while they were in PA school. One of the predominant emotional experiences expressed was a perceived isolation from others coupled with guilt associated with coping with overwhelming classroom demands that regularly preempted time with family and friends. Isolation may be an outcome among practicing PAs with impostor phenomenon, and isolation also appears to be one of the emotional issues experienced by PA students during their didactic education.

\section{Isolation}

The isolation described by study participants was in part a physical isolation, as full time classwork followed by off-hours studying significantly limits time available to spend with loved ones. But the experience of isolation conveyed in the focus group discussion was more than just physical or situational—it also suggests an emotional isolation; a sense that no one could truly understand their experience as a PA student unless it was another PA student, or perhaps another health professions student immersed in a similarly rigorous program. 
The only people I felt really understood us were the PA students in the class ahead of us.

I think some of the med(ical) students understood. I just finished working with one this last rotation and he pitied me (during the didactic year). He was like, "Man, I felt so sorry for you guys."

My wife understood (PA school) was going to be a lot of work but she didn't “get” what we were doing . . . There's no reference for them. They don't really understand it ...

I have given up trying to explain what my daily life is like and I'm not sure I like that. It's too hard to try to give anyone an idea of what it's like in PA school.

Another facet of isolation was related to language. As noted earlier, immersion into the language of medicine is an important part of the cultural assimilation into a new role as a healthcare provider. Once students gain competence in the use of medical terminology, it becomes incorporated into who they are-a part of their new identity is no longer perceived as separate from themselves.

I don't know what language it was but we all spoke the same one. We would go out (socially) and others would hang out with us and all we talked about was (medicine)—not anything anybody else could understand.

... when you start to feel pressure from all of your outside friends to hang out with them, just invite them along to a class happy hour and then they'll never ask you (to hang out) again.

One study participant described sadness that she had lost the ability to talk with her father on an equal level about medical topics. As a science professional himself, he had previously been able to share common experiences during the early part of his daughter's PA education. She mourned the loss of that sharing relationship once she realized that she had surpassed his level of medical knowledge, and described feeling isolated from him as a result. Familiarity with the language of medicine not only provides entrée into the culture of medicine, it also creates separation and isolation from those who 
are not part of the medical culture. Being an “insider” in the family of medicine

reinforces the transformative nature of the experience; as a result of being in this program students are somehow different from others while becoming more like each other.

It's hard for other people even to relate, you know?

It is kind of like an AA (Alcoholics Anonymous) group. You share a common experience that you can’t (explain) to anyone who is outside of the group. You can try and describe it but you can’t really put it into words.

It is sort of an “in-group” or “out-of-the-group” kind of a mentality, I think.

Study participants also perceived the dynamics of their personal relationships as changing during their didactic experience. Just as Seashore (1975) described "feelings of anger, depression, resentment, envy and irrelevance among family and close friends because of (the student's) participation in the program” (p. 1), participants shared similar reactions among their family and friends.

I was not communicating with my family as much. They feel like they are left out sometimes. They don't understand why I don't have as much time for them.

They're upset and there is not much you can do about it.

My mom is afraid to call me. I think my family is just waiting for (the old) me to resurface.

It is very strange going back (home) because most of the guys that I grew up with ... they really don't like the fact that I am going to school.

\section{Guilt}

Intertwined with the sense of isolation from family and friends outside of the PA program was a corresponding sense of guilt.

(I had) this tremendous sense of guilt whenever I was not doing something school related. I had a lot of guilt anyway.

Every time I wasn't doing something ... (for) PA school . . I felt terribly guilty about it. It was hard to enjoy really fun wonderful things because "oh, my-gosh" you shouldn't be doing this. 
Several participants described being proverbially "stuck between a rock and a hard place"-feeling guilty when spending time with family and not concentrating on school, and feeling guilty when focusing on school and not attending to their families.

Family would (want to talk with you) and they would have serious problems and you are like, “Man, I don’t have an hour to spare.” That was hard.

I think I feel more guilt when I am away from school than I do about being away from friends and family. I feel like I'm missing out on these opportunities to further my knowledge and grow and stuff. It's irrational. I understand that. It's impossible to avoid thinking about school though.

In addition to an emotional valley characterized by a sense of loss, isolation and guilt due to the efforts associated with programmatic demands, study participants also described significant internal emotional challenges related to their level of confidence and sense of competence. At matriculation, the spirit of the cohort was positive and confident; being admitted to the class was reinforcement to each student that they had acquired academic and professional skills, knowledge and attitudes worthy of entrance into a medical education program. In concert with the onset of imposter phenomenon in the beginning of the program, however, came also the loss of confidence and the sense that study participants had lost the competencies they had acquired prior to matriculation.

\section{Loss of Confidence and Competence}

A prominent emotional experience of this intensive learning process described by study participants was the loss of confidence that developed as they moved through the didactic curriculum. Several commented on the negative impact that this loss of confidence had on their well-being.

I definitely feel the more you learn the more you realize you don't know-it's terrifying. 
My perception of the year it just seemed to continually get harder and I wasn't sure if it was really getting harder and harder or if I was getting tired. Whenever we got comfortable with something we would be asked to do something a little more complex. You never were able to get comfortable with what you were doing.

It ultimately really made me insecure. Last year I just remember thinking "Is this going to be manageable?" “Am I going to come out of this alive?” I think that insecurity was ... disorienting.

Confidence is defined as "belief in oneself and one's powers or abilities; selfconfidence; self-reliance; assurance” (Dictionary.com, n.d.a, para. 2). Holland, Middleton, and Uys (2012) defined professional confidence as having four components: affect (feelings associated with action), reflection (thoughtfully examining one's actions and intentions), higher cognitive functioning (learning and integration of concepts, decision making, attention, motivation and memory) and finally, action. In addition to describing the requirement for a solid foundation of knowledge, these two definitions also highlight the significant emotional underpinnings of confidence. Study participant discomfort with program activities suggests a relationship between confidence and competence — that the inability to perform comfortably impacts confidence by imparting self-awareness of incompetence.

I think confidence is a huge deal for me. Anything I have ever done I have worked really hard to become competent. I finally just became a competent person at my job that I left (to attend PA school) and I remember the last day of work thinking, "Wow, this is going to be rough. I am walking away from something I am doing well knowing that I am probably not going to be all that great at what I am doing next for a long time.” It was hard.

Yeah, I think the thing that I've been surprised with PA school is that it's totally shaken my whole self-confidence to the core, you know.

Some study participants perceived the loss of confidence as an intentional goal of the program. 
Yeah, I think it was important to be stripped of your confidence at the beginning. By coming in and realizing that you knew absolutely nothing about anything you're way more open to learning.

This is the end of who I was and the beginning of who I will be-a kind of breaking down and rebuilding. I (now) see it is a process of building that back up as you go out and learn to become a professional.

This externalization implies a belief that the educational program had purposefully orchestrated a "breaking down” process before building students back up into their new PA role as opposed to recognition that loss of confidence is an individual response to the need to learn new skills. Bridges’ (2003) model on transitions described this as the "Neutral Zone" (p. 8) in which the old ways of being are not completely gone, yet the new ways have not been fully assimilated. He has characterized this as a psychological “no-man’s land” (p. 8) between the old and new identity, fraught with discomfort and confusion and ultimately where transformation occurs. Although unsettling, "it is the chaos into which the old form dissolves and from which the new form emerges” (p. 9). Van Gennep (1960, p. 11) described this middle stage as liminal, where liminal is defined as a transitional state or threshold. Liminality refers to an individual's transition between the old and new social or cultural status, or between what was and what is becoming.

Because everything that you used to know and be is gone and the things that you're trying to know and trying to be are not quite there yet and so you don't really have anything to grab onto and say, "I am good at this." So for that brief time when you don't know anything . . . you really do feel down and you (wonder) "What is my role?"

While students retain the competencies they acquired in professional roles prior to matriculation, these old competencies are frequently not transferable within the context of a new professional role as a PA. The loss of confidence experienced by study participants 
is not attributable to the loss of old competencies, then, but instead is due to the unsettling experience of being thrust into the novice role of a new profession requiring the development of new competencies.

... the way that (the program) rocked everyone's confidence might have been good because I know I came into the program thinking that I had the answer to everything or that there was one answer to everything. And in medicine there is no one right answer necessarily. It's good to realize what you don't know and how much you don't know.

I think that, while it's necessary for us to lose our confidence in order to learn a new role, it does affect our morale and I think that's one of the biggest reasons we have such a hard time getting through, you know, in good spirits, through some parts of the year.

When I went through the didactic year, having your comfort level challenged (affected) my confidence as a person (and it) just went down over the course of the year.

While many study participants considered this loss of confidence to be morale

shattering, others described it as a positive feature of the didactic phase of their education.

... (a loss of confidence) is a necessary process to go through. You feel like you don't know what you're doing and recognize that you're a blank slate. We can learn new things all together and start to appreciate this new profession that we're getting ourselves into.

Yeah, and to be willing to admit when you don't know things, I think is part of it, too.

... I definitely see that there's a lot of growth happens there.

Competence, as defined by the Oxford Dictionary (n.d.), is "the ability to do something successfully or efficiently” (para. 1). Epstein and Hundert (2002) have defined professional competence in the medical field as "the habitual and judicious use of communication, knowledge, technical skills, clinical reasoning, emotions, values, and reflection in daily practice for the benefit of the individual and community being served” 
(p. 226.) Clearly, the rapidity of the didactic phase of PA education limits the attainment of competence by its pace alone. The curriculum introduces medical topics in an abbreviated and condensed fashion, requiring students to rapidly assimilate foundational information in a brief amount of time, moving on to the next topic before mastery is achieved. Successful completion of a course in medicine does not confer competence, a reality that creates frustration among didactic year students given how hard they work to learn the content. Competence is achieved not through the completion of a course of study alone, but through repeated practice or application of a skill, and the acquisition of competency appears to move along a developmental continuum. Because didactic year students have limited opportunities in which to apply the new knowledge they are assimilating, or practice the beginning skills they are learning, their sense of competence tends to be delayed. In their original research for the Air Force Office of Scientific Research, Dreyfus (2004) outlined a five-stage model of adult skill acquisition through which individuals progress, beginning with a novice stage and moving to advanced beginner, competence, proficiency and ending with expertise. The novice stage involves students learning context-free, rule-based content-in other words, just the facts. According to Dreyfus \& Dreyfus (1980), “the novice pilot knows how to read cockpit instruments and how to manipulate the controls” (p. 7) based on learned rules, but they do not gain competence at flying until they have amassed considerable opportunities to practice application of the principles they have learned. Learning at the novice level tends to be other-directed; students lack the context needed to be self-directed learners, focusing instead on the memorization of facts while relying upon faculty for direction. In addition to lacking contextual foundations for skill development, learning new skills is a 
process fraught with emotion. In an article by Daley (1999, p. 139) comparing novice and expert nurses, novices described the emotional nature of their learning as feeling “overwhelmed,” “scared to death” and being fearful of harming someone. Skovholt and Ronnestad (2003) described the learning challenges of novice learners as stressful and emotionally exhausting, attributing those challenges to the "inherent, but often unknown to the novice, ambiguity of professional work” (p. 46.) PA student anxiety about the unknown challenges that lay ahead is stress inducing, and the amorphous fear of potentially causing harm to patients if medical content is not appropriately mastered amplifies that stress level to an even greater degree. These descriptions of the emotional nature of the novice status are not dissimilar from comments made by PA study participants regarding the middle stage of their didactic education.

\section{Subjective Incompetence and Demoralization}

As study participants struggled to orient themselves to their new role as PA students and future health care providers, they were challenged with the reality of lossloss of established relationships, familiar routines and a diminished sense of competence. Skills and competencies they had mastered prior to matriculation were set aside in order to learn new knowledge, skills and attitudes. What they were once good at no longer mattered as they were all propelled into the role of a novice learner. The negative impact this lack of confidence and sense of incompetence had on their personal outlooks was significant for many. Loss of mastery in adult learners may exemplify “subjective incompetence” where an individual experiences "a state of self-perceived incapacity to 
act at some minimal level according to some internalized standard in a specific stressful situation” (de Figueiredo \& Frank, 1982, p. 353).

de Figueiredo and Frank (1982) went on to identify subjective incompetence as the clinical hallmark of demoralization, the definition of which is "to deprive (a person or persons) of spirit, courage, discipline, etc.; destroy the morale of; to throw a person into disorder or confusion” (Dictionary.com, n.d.b, para. 1). Demoralization, then, occurs when subjective incompetence coexists with distress (de Figueiredo, 1983, 1993). D. Clark and Kissane (2002) described the symptom expression of demoralization as a process in response to a stressor that begins with feelings of apprehension and panic leading to helplessness, feelings of incompetence, impotence, a loss of control over life, hopelessness and feelings of shame and isolation (p. 735). Bandura (1982) described the sense of futility individuals may develop when they perceive themselves to be ineffectual, leading to "self-devaluation and despondency" (p. 140)—a condition not unlike subjective incompetence. In addition to subjective incompetence, Frank (1974) highlighted impairment in coping ability as a key feature of demoralization, with feelings of hopelessness and reduced self-esteem. Failing to meet one’s own internal expectations (or those of others), or having difficulty with coping (D. Clark \& Kissane, 2002) are all phenomena that may exemplify the realization of losses that take place during Menninger's (1988) Crisis of Engagement during this midpoint in the academic phase of PA education. Study participant descriptions of the experience of the middle stage of PA education clearly evoke a downturn in morale in addition to the development of apprehension, panic, a loss of confidence and a sense of isolation. While demoralization develops as a result of psychological distress and can become a psychological stressor in 
and of itself, it has characteristics that distinguish it from clinical depression (D. Clark \& Kissane, 2002; Rickelman, 2002). It may, however, be a precursor to depression (Rickelman, 2002), and could potentially serve as a warning sign in students who are struggling with the challenges of the educational process. Demoralization that continues without respite may lead to emotional exhaustion, or "burnout."

\section{Burnout}

Screw it.

Yeah ....

While initially identified by Freudenberger in 1974, Maslach (1982) has been credited with subsequently describing burnout as a psychological construct in the helping professions. Maslach, Schaufeli, and Leiter (2001) defined it as a syndrome that includes emotional exhaustion, a reduced sense of personal accomplishment and the development of cynicism and depersonalization. Burnout has been identified as an ongoing issue among practicing health care providers and health professions students (Cecil, McHale, Hart, \& Laidlaw, 2014; Shanafelt et al., 2012; Varner \& Foutch, 2014.) An estimated prevalence of $49 \%$ of medical students in the United States experience burnout (IsHak et al., 2013); little is known about this phenomenon in PA students. During the focus group discussions, however, study participants clearly describe features of burnout during the middle stage of their didactic year.

I burned out . . . apathetic towards school and I hated that feeling but it was like "I am just so done” ... Or, “do I even care?”

I feel like we kinda lost it-our coherence, togetherness, in (late winter/early spring). We were all personally really tired so we didn't have the energy to support each other. "I just gotta get through this" and . . . we didn't have the emotional energy to deal with other people's problems. 
I have reached the maximum. I just can’t push myself any further.

I remember one (YouTube video that) kind of sums up what this (felt like)—a guy who said "fuck this shit" and throws all these papers up in the air and storms out. Then in the next picture he is picking them all up saying "Oh wait, it's Thursday and I still need these.” I can’t burn my (class notes) even though I want to.

... you just reach a point where you're just, like, "I can't deal with you-I've got my own personal crap goin’ on. I just can’t. I’m sorry.”

... it just feels like the whole time you're just barely . . . barely making it and if even one little thing goes wrong, it's fucked-it's all off.

One participant voiced his frustration at being unable to offer other people

anything of value; he recognized the help and support he was receiving from others but

felt he had nothing left to give back. Another described being overwhelmed to the point

where poor personal choices seemed like viable survival mechanisms.

We were in a vicious cycle. When I get to burnout I turn to really addictive behaviors and it gets really unhealthy and I would sort of rely on those behaviors to get me through but then I would be too tired and worn out and sick . . . then I couldn't study for my test and then I wouldn't pass the test and I would have to retake the test and there would be this huge vicious cycle.

The fire hydrant experience of the didactic year of PA education can result a sense of isolation, guilt, loss of confidence and the development of subjective incompetence, particularly during the middle stage. For some students, the educational process appears to have the potential to lead to demoralization, and may ultimately result in burnout (Maslach et al., 2001), including emotional exhaustion, a reduced sense of personal accomplishment and the development of cynicism and depersonalization.

While there is a limited understanding of psychological distress among PA students (M. H. Brown, 2004; Childers et al., 2012; Glicken \& Gray, 1993; Kuhn et al., 2005; O’Brien et al., 2012), there is an abundance of literature regarding psychological 
distress among other health professions students as previously noted (Cardall et al., 2008;

D. C. Clark \& Zeldow, 1988; Dickstein et al., 1990; Dyrbye, Thomas, \& Shanafelt, 2006;

Helmers et al., 1997; Roberts, 2010). PA student participants in this study describe similar stressors, and while questions about anxiety and depression were not specifically asked during the focus group sessions, the tenor of the conversations suggests the likelihood that these psychological challenges may exist within this collective group of students. While the vast majority of students respond resiliently to the challenges and manage the stress of PA school effectively and successfully, concern should exist for the student who may be challenged by this stressful experience and may descend into demoralization and depression; the potential risk for suicidality described in other health professions programs cannot be disregarded in this population (Rickelman, 2002;

Schwenk et al., 2010).

\section{The Ending: “A Light at the End of the Tunnel”}

After navigating the lengthy middle portion of the three-stage process that began in late summer/early fall and continued through to the late winter/early spring, study participants describe moving into the ending stage. As they continued along their educational journey, this stage finds participants coming to grips with their new reality while learning how to assess their situation with a more realistic eye, resulting in improved morale. As students continue their adjustment to their new professional culture, a developing sense of satisfaction and personal growth appears—a pattern remarkably similar to Menninger’s (1975) Crisis of Acceptance.

We got kind of beat down throughout the year and I remember the low point around the end of fall or during winter . . . . then it felt like everyone picked up at 
the end of the (academic) year and kind of just barreled through toward the end. It was like we picked up some momentum or just shook it off and got through.

I feel like (class) morale kind of came up towards the end and we were kind of all together on the same page. Definitely a weird evolution to the whole morale momentum throughout each quarter I thought.

... then near the end there was light at the end of the tunnel and there was a lot more motivation to get through your classes.

At this point in the didactic year, references to the light at the end of the tunnel no longer suggest the presence of a perceived threat; instead they now indicate that participants can see the end of the first part of their educational journey. As described by Menninger (1988), participants in this stage have achieved “a new sense of self” (p. 208), with enhanced mastery over their new life circumstances. Study participant comments mirror this improved self-efficacy.

I feel like I'm more efficient at my studying. I've kinda figured out how to do it. Even though there is still tons to do, I can get it done quicker.

Well, I never thought I would say it, but I think I found my groove in PA school finally (after all this time).

Student inquiry skills and self-directed learning abilities have matured significantly at this point in the year. They are increasingly competent at defining clinical questions and locating empiric, evidence-based information with which to drive their medical thinking and decision-making. As they improve their ability to distinguish relevant material from that which is irrelevant, student efficiency in studying improves, resulting in a reduction in time necessary to complete academic tasks. Movement through the curriculum allows mastery of some skills, transforming students from the consciously incompetent stage to the consciously competent stage (Burch, 1970). Dreyfus (2004) described the transition from the novice stage to that of advanced beginner as one where 
increasingly acceptable skills are demonstrated. Physical examination techniques, for example, were once laboriously performed by novice PA students in a step-by-step memorized manner without a clear understanding of the implications of their results. During this ending stage of the didactic year, students are now performing skills that have become so practiced as to be second nature; they are now beginning to distinguish normal from abnormal results, signaling the advancement of their new identity as a health care provider. Ultimately, according to Bridges (2003), individuals emerge from the "Neutral Zone” into the "New Beginning” (p. 57) where this new sense of identity is developed. At this point, the individuals are ready to move forward in their new role with improved clarity and confidence. Van Gennep (1960) described the individual as being reincorporated into a new social or occupational group as the new role or status is assumed. Having mastered the role of classroom student, study participants excitedly anticipate leaving their didactic year behind for the hands-on, patient care role during the second year of the program.

As the end of the didactic year approaches, plans for the upcoming transition to the clinical phase of the program now serve as a powerful motivator for students to redouble their efforts toward continuing their educational quest. Anticipation about the culmination of the didactic year and the process of moving on to the exhilarating handson clinical phase of their medical education experience, however, finds excited study participants developing a sense of unease. They describe another fluctuation in morale in keeping with Menninger’s (1975) Crisis of Re-Entry. They now have mixed feelings about leaving the environment to which they had become acclimated; departure from the 
safety of classroom routines for the unknown aspects of the clinical phase reactivated the anxiety associated with the Crisis of Arrival (Menninger 1975).

Okay ... before the first (clinical) rotation I was freaking out. In my head I knew how to do classwork, I knew how to take tests, you know? Just sit all day (in class) and study all night. I can do that. But this whole new transition to working with docs and (patients) and being (competent)? I was just freaking out not knowing whether I could really do it.

I worry that they (clinical preceptors) are going to expect me to be smarter than I am or know more than I do .... I think for me that's where most of the anxiety comes from-not knowing what is going to be expected of me and how my abilities are going to relate to that.

It's like I wish I was better at this point and I don't know how to do that.

These comments are reminiscent of sentiments expressed during the beginning phase of the educational experience related to Imposter Phenomenon (Clance \& Imes, 1978). Despite their many successes with the didactic PA curriculum over the course of the year, study participants once again doubt their abilities and question their capacity to perform to the level expected in the next phase of their education. Concerns that they remembered little from a year dedicated to intensive study are prominent: “Oh, my-gosh, [they] are going to expect me to know everything and I know nothing.”

The ending phase, then, is characterized by a gradual upswing in emotions and morale as new professional competencies are acquired and inquiry skills are improved. Study participants have assimilated the language of medicine and are beginning to develop their role identity as a PA. They fully recognize they have not achieved their final goal yet, as evidenced by the expressed anxiety regarding the beginning of the clinical (or the next novice) phase of their education, but they see the path they are on and the goal is ever closer. 
The didactic year of PA education represents a significant period of transition, change and transformation—-both personal as well as professional. Study participant descriptions of their didactic year experiences overall are evocative of the three-stage models of human change and transformation. While students shared a common educational experience, not all individual participants experienced the educational process in the same way. In the next section, I describe the focus group narratives of those whose experience did not mirror that of their cohort colleagues.

\section{Out-of-Step: The Outliers}

While study group participants generally agreed that the emotional roller coaster ride experienced during their didactic education followed the three-stage pattern described, some also expressed periods of being “off-cycle” or "out-of-step” from their class colleagues. Outliers, those whose emotional states did not match the cohort as a whole at any given time, attributed their emotional mismatches to personal challenges emanating from life experiences outside of the program.

... the times that my morale was the opposite of the class were times when I was having personal issues and didn't know what to do or who to talk to . . times that I felt like the class was still buzzing along up here (high) and I was down low.

All of us kind of experienced it differently. For some people fall or winter term was the hardest and for others (it was just based on) what was going on in their lives at the time.

I think there's definitely a mood [morale] of the group as a whole. When I was having a dip, [the class morale] was really helpful and it brought me right back up. It wasn't acceptable to get too discouraged because there were other people around you who had good attitudes and got you back on board.

. . . the class morale could be kind of (a) steady state. Then you could fluctuate above or below depending on how you were doing as the year went by. 
Students expressing a sense of being out-of-step with the group may be describing the interplay between morale, a measure of group esprit de corps, and mood, which is more an individual measure of emotional state. Illness, family emergencies, financial worries, conflicts in interpersonal relationships, parenting challenges-all occur at various times in students' lives without regard to the timing of the overarching stressors of the program itself. During times in which students' personal lives are stable, the three-stage morale curve of the cohort may be the preeminent experience, whereas during times of interpersonal instability personal challenges may take precedence-resulting in students feeling out-of-step emotionally from their colleagues. Students who experience interpersonal challenges during this intensive educational process may carry a greater psychological burden and may be at a higher risk for demoralization, depression or other manifestations of psychological distress. Of particular concern may be those students who experience mood challenges during times when cohort morale is simultaneously at its low point. There is a need for additional exploration of the interplay between mood and morale during intensive medical education programs in order to identify opportunities for improved support strategies to assist students through these challenging and transformational times.

\section{On Becoming: Assuming a New Professional Identity}

Medical education is a process by which specialized content knowledge is transmitted from current members of the medical culture to novices seeking entrance into that medical culture. This process entails much more than just the transfer of knowledge; it also promotes enculturation into a profession by socializing norms and behaviors, thus transforming students into medical professionals (Lave, 1993). Cleland and Johnston 
(2012) have described medical education as a "prolonged developmental process . . . that appears to operate on a crudely 'passive to active’ continuum” (p. 836). Jarvis-Selinger, Pratt, and Regehr (2012) depicted the formation of identity among medical trainees as an adaptive, developmental process that happens simultaneously at two levels: (1) at the level of the individual, which involves the psychological development of the person and (2) at the collective level, which involves a socialization of the person into appropriate roles and forms of participation in the community's work. (pp. 1-2)

These points of view from the two professions of psychology and sociology distinguish between the concept of self-identity as development of individual psychological factors and the concept of role identity as role development that is socially constructed (Roeser et al., 2006). Students engaged in a PA education program spend intense amounts of time focusing on the knowledge acquisition aspect of the professional development process and may not recognize the dramatic impact such programs have on their personal identity until they are well into their course of study. Reflecting back over their didactic year, participants in this research study described a transformative aspect to their emerging identities as PAs.

My goals and priorities for my life or PA school were a little bit different (before I started PA school) than they are now. I am still trying to figure out what I think is most important and what the future looks like.

I think my world view has changed and the foundations that I had before have definitely shifted in some aspects.

I guess I feel more one dimensional now. (Medicine) kind of consumes me. There's an element of losing things that I used to identify with before but I don't identify with any more.

Looking back, I have changed a lot after my first year. I am not as engaging anymore. I have lost something (in the process). 
If the process of "becoming” a member of a new profession is indeed developmental, it follows that PA students will not have completed their transformational journey just one year into their educational experience. Chickering (1969) identified seven vectors or dimensions of identify formation, including the development of competence, management of emotions, development of autonomy, establishment of identity, freeing of interpersonal relationships, developing purpose and developing integrity. While all elements of Chickering's seven dimensions can be seen in the narratives of the focus group transcripts, the work of first year students seems centered upon the vectors of competence, emotion and establishment of identity. At the end of their didactic year, having traveled through the three distinctly identified stages of their educational journey, these first year students are not yet finished products. They have mastered the role of successful PA student in the classroom setting, but have not achieved the proficiency level of a professional PA—-thus remaining in a liminal state ala Van Gennep (1960), or a Neutral Zone ala Bridges (1985). Comments from study participants emphasize the impact that the program had upon the liminality of their identity as individuals.

I do think the program has changed me but I'm not sure how yet and I don't know where I am going-but I will figure it out.

I have rounded the corner and I get glimpses of the person that I am going to be ... or maybe I had lost track of who I was and I am becoming more comfortable with who I really am-or something to that effect.

After this whole process is over, I guess I am not sure who I am going to be. I feel like I have to go back to all the things I used to like and decide if I still like those things.

I am still learning to deal with who I am. 
The emergence of identity as an evolutionary progression through which the self is continually revised suggests a constructive-developmental perspective of personal growth by which the individual builds new meaning upon past understanding. Erikson (1956) described identity as "a gradual unfolding of the personality through phasespecific psychosocial crises” (p. 128), and “each successive step . . . is a potential crisis because of a radical change in perspective” (Erikson, 1980, p. 57). This reference to crises and perspective change reflects an important connection to transformative learning. Mezirow (1997) described transformative learning as a 10-step process that affects a change in an individual's frame of reference. This process includes cognitive and emotional components, and features a disorienting dilemma commencing the process. The beginning stage of the didactic year of PA education, as identified by study participants, contains this disorienting dilemma-complete with confusion, emotional distress and the experience of Imposter Phenomenon. The middle stage of the didactic year includes Mezirow’s stages of self-examination, critical assessment and feelings of alienation and discontent. The ending stage encompasses Mezirow’s exploration of new ways of acting, building competence and self-confidence in new roles, implementing a new course of action and reintegration with a new perspective. Key features of the didactic year three-stage experience described by study participants are closely aligned with Mezirow’s overview of transformative learning, with the theme of transformation being a significant element of the experience.

I definitely think of the first year (of PA school) as a period of being pretty vulnerable in a lot of aspects of my life-emotionally, intellectually just (everything). I now appreciate how that's the kind of the right mix to totally transform you. 
Obviously (I am entering) a new career which is really exciting but I am not the same person I was when I came in. Fundamentally, yeah, but . . . . what's my life going to look like? I feel like I am in this in between place waiting for that to happen so I can figure it out.

It feels like you are in between two lives.

This is the end of who I was and the beginning of who I will be.

Mezirow’s (1997) trying on of new roles is also a key element of the study

participant experience of the didactic year.

Because everything that you used to know and be is gone and the things that you're trying to know and trying to be are not quite there yet and so you don't really have anything to grab onto and say, "I am good at this." So for that brief time when you don't know anything ... you really do feel down and you (wonder) "What is my role?” But then you get through it and I'm starting to realize ... this is my role and and I can do this.

Study participants describe the transition to their new role as a PA in ways that

suggest two distinct features, the first of which is the expressed sense, "I feel different."

Becoming a professional and having to really increase consciousness about doing things well and being responsible for things (means) I'm no longer in that carefree spontaneous place with people. I am not really sad about that-I'm really happy to be where I am now, but it feels different and it has created a distance in some of (my personal) relationships.

I'll say something (like), "That person looks like they have chronic obstructive pulmonary disease," and (friends will say) "What? Why are you doing that? Why are you talking that way?” I can’t help it-it’s how I think now.

I look at people differently now. I haven't changed (to the point) where I'm changing my friends, but I'm definitely talking to them differently regarding health.

Not only do study participants "feel” different, they also describe other people as now treating them differently.

I definitely find that people are calling me more about medical issues. Like my dad is going through health stuff right now and he's calling me every day going, 
"What should I do? What should I do?” You know, a year or two ago he wouldn't have done that.

I think some people really want to pick your brain for their own health reasons and some people don't wanna talk to you at all about their health problems. In some ways people censor themselves around you or really open themselves up in ways you wouldn't expect based on how the relationship used to be.

The didactic year of PA education represents an intense immersive experience designed to prepare future PAs for the responsibility of caring for patients safely and effectively. The medical education experience is exhausting and challenging, but the change process also results an immense sense of accomplishment among students as they achieve each of the milestones of the program. The next section highlights the study participants’ experiences related to their accomplishments.

\section{Finally ... Accomplishment!}

Looking back on their didactic year of PA education as a whole allowed study participants to sum up their experience, and their comments reflect a sense of wonder about the amount of information they had encountered within the space of a single year.

Yeah, the thing that I've gotten out of PA school is how amazing our ability is to learn. When we started, we knew so little about medicine. And even those of us who had been in the medical field before in various components had no idea (how little we knew).

I feel like PA school is the door to learning medicine and (and I now know) that learning doesn't stop at the end of PA school.

I can't believe how much knowledge was thrown at us and the little bit that I did absorb feels great. I wish I had absorbed more.

While clearly aware of the limitations of their knowledge at this stage in their education (having completed the didactic year but still engaged in the clinical year), study 
participants expressed pride in accomplishment for having successfully completed the didactic phase.

I think anytime you do something really difficult it generates pride. Internally we all feel proud for completing (this year).

It will be probably be one of the biggest accomplishments in my lifetime.

I am ... proud of what we did. I will take that with me and it will be something I can be proud of for a long time. Sometimes it was really hard and sometimes it was miserable but in the end not everybody can do it. Not everybody has the opportunity to do it! I think we are pretty lucky that we got to (go through this process), as miserable as it was at times.

One woman even described her experience of the didactic year as similar to childbirth.

... and I remember having the baby and that it was the worst pain I could possibly imagine. Afterward it wasn't really that big a deal and I probably could do that again. That's kind of how the year felt ... like childbirth.

Despite the references to painful nature of the transformative process (including the notion of this being a process of birthing), comments from the study participant group reflect a satisfaction with the experience and their successful accomplishments during the didactic year. The conversation turned to shared examples of how they "survived"-and those things they considered the most helpful in getting them through a long and difficult year.

\section{Surviving the Process: What Helped}

As they reflected back over the experience of the didactic year, study participants described a variety of phenomena to which they attributed their educational and personal successes. After sorting through the data during the content analysis, three thematic areas emerged: the important role of the cohort, the lack of competition, and programmatic reassurance. 
A cohort is defined as "a group of individuals having a statistical factor (such as age or class membership) in common in a demographic study; a companion or colleague” (Merriam-Webster, n.d.a, para. 1 \& 2). The shared didactic education experience among first year PA student colleagues creates a cohesiveness based on that communal experience, and the relationships formed within the cohort during that experience can provide emotional and educational support.

We don't know each other until we get here and are all thrown into this new environment together. Getting through it all as a team with everyone going in the same direction-everybody knew what you were going through because they were going through it too. I will look back on this experience as something that doesn't happen every day and maybe just once in our lifetime. It was a shared moment between all of us.

It's easy to think about all the horrible parts in terms of studying and the challenges and stuff but one thing that I think we recognize is how close we all got. I think we truly developed a family with classmates and we had a lot of really cool experiences throughout the year.

I did the Peace Corp and that was the same exact experience- - there were 60 of us and we all landed in rural Africa together and there's no option but to bond and become like family. I felt like that with PA school—when we walked through these doors ... our whole class came together pretty well.

I couldn't have finished this year without my study group or people that I like to do activities with. To have people who are in the same boat dealing with this together was key. I don't think I would have been able to get through if I had to do this by myself.

The shared nature of the experience united the class into a cohesive group and provided support-both during class as well as outside of class. Several study participants attributed the cohesive nature of the cohort to a lack of competition within the group. In the study program, once matriculated, students no longer need to compete for status within the class as success is measured by successful passage of coursework and not by class or grade ranking. Students are encouraged to learn by, with and from 
each other and to utilize each class member's unique skills and attributes whenever possible to ensure the success of the entire cohort. Because students have spent their prior academic lives competing in order to achieve their goal of being accepted into a PA program, many have difficulty giving up their competitive nature. Over time, however, the realization that competition does not help them succeed in this new medical education endeavor allows a collaborative relationship to develop within the cohort.

... about four weeks into summer term I realized that it is ridiculous to care about (competition)—we were a team rather than competing against each other.

It was all about sharing and helping each other. There was never a competition . . . A shared experience, a common goal, a noncompetitive environment and esprit de corps all contributed to the formation of a cohesive cohort, providing support to students and aiding in their individual and collective successes.

The final survival aid according to the data was programmatic reassurance that they were not alone-reminders that others had experienced similar challenges during their medical education.

I think what's most helpful is frequent reassurance that what we're going through is normal. Checking in, making sure that things are going okay and reminding us that (what we are experiencing) is normal is a good thing. It helped me and it helped the group.

A favorite quote that we heard was "This too will pass." That was my absolutely favorite quote and I remember during times of being low, stressed or burned out, that was all I could think about.

Having the faculty tell us, “This is hard but you'll get through it. Look back (on how far you have come) rather than how far you have left to go" - that was so helpful. 
These comments by study participants highlight potential programmatic interventions that could smooth the process for future PA students-interventions that are discussed further in chapter 5 .

\section{Moving Out and Moving On}

As the focus group sessions came to a close, the discussion among the study participants took on the tenor of a goodbye as they looked toward their future career trajectories. The intense first year of their medical education had been completed and was now in the past. Remembrances of the didactic year were still fresh in their minds as they were only a few months away from the experience, but they recognized that their views of the experience might change over time as they moved on with their education, their careers and their lives.

PA school is a lot like the military or the Peace Corp. It's similar in that the days dragged but the weeks fly by. The further you get from the bad times the less bad you remember.

We will think back on that first year and those odd moments and fun times and ridiculous things that we did and then we'll forget the hard journey we all traveled together.

At this point I can't say I'll miss it, but ... give it a year and I will miss it. I know I will miss it because the same thing happened in the military when I went to Iraq. I went through deployment and it was really intense and I suffered through it like last year (in PA school)_-just in a different way. I ended up missing (the military) and I know I am going to miss everyone around this table. I'll think back and say "what a good time we had last year."

And finally,

It was the best, most horrible time I have ever had. It was really hard physically and intellectually and emotionally. The best, worst time. 


\section{Summary: Transition, Change and Transformation}

In analyzing the data from the content analysis of the focus group sessions, a number of themes regarding the lived experience of the didactic year of PA education emerged. The major theme identifies the cohort educational experience as a three-stage process with a beginning, a middle and an end-a model that aligns with a number of stages-of-change theories from other professions and disciplines. The subthemes that came to light from the content analysis highlight a pattern of emotional facets within the three-stage experience, beginning with excitement and exhilaration, followed by Imposter Syndrome, culture shock, stages of grieving, isolation and guilt, loss of competence and confidence, demoralization and the potential for burnout and other forms of psychological distress. Finally, excitement returns as confidence improves and competencies begin to develop. These emotional subthemes also align with other threestage models.

The transitions and change experiences of study participants during this threestage process lead to transformations in their personal as well as their role identitiestransformations that comprise significant outcomes of this intensive medical education process. Enculturation into a new professional identity is a key construct within the experience of the didactic year, and one that appears to follow the patterns illustrated in the Menninger (1975) Morale Curve, the U-Curve of Cross Cultural Adjustment (Gullahorn \& Gullahorn, 1963), Moir’s (1999) Phases of First-Year Teacher’s Attitudes, and other three-stage models of change described previously. Whereas once undifferentiated, by the completion of the didactic year of their PA education, 
participants are now beginning to be socialized into the role of a health care provider, and once in this role, an individual can never return to their previous status; thinking as a clinician is not something that can be unlearned. As Robertson (1997) said, "You cannot simply add the notion that the world is round to the notion that the world is flat. Some types of learning clearly have this transformative effect ...” (p. 109).

The process of joining a new profession such as medicine is similar to the assimilation process into a new culture, and a predominant feature of assimilation models is the patterned role that emotion plays as individuals move through the change process. Menninger (1975) believed that when life circumstances change, an individual's “sense of himself undergoes changes that produce the stresses reflected by the morale curve” (p. 110). The construct of change, then, is an important mediator for morale, and the morale curve may be the graphic representation of the human experience of transformative change. Morale, then, could be a barometer for the stressors inherent in the transition itself. One of the questions posed in chapter 2 was, "Are the observed shifts in student cohort morale a function of transformative change?” It is my assertion that the data from this research project confirms just such a pattern of morale and transformative change through the didactic year of PA education and matches other three-stage models as depicted in Figure 8. 


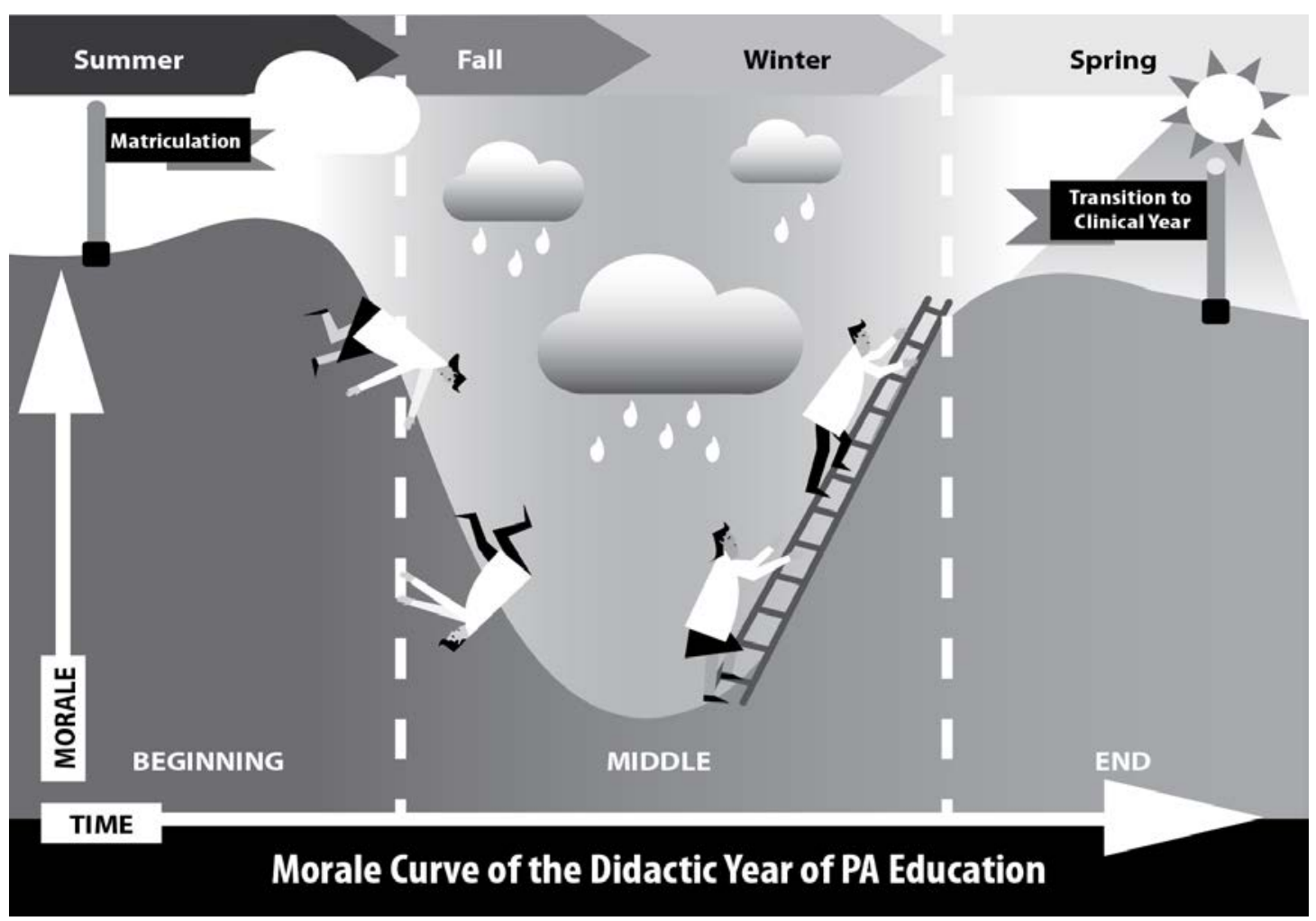

Figure 8. The morale curve of the didactic year of PA education.

There appears to be a great deal of consistency regarding the experience of change and transformation across several diverse disciplines of study. All of these models describe the same phenomena with overlapping concepts — change, transition, the emotional journey of transformation and personal growth, the development of competence and autonomy. Data from this study supports the existence of a stages-ofchange model in PA education; the content analysis clearly describes the student perspective corroborating the existence of a three-stage experience with emotional overtones as they move through their didactic medical education in the study program. Such a model has not been previously applied to medical education, and while this current study involves a single cohort of PA students at a single university, the results 
reinforce anecdotal observations by faculty in this as well as other PA programs. This research offers an initial investigation into the experience of PA education from the perspective of the student and provides a lens through which the process of medical education can be conceived in future research. Further inquiry into the experience of PA education is imperative to enhance the effectiveness of the educational process while also attending to the effects of that educational process on students.

Education contributes to the transformation of individuals, and regardless of the type of transitional event, learning occupies a prominent role in personal and professional development. As the process of learning can have a significant emotional component, the way in which the educational process is made available to students can have far reaching effects on the assimilation of knowledge as well as emotional well-being. In the next chapter, I discuss the implications of this research on the practice of medical education with the goal of ameliorating student challenges, improving student learning and promoting the development of safe and effective PAs. 


\section{CHAPTER 5}

\section{IMPLICATIONS FOR PRACTICE}

The professional journey to become a PA is a rigorous, time-consuming and transformative process that includes medical education, clinical skills training and role socialization. Prior to this dissertation, little was known about the effects of this educational process on PA students from the perspective of the student; there has been a dearth of information on the experience of thousands of students who undertake this educational role transformation each year. The aim of this dissertation was to explore the experience of PA education at an academic health center on the West Coast, to identify common themes ascribed to those experiences from the student perspective and to enhance understanding about this understudied group while simultaneously giving students a voice in the educational process in which they are engaged. The overarching research questions were:

- What is the student experience of the didactic phase of physician assistant education?

- In what ways does this intense educational experience affect students?

- How does this experience affect student mood and morale?

Expedited IRB approval was obtained for this qualitative naturalistic focus group research project conducted with a purposive sample of 10 volunteer study participants who had completed the didactic year of the program. Audio recordings of the focus group sessions were professionally transcribed resulting in formal de-identified transcripts; microanalysis identified general initial categories by which to conceptualize common 
outcomes and experiences reported by students. Through a process of manual open and axial coding, themes and subthemes describing the phenomenon of the student experience were identified by the researcher.

\section{Summary of Findings}

This research brings to light the lived experience of the didactic year of medical education from the perspective of the study participants. The overarching theme of the cohort educational experience that arose from the data was that of a three-stage process with a beginning, a middle and an end-an experience that aligns with a number of stages-of-change theories from other professions and disciplines. The subthemes that were revealed from the content analysis of the data highlighted a pattern of emotional facets within this three-stage experience, beginning with excitement and exhilaration, followed by the Imposter Syndrome, culture shock, stages of grieving, isolation and guilt, loss of competence and confidence, demoralization and the potential for burnout and other forms of psychological distress. Finally, excitement returns as confidence improves and competencies begin to develop. These emotional subthemes also align with other three-stage models and are likely representative of the process of human transformation.

The transitions and change experiences of study participants during this threestage process lead to transformations in personal identity as well as role identitytransformations that comprise significant outcomes of this intensive medical education process. Enculturation into a new professional identity is a key construct within the experience of the didactic year, and one that appears to follow the patterns illustrated in each of the three-stage models of change described previously. Whereas once undifferentiated, by the completion of the didactic year of their PA education, 
participants are now beginning to be socialized into the role of a health care provider. The shared expressions from study participants richly describe the impact this transformative experience has, both personally as well as professionally, and as such, provide reassurance about this study’s trustworthiness and rigor. Because this is an initial investigation into the student experience of the didactic year of PA education, further discussion of the steps taken to address trustworthiness and rigor are important to set the stage for future research.

\section{Trustworthiness and Rigor}

Morrow (2005) described “standards of trustworthiness” (p. 250) that are essential to all qualitative research paradigms, and emphasized the importance of building a qualitative research design with trustworthiness in mind. Lincoln and Guba (1985) specified three measures that uphold the concept of trustworthiness within such a qualitative research paradigm—credibility, dependability and transferability. Credibility in this study was achieved through the utilization of student participants intimately involved in the educational process under investigation; these individuals were the ideal source from which to address the research questions as they are lived participants of the experience. They alone can speak to its effects more eloquently that any other data source, and as such are the main source of the credibility of the study results. While this research focused on members of a single student cohort, their experiences mirror those of other cohorts observed anecdotally by faculty in the study institution as well as others, further suggesting the credibility of study outcomes. Another aspect of credibility was the care taken throughout the analysis of the data. The selection of the meaning units—or quotations - in identifying the major theme and subthemes was a key factor in making 
sense of the narrative data. I attended to credibility within the coding process by reading and rereading the transcripts of the focus group sessions in their entirety several times to get a sense of the "whole," then grouped quotations based on emerging ideas. Once the content was organized, I again reread the documents in order to confirm or rework categories and concepts to assure an appropriate, accurate and rich accounting of student experiences. Every effort was taken to honestly reflect study participant comments knowing that I was providing the opportunity to communicate the importance of their life-changing educational experience through this research project.

In consideration of the dependability of the data, it was essential to recognize the impact of timing on the collection of student retrospective recollections. The focus group sessions were scheduled to occur approximately six months after completion of the didactic year-a decision made with intentionality in order to allow study participants adequate time to develop a sense of perspective on their didactic experience as a whole. It was also essential to conduct the sessions before students had moved too far along their educational and professional trajectory in order to avoid the potential evolution or decay of remembrances over time. Collection of information too soon or too late after completion of an experience could result in an alteration of memory. The group nature of the interviews, however, assisted students in recalling the shared nature of the experience and served to triangulate the data among participants in the study.

This shared experience, in my estimation, contributed to the dependability of the study results within the context of this particular cohort group. By their narratives, this was the experience of individuals in this particular cohort as well as that of the cohort as a whole. Is this also the experience of other PA students enrolled in other programs? 
Lincoln and Guba's (1985) last measure of trustworthiness, transferability, describes the potential to apply these outcomes to other groups. The major findings of this study, as evidenced by the rich commentary of study participants, include a clear identification of a three-stage experience with specific emotional subthemes that occur within the framework of this human change model. Given that other PA programs also incorporate medical education, clinical skills training and role socialization in their education of PA students, the nature of this experience is likely to be similar among other physician assistant programs across the United States. Formal inquiry has not yet occurred, however, anecdotal observations among faculty at other programs suggest that this phenomenon is not unique to students at the study program. While one should not generalize results of a single study to the PA student population as a whole, one can advance knowledge about a single group’s experience with the goal of seeking confirmation with other groups of students. Context dependent knowledge is critical in understanding the experience of the student. Transferability, then, seems likely but as yet unproven.

In addressing the notion of rigor, Padgett $(1998,2004)$ outlined six strategies for enhancement of rigor within a qualitative study, including prolonged engagement, triangulation, peer debriefing and support, member checking, negative case analysis and auditing. In this research study I relied heavily on several of these strategies to address issues with rigor - the first was triangulation. The triangulation utilized in this study took place within the focus group sessions among the study group participants. Frey and Fontana (1991) described group interviews as particularly helpful in the process of “indefinite triangulation” (p. 177) in which ideas are bounced back-and-forth between 
individuals and the modified by the group, leading to a greater elaboration of statements beyond that provided by a single individual. The "polyphonic” (Frey \& Fontana, 1991, p. 178) data collected represents a broader spectrum of participant opinions while diffusing the influence of the interviewer within the group setting. The study participants set the tone for rigor; my goal as a researcher was to accurately capture the essence of their experience. The second strategy I utilized was member checking. Upon receipt of the professional transcriptions and after the subsequent content analysis, I engaged in individual member checking among the focus group participants in order to determine whether my interpretations captured the meaningfulness of the conversations. I also called upon Padgett’s (1998) strategy of prolonged engagement. As a participant observer in the process of physician assistant education for more than 25 years, first as a student and subsequently as an educator, I have had the good fortune of sharing educational experiences with an annual series of student cohorts. It is from this prolonged engagement that the concept of this study arose; having lived the experience of PA education myself, it was important to me personally that the student experience be brought to light. Finally, I engaged in peer debriefing with faculty and staff at the study program in order to check my perceptions of themes emerging from the data. Overall, trustworthiness and rigor were considered throughout the design, implementation and analysis of this research project.

\section{Strengths of This Research}

Prior to this study, the student experience of the didactic year of PA education had not been examined in detail and the voice of the student was unheard. This study represents an initial inquiry into the experience of an intensive medical education process 
designed to prepare safe and effective health care providers, and as such, adds to our understanding of the magnitude of the experience on students engaged in that medical education process. Through the collection and analysis of the powerful data derived from study participants during the focus group sessions, a three-stage model that mirrors other human stages of change models was identified. This outcome has the potential to inform medical educators and academic institutions about the student experience, and suggests future curricular interventions to enhance and improve student learning while being mindful of the impact of the experience on the emotional well-being of student participants. Research opportunities related to the student experience of an educational process that involves transformative change, professional identity formation, and psychological distress are more clearly identified.

\section{Limitations of This Research}

Limitations of this study include the small number of participants representing a single institution, constraining the degree to which transferability can be considered. While these results ring true for this particular group, applying the results to PA students in other programs is premature and awaits further study. The midpoint of the year represents the "bottoming out" of student morale and was identified as the most difficult period within the didactic phase among the study participants. In the study program, this timeframe correlates with the late fall through the winter months. Would this low point occur at the same time in an educational setting that had milder winters? Or one in which the middle of the didactic year occurred during a different season? Additional research could answer these questions and would add to the potential of transferability. 
Volunteer bias should be considered a potential given the self-selection process, and if present could have the capacity to impact the results. All study participants were volunteers and may have chosen to participate due to a personal agenda-either positive or negative. Inclusion of additional students from that same class cohort could have allowed for more varied voices, but would have also added to the complexity due to the volume of the narrative data. Data collection from additional student groups in the future would mitigate the impact of potential volunteer bias. The timing of the focus groups may have been a limitation in that data collection was retrospective and accumulated during a narrow window of time. While this study was intentionally designed to capture student impressions of the whole of the didactic year, the results might have been different if study participants had been assessed at multiple points throughout their educational experience. In the future it would be worth considering a longitudinal mixedmethods inquiry throughout the didactic year to more specifically identify student impressions at given points in time. Another limitation related to the study design might exist due to cohort effect. Cozby (2009) described the cohort effect as a phenomenon depicting the impact that experiences and social influences have on a group of people within a particular age range. Major social, political and world events have the potential to influence the results of a cohort study, particularly if cross sectional. In this case, however, the study cohort included subjects from a variety of ages who were all exposed to similar educational experiences, thus lessening the likelihood of a cohort effect. Another potential limitation could be the preconceived notions of the researcher. I acknowledge the biases I hold as a result of being imbedded into the process of PA education for many years, however, and attempted to clarify that bias while also avoiding 
imposition of my experiences over those of the study participants. And finally, while some might have concern about the potential for bias confirmation with a naturalistic focus group study design, the potential in this instance should be no different than in other forms of qualitative research.

\section{Programmatic Interventions}

As a result of this study, there are a number of potential implications for practice due to the many educational and emotional challenges faced by students. The student experience of the didactic year mirrors three-stage models of human change with a beginning, a middle and an end; the Menninger (1988) Morale Curve provides a description of the emotional nature of professional identity formation inherent within that human change process. If this pattern holds true for PA student cohorts in general, it has the potential to guide programs in the development of institutional processes that can ameliorate the challenges of this intensive medical education program while simultaneously maintaining the high standards necessary for the safe and effective care of patients.

As reported in the discussion of the data, study participants highlighted three thematic areas that they believe contributed to their success during the didactic phase of the program-the important role of the cohort, the lack of competition, and programmatic reassurance. Each of these is worthy of exploration in order to identify potential programmatic interventions in support of student success.

\section{The Role of the Cohort}

There is a growing body of scholarship regarding the importance of the cohort model, particularly as it relates to the development of student learning communities 
(Hesse \& Mason, 2005; Mello, 2003). “The school is a cohort creator” (Ryder, 1965, p. 854), and the creation of cohort communities founded upon collaborative learning activities, as in PA education, provides a shared culture that evolves from time spent together on a common goal. This culture "socializes students to the professional practices of teamwork and collaboration” (Sathe, 2009, p. 6). Mandzuk, Hasinoff, and Seifert (2003) described the cohort as a place where students are prepared for future professional roles in which "their social networks will include dense, overlapping professional and social relationships” (p. 172), a circumstance that is true for health care providers working in complex medical communities in the inpatient and outpatient settings. Students believe the likelihood of successful completion of their program is greater due to the support offered by their cohort (Hesse \& Mason, 2005; Mello, 2003); a notion reiterated by comments from study participants in this research. Interpersonal skills as well as group work skills are imperatives of a professional role in medicine, and the cohort model in medical education can provide the sandbox in which novices can set aside their individualistic orientation in favor of skill enhancement within the context of a group. A shared experience, a common goal, a noncompetitive environment and esprit de corps all contribute to the formation of a cohesive cohort providing support to students and aiding in their individual and collective successes. Team spirit has been defined as "the extent to which individuals feel a sense of group togetherness and participate in the team dynamics” (Silva et al., 2013, p. 2). The components necessary for the maintenance of positive morale, or team spirit, have been postulated to include a functional support system, a grounded perspective where the individual learns from the past but focuses on the future, a realistic outlook (neither optimistic nor pessimistic) and the ability to assume 
personal responsibility for one’s actions (Richards, 1999). In order to encourage and maintain positive morale among a cohort of students, programs should begin by providing an informed consent process for incoming students-a type of anticipatory guidance that can prepare students for the ups and downs of the educational process ahead. "Newcomers with unmet role expectations are predicted to suffer the dysfunctional effects of reality shock” (Major, Kozlowski, Chao, \& Gardner, 1995, p. 418). A number of studies highlight the importance of assimilation to the socialization process, and not only recommend an initial orientation, but an ongoing one as well (Ahronson \& Cameron, 2007; Coombs et al., 1990; Gardner \& Barnes, 2007; Louis, Posner, \& Powell, 1983; Major et al., 1995; Schor, Guillet, \& McAnarney, 2011). As the Latin proverb states, Praemonitus, praemunitas_forewarned, forearmed.

\section{Minimizing Competition}

In order to develop a functional and cohesive cohort, competition is an issue that must be addressed. PA education is unique in that student achievement is measured by successful completion of programmatic requirements followed by successful passage of the national certification examination. As both of these are criterion-referenced goals, competition is not a requirement. Medical students, on the other hand, maintain their competitive nature as they must compete for residency opportunities upon graduation from medical school ... and the competition is fierce (Hester, 2001; Jolly, Erikson, \& Garrison, 2013). In order to downplay competition within a PA student cohort, programmatic structures that value competition over collaboration should be addressed, with the goal of encouraging students to learn by, with and from each other, ensuring the success of the entire cohort. Helping students recognize that competition does not help 
them succeed in this new medical education endeavor allows a collective relationship to develop within the cohort-a collaborative process that is inherently part of the professional PA role as a health care provider.

\section{Programmatic Reassurance}

Study participants specifically identified episodic reassurance from the program as a key measure in their achievement of success; they likewise appreciated reminders that the emotional nature of their experience was not unusual. Informing students that others have experienced similar challenges during their medical education and succeeded despite self-doubt and feelings of imposter syndrome can provide encouragement and may reduce psychological distress. In addition to providing informed consent about the educational process about to be undertaken, students should also be made aware of the process of professional identity formation and its effects. Holden et al. (2015) described professional identity formation as "the transformative journey through which one integrates the knowledge, skills, values and behaviors of a competent, humanistic physician with one's own unique identify and core values” (p. 2)-or, the development of new ways of being. While students arrive at their new educational experiences anticipating a change in their professional role, they do not expect to undergo a personal change process. Many are not aware of the developmental nature of professional identity formation and may not be prepared for the challenging emotional nature of the liminal state as they adopt the mantle of a new profession. Students travel a path from high functioning and capable adult to a novice in a new role, with each step requiring mastery before moving on to the next step. A didactic year student may struggle with the sense of incompetence during the classroom year but then will adapt and master the process, 
moving on to the clinical student role, once again placing them into the role of a novice (Jarvis-Selinger et al., 2012; Sharpless et al., 2015; Wald, 2015). Describing the identity formation process to students may provide a measure of comfort about their experience (Sharpless et al., 2015; Wald, 2015).

In addition to explicit discussions about the student experience of the educational process, programs need to address the potential that psychological distress may develop. As it is well accepted that many health professions students experience stress as part of a medical education process (Finkelstein et al., 2007; Henning et al., 1998; Schmitter et al., 2008), and stress has been recognized to have a negative effect on academic performance (Spiegel et al., 1986), health and psychological well-being (Helmers et al., 1997), it behooves programs to be alert to psychological distress among students. Attending to the group dynamics of the cohort may positively impact psychological well-being; a strong measure of group cohesion is associated with lower levels of psychological distress (Bliese \& Halverson, 1996). Group social cohesion enhances the sense of belongingness, which may bolster adjustment to this new educational experience.

In order to address challenges to student well-being, some medical education programs have implemented wellness curricula designed to assist students in their development of healthy coping skills (Cox et al., 2001; Finkelstein et al., 2007; Laird, Hawkins, Hertweck, \& Swanson-Biearman, 2006; Rosenzweig, Reibel, Greeson, Brainard, \& Hojat, 2003). Anxiety and stress management, mental health and medical health care, exercise and fitness strategies all have the potential to enhance the health and well-being of students and may also reduce the incidence of burnout (Awa, Plaumann, \& Walter, 2009; Fredrickson, 2001; Maslach \& Goldberg, 1998; Perry \& Purka, 2008; 
Styles, 1993; Ziegler, Kanas, Strull, \& Bennet, 1984). Primary prevention of psychological distress is a key component of healthy professional development, and preventive efforts designed to assist students in the avoidance of psychological pitfalls are preferable to rehabilitative efforts once psychological distress has occurred. Setting realistic expectations upon matriculation, providing positive mentoring relationships and encouraging a culture of collaboration, support and resilience are all interventions that may even out the ups-and-downs of the medical education experience.

In addition to interventions at the student affairs level, there are implications for practice at the program administration and curricular levels as well. A review of policies and procedures can ascertain whether current administrative practices have the potential to exacerbate student challenges. An alteration of current policies or development of new policies could ameliorate student challenges without compromising the goal of providing a rigorous medical education designed to develop safe and effective health care providers. Several administrative areas deserve investigation, including:

- A review of accreditation requirements. Although general course content and outcomes are mandated by national accreditation standards (Accreditation Review Commission, ARC-PA), the number of contact hours and the configuration of those educational processes are not. Opportunities to ease course overload and mitigate stress and fatigue may be identifiable on review of program policies and accreditation standards.

- A review of curricular depth, pacing and sequencing across each course, each term and over the entire program. Removal of unintentional repetitive information should be a priority. As new material is added, a concerted effort must be undertaken to remove unnecessary material in order to prevent student overload, keeping in mind the issue of student workload. A policy regarding protected time should be drafted, agreed upon and then held sacred in order to decrease excessive "seat time," allowing assimilation of class material through reading, research, introspection and reflection. 
- An honest appraisal of internal departmental politics. The varied goals of the many stakeholders involved in medical education may impact and overload the curriculum without providing additive value to student learning. Conflicts among stakeholders should be identified and resolved with the student's best interests in mind.

- A review of student policies and procedures for the program. Policies that deal with attendance and deceleration, in particular, can create punitive outcomes or flexible solutions depending on the verbiage and application.

- Access to student health services. While enrollees typically have access to campus-based health services, dense course schedules may make it difficult for students to avail themselves of those services. Policies addressing student access to psychological services, substance abuse treatment and medical leaves of absence need to be in place to provide a safe haven for students needing access to such resources, and protected time for students to utilize medical and/or psychological services if needed is imperative. Faculty and staff should receive training designed to identify maladaptive responses to the educational process in order to facilitate appropriate supportive interventions.

- Access to tutoring and educational support services. At the graduate/doctoral level, there is often an assumption that, once admitted, students will be capable of completing the work without intervention given their prior record of academic success. Given the pace and density of material in a graduate medical education program, students may experience academic challenges not previously identified in the undergraduate setting. Availability of academic services is necessary to support student success.

A number of implications for practice emerge from this research. Opportunities

for the development and implementation of well-designed interventions from the curricular, student services and program administration perspectives have the potential to positively impact the student experience during the medical education process. As more is learned about the effects of PA education on students, opportunities to view PA education from a much broader perspective than just the delivery of medical knowledge will likewise be enhanced. 


\section{Conclusion}

Physician assistant students appear to undergo profound change as they transition into the health care provider role through their transformational medical education journey; their perspectives are different by the time they reach the end of the program having developed sophisticated new ways of thinking and solving problems. As a member of a faculty charged with guiding the medical education process, I have observed the transformation of students through their educational experience and recognize the emotional nature of this transformation as expressed through the barometer of mood and morale. This dissertation served as an initial investigation into a set of ideas drawn from the existing literature grounded in social constructivism, transformational learning, role socialization, identity and professional development, and the human change process. This study highlights the similarities in models across a number of disparate disciplines, and adds the medical education of physician assistants to those models as another example of the transformational human change process. While the findings of this study are constrained by the conditions inherent in an inquiry involving a single cohort of students at a single university, they provide a previously undocumented description of the experience from the perspective of the student. This research emphasizes the need for additional investigations in order to enhance and expand our understanding of the student experience and to confirm the presence of similar patterns of mood and morale among students at PA programs across the United States.

The process of medical education is rigorous and challenging yet should not be inhumane. While PA Program faculty and staff are under pressure to meet the expectations of external accrediting and licensing bodies, compelling the delivery of this 
fire hydrant experience, we must seek continuously seek opportunities to balance the process in order to improve the educational experience for students. Attending to patterns of mood and morale as a measure of the human change process may allow anticipation of student challenges before they arise, and the successful completion of program requirements by our student cohorts can be enhanced by mitigation of the psychological impact that programs have on students. Wherever possible, palliation of stress, anxiety, demoralization and other measures of psychological distress can be programmatic goals even while maintaining academic standards necessary for the development of safe, effective and psychologically sound physician assistants. Our students’ future patients deserve nothing less in their health care providers. 


\section{REFERENCES}

Accreditation Review Commission on Education for the Physician Assistant. (2010). Accreditation standards for physician assistant education ( $4^{\text {th }}$ ed.). Retrieved January 20, 2011, from http://www.arc-pa.org/Standards/standards.html

Adler, P. S. (1975). The transitional experience: An alternative view of culture shock. Journal of Humanistic Psychology, 15(4), 13-22.

Ahronson, A., \& Cameron, J. E. (2007). The nature and consequences of group cohesion in a military sample. Military Psychology, 19(1), 9-25.

American Academy of Physician Assistants. (2010). How the new health care reform law impacts PAs. Retrieved February 9, 2011, from http://www.aapa.org/ advocacyand-practice-resources

American Academy of Physician Assistants. (2011). Our history. Retrieved January 28, 2011, from http://www.aapa.org/about-pas/our-history

American Academy of Physician Assistants. (2014). PAs practice medicine. Retrieved January 14, 2016, from AAPA_FactSheets_PAsPractice_10.14FINAL.pdf

Atchley, R. C. (1976). The sociology of retirement. Cambridge, MA: John Wiley \& Sons.

Awa, W. L., Plaumann, M., \& Walter, U. (2009). Burnout prevention: A review of intervention programs. Patient Education and Counseling, 78, 184-190.

Ballweg, R., Sullivan, E. M., Brown, D., \& Vetrosky, D. (2008). Physician assistant: A guide to clinical practice. Philadelphia, PA: Saunders Elsevier.

Bandura, A. (1977). Social learning theory. Englewood Cliffs, NJ: Prentice-Hall.

Bandura, A. (1982). Self-efficacy mechanisms in human agency. American Psychologist, 37(2), 122-147.

Bellini, L. M., Baime, M., \& Shea, J. A. (2002). Variation of mood and empathy during internship. Journal of the American Medical Association, 287, 3143-3146.

Benner, P. (1982). From novice to expert. American Journal of Nursing, 82, 402-407.

Biddle, B. J. (1979). Role theory: Expectations, identities and behaviors. New York, NY: Academic Press. 
Black, J. S., \& Mendenhall, M. (1991). The U-curve adjustment hypothesis revisited: A review and theoretical framework. Journal of International Business Studies, 22, 225-247.

Bliese, P. D., \& Halverson, R. R. (1996). Individual and nomothetic models of job stress: An examination of work hours, cohesion and well-being. Journal of Applied Social Psychology, 26, 1171-1189.

Brazeau, C. M., Schroeder, R., Rovi, S., \& Boyd, L. (2010). Relationships between medical student burnout, empathy and professionalism climate. Academic Medicine, 85(10 Suppl), S33-36.

Bridges, W. (1985, September). How to manage organizational transition. Training, 30-34.

Bridges, W. (1991). Managing transition: Making the most of change. Reading, MA: Addison-Wesley

Bridges, W. (2003). Managing transitions: Making the most of change. Philadelphia, PA: DeCapo.

Brown, L., \& Brown, J. (2009). Out of chaos, into a new identity: The transformative power of the international sojourn. Existential Analysis, 20(2), 341-361.

Brown, M. H. (2004). The increased awareness of physician assistant student mental health. Perspective on Physician Assistant Education, 15(2), 116-120.

Burch, N. (1970). Four stages of learning. Retrieved November 28, 2015, from http://www.gordontraining. com/free-workplace-articles/learning-a-new-skill-iseasier-said-than-done

Bureau of Labor Statistics, U.S. Department of Labor. (2015). Career outlook handbook (2015 ed., Physician Assistants). Retrieved January 15, 2016, from http://www.bls.gov/careeroutlook/2015/article/projections-occupation.htm.

Burton, J. (2006). Transformative learning: the hidden curriculum of adult life. Work Based Learning in Primary Care, 4, 1-5.

Cardall, W. R., Rowan, R. C., \& Bay, C. (2008). Dental education from the student's perspective: Curriculum and climate. Journal of Dental Education, 72, 600-609.

Casey, M. A., \& Drueger, R. A. (1988). Focus group interviewing. In H. MacFie \& D. Thomson (Eds.), Measurement of food preferences (pp. 77-96). Hong Kong: Springer Science + Business Media. 
Cawley, J. F. (2007). Physician assistant education: An abbreviated history. Journal of Physician Assistant Education, 18(3), 6-16.

Cawley, J. F., Cawthon, E., \& Hooker, R. S. (2012). Origins of the physician assistant movement in the United States. Journal of the American Academy of Physician Assistants, 25(12), 36-42.

Cecil, J., McHale, C., Hart, J., \& Laidlaw, A. (2014). Behaviour and burnout in medical students. Medical Education Online, 19. Retrieved from http://dx.doi.org/ 10.3402/meo.v19.25209

Chickering, A. W. (1969). Education and identity. San Francisco, CA: Jossey-Bass.

Childers, W. A., May, R. K., \& Ball, N. (2012). An assessment of psychological stress and symptomatology for didactic phase physician assistant students. Journal of Physician Assistant Education, 23(4), 35-39.

Choy, S. (2009). Transformative learning in the workplace. Journal of Transformative Education, 7(65), 65-83.

Chrisman, S. M., Pieper, W. A., Clance, P. R., Holland, C. L., \& Glickauf-Hughes, C. (1995). Validation of the Clance imposter phenomenon scale. Journal of Personality Assessment, 65(3), 456-467.

Clance, P. R., \& Imes S. (1978). The imposter phenomenon in high achieving women: Dynamics and therapeutic intervention. Psychotherapy Theory, Research and Practice, 15(3), 1-8.

Clark, D., \& Kissane, D. (2002). Demoralization: Its phenomenology and importance. Australian and New Zealand Journal of Psychiatry, 36, 733-742.

Clark, D. C., \& Zeldow, P. B. (1988). Vicissitudes of depressed mood during four years of medical school. Journal of the American Medical Association, 260(17), 25212528.

Cleland, J., \& Johnston, P. (2012). Enculturation to medicine: Power for teachers or empowering learners? Medical Education, 46, 835-837.

Coombs, R. H., Perrell, K., \& Ruckh, J. M. (1990). Primary prevention of emotional impairment among medical trainees. Academic Medicine, 65,576-581.

Cox, C. C., Hooper, J., Cambre, K. M., Worlf, T. M., Jones, A., \& Danielsen, R. (2001). Scope of health promotion programs for physician assistant students. Perspective on Physician Assistant Education, 12(1), 13-16. 
Cozby, P. C. (2009). Methods of behavioral research (10 ${ }^{\text {th }}$ ed.). New York, NY: McGraw-Hill.

Cozzarelli, C., \& Major, B. (1990). Exploring the validity of the imposter phenomenon. Journal of Social and Clinical Psychology, 9, 401-417.

Cranton, P. (1998). Transformation, not training. Adult Learning, 9(3), 14-16.

Dahlin, M., Joneborg, N., \& Runeson, B. (2005). Stress and depression among medical students: A cross sectional study. Medical Education, 39, 594-604.

Daley, B. J. (1999). Novice to expert: An exploration of how professionals learn. Adult Education Quarterly, 49(4), 133-147.

de Figueiredo, J. (1983). Some issues in research on the epidemiology of demoralization. Comprehensive Psychiatry, 24(2), 154-157.

de Figueiredo, J. (1993). Depression and demoralization: Phenomenologic differences and research perspectives. Comprehensive Psychiatry, 34(5), 308-311.

de Figueiredo, J., M., \& Frank, J., D. (1982). Subjective incompetence, the clinical hallmark of demoralization. Comprehensive Psychiatry, 23(4), 353-362.

Deutsch, M. (1954). Field theory in social psychology . In G. Lindzey \& E. Aronson (Ed.), The handbook of social psychology (Vol. 1; 2nd ed.; pp. 412-487). Reading, MA: Addison-Wesley.

Dickstein, L. J., Stephenson, J. J., \& Hinz, L. D. (1990). Psychiatric impairment in medical students. Academic Medicine, 65(9), 588-590.

Dictionary.com. (n.d.a). Dictionary. Confidence. Retrieved January 18, 2016, from http://dictionary.reference.com/browse/confidence

Dictionary.com. (n.d.b.). Dictionary. Demoralization. Retrieved December 16, 2015, from Dictionary.reference.com/browse/demoralization.

Downe-Wamboldt, B. (1992). Content analysis: Method, applications and issues. Health Care for Women International, 13, 313-321.

Dreyfus, S. E. (2004). The five-stage model of adult skill acquisition. Bulletin of Science, Technology and Society, 24(3), 177-181.

Dreyfus, S. E., \& Dreyfus, H. L. (1980). A five-stage model of the mental activities involved in directed skill acquisition. Operations Research Center, University of California, Berkeley. 
Dyrbye, L. N., Massie, F. S., Eacker, A., Harper, W., Power, D., \& Shanafelt, T. D. (2010). Relationship between burnout and professional conduct and attitudes among US medical students. Journal of the American Medical Association, 304, 1173-1180.

Dyrbye, L. N., Thomas, M. R., Huntington, J. L., Lawson, K. L., Novotny, M. S., Sloan, J. A., \& Shanafelt, T. D. (2006). Personal life events and medical student burnout: A multicenter study. Academic Medicine, 81, 374-384.

Dyrbye, L. N., Thomas, M. R., Massie, F. S., Power, D. V., Eacker, A., Harper, W., \& Shanafelt, T. (2008). Burnout and suicidal ideation among U.S. medical students. Annals of Internal Medicine, 149(5), 334-341.

Dyrbye, L. N., Thomas, M. R., \& Shanafelt, T. D. (2006). Systematic review of depression, anxiety and other indicators of psychological distress among U.S. and Canadian medical students. Academic Medicine, 81(4), 354-373.

Elrod, P. D., \& Tippett, D. D. (2002). The "death valley" of change. Journal of Organizational Change Management, 15(3), 273-291.

Epstein, R. M., \& Hundert, E. M. (2002). Defining and assessing professional competence. Journal of the American Medical Association, 287(2), 226-235.

Erikson, E. (1956). The problem of ego identity. Journal of the American Psychoanalytic Association, 4, 56-121.

Erikson, E. (1980). Identity and the life cycle. New York, NY: W. W. Norton \& Company.

Estes, R. J., \& Wilensky, H. L. (1978). Life cycle squeeze and the morale curve. Social Problems, 25(3), 277-292.

Finkelstein, C., Brownstein, A., Scott C., \& Lan, Y. (2007). Anxiety and stress reduction in medical education: An intervention. Medical Education 41, 258-264.

Frank, J. D. (1974). Psychotherapy: The restoration of morale. American Journal of Psychiatry, 131, 271-274.

Fredrickson, B. L. (2001). The role of positive emotions in positive psychology. American Psychologist, 50(3), 218-226.

Freudenberger, H. J. (1974). Staff burn-out. Journal of Social Sciences, 30, 159-165.

Frey, J. H., \& Fontana, A. (1991). The group interview in social research. Social Science Journal, 28, 175-187. 
Gardner, S. K., \& Barnes, B. J. (2007). Graduate student involvement: Socialization for the professional role. Journal of College Student Development, 48(4), 369-387.

Glicken, A. D., \& Gray, J. (1993). Adaptation of PA students to medical training. Journal of the American Academy of Physician Assistants, 6(6), 442-448.

Glicken, A. D., \& Miller, A. A. (2013). Physician assistants: From pipeline to practice. Academic Medicine, 88(12), 1883-1889.

Graneheim, U. H., \& Lundman, L. (2004). Qualitative content analysis in nursing research: Concepts, procedures and measures to achieve trustworthiness. Nurse Education Today, 24(2), 105-112.

Guba, E. G., \& Lincoln, Y. S. (2005). Paradigmatic controversies, contradictions, and emerging confluences. In N. K. Denzin \& Y. S. Lincoln (Eds.), The Sage handbook of qualitative research ( $3^{\text {rd }}$ ed.; pp. 191-216). Thousand Oaks, CA: Sage.

Gullahorn, J. T., \& Gullahorn, J. E. (1963). An extension of the U-curve hypothesis. Journal of Social Issues, 19(3), 33-47.

Hatch, J. A. (2002). Doing qualitative research in education settings. Albany, NY: State University of New York Press.

Helmers, K. F., Danoff, D., Steinert, Y., Leyton, M., \& Young, S. N. (1997). Stress and depressed mood in medical students, law students and graduate students at McGill University. Academic Medicine, 72(8), 708-714.

Henning, K., Ey, S., \& Shaw, D. (1998). Perfectionism, the imposter phenomenon and psychological adjustment in medical, dental, nursing and pharmacy students. Medical Education, 32, 456-464.

Hesse, M., \& Mason, M. (2005). The case for learning communities. Community College Journal, 76(1), 30-35.

Hester, D. M. (2001). Rethinking the residency matching process and questioning the value of competition in medicine. Academic Medicine, 76(4), 345-347.

Hewitt, P. L., \& Flett, G. L. (1991). Perfectionism in the self and social contexts: Conceptualization, assessment and association with psychopathology. Journal of Personality and Social Psychology, 60(3), 456-470.

Hiatt, J. (n.d.). The definition and history of change management. Retrieved January 14, 2016, from http://www.change-management.com/tutorial-people-side-mod1.htm 
Hojat, M., Mangione, S., \& Nasca, T. (2004). An empirical study of decline in empathy in medical students. Medical Education, 38, 934-941.

Holden, M. D., Buck, E., Luk, J., Ambriz, F., Boisaubin, E. V., Clark, M. A., . . . Dalyrmple, J. L. (2015). Professional identity formation: Creating a longitudinal framework through TIME (transformation in medical education). Academic Medicine, 90(6), 761-767.

Holland, K., Middleton, L., \& Uys, L. (2012). Professional competence: A concept analysis. Scandinavian Journal of Occupational Therapy, 19(2), 214-224.

Hooker, R. S., \& Cawley, J. F. (2003). Physician assistants in American medicine (2 $^{\text {nd }}$ ed). Philadelphia, PA: Churchill Livingstone.

Hsieh, H.-F., \& Shannon, S. E. (2005). Three approaches to qualitative content analysis. Qualitative Health Research, 15(9), 1277-1288.

IsHak, W., Nikravesh, R., Lederer, S., Perry, R., Ogunyemi, D., \& Bernstein, C. (2013). Burnout in medical students: a systematic review. Clinical Teacher, 10, 242-245.

Jarvis-Selinger, S., Pratt, D., \& Regehr, G. (2012). Competency is not enough: Integrating identity formation into the medical education discourse. Academic Medicine, 87(9), 1-6.

Jolly, P., Erikson, C, \& Garrison, G. (2013). U.S. graduate medical education and physician specialty choice. Academic Medicine, 88(4), 468-474.

Kingry, M. J., Tiedje, L. B., \& Friedman, L. L. (1990). Focus groups: A research technique for nursing. Nursing Research, 39(2), 124-125.

Krasner, M. S., Epstein, R. M., Beckman, H., Suchman, A. L., Chapman, B., Mooney, C. J., \& Quill, T. E. (2009). Association of an educational program in mindful communication with burnout, empathy, and attitudes among primary care physicians. Journal of the American Medical Association, 302(12), 1284-1293.

Kreuger, R. A. (1988). Focus groups: A practical guide for applied research. Newbury Park, CA: Sage.

Krippendorff, K. (1980). Content analysis: An introduction to its methodology (The Sage Commtext Series). London, UK: Sage

Kubler-Ross, E. (1969). On death and dying. New York, NY: Macmillan.

Kuhn, L., Kranz, P. L., Koo, F., Cossio, G., \& Lund, N. L. (2005). Assessment of stress in physician assistant students. Journal of Instructional Psychology, 32, 167-177. 
Laird, J., Hawkins, S., Hertweck, M., \& Swanson-Biearman, B. (2006). Wellness in a physician assistant curriculum: an experiential model. Journal of Physician Assistant Education, 17(4), 5-11.

Lave, J. (1993). The practice of learning. In S. Chaiklin \& J. Lave (Eds.), Understanding practice: Perspectives on activity and context, 2000-2008 (pp. 3-34). Cambridge, UK: Cambridge University Press.

Lewin, K. (1947). Frontiers in group dynamics. Human Relations, 1, 5-41.

Lincoln, Y. S., \& Guba, E. G. (1985). Naturalistic inquiry. Newbury Park, CA: Sage.

Lindstrom, U. H., Hamberg, K., \& Johansson, E. E. (2011). Medical students’ experiences of shame in professional enculturation. Medical Education, 45, 1016-1024.

Louis, M. R., Posner, B. Z., \& Powell, G. N. (1983). The availability and helpfulness of socialization practices. Personnel Psychology, 36, 857-866.

Ludmerer, K. (1999). Time to heal: American medical education from the turn of the century to the era of managed care. New York, NY: Oxford University Press.

Lysgaard, S. (1955). Adjustment in a foreign society: Norwegian Fulbright grantees visiting the United States. International Social Science Bulletin, 7, 45-51.

Major, D. A., Kozlowski, S. W., Chao, G. T., \& Gardner, P. D. (1995). A longitudinal investigation of newcomer expectations, early socialization outcomes, and the moderating effects of role development factors. Journal of Applied Psychology, 80(3), 418-431.

Mandel, E. D., \& Schweinle, W. E. (2012). A study of empathy decline in physician assistant students at completion of first didactic year. Journal of Physician Assistant Education, 23(4), 16-24.

Mandzuk, D., Hasinoff, S., \& Seifert, K. (2003). Inside a student cohort: Teacher education from a social capital perspective. Canadian Journal of Education, 28(1/2), 164-184.

Marks, M. (2007). A framework for facilitating adaptation to organizational transition. Journal of Organizational Change Management, 20(5), 721-739.

Maslach, C. (1982). Burnout: The cost of caring. Englewood Cliffs, NJ: Prentice-Hall.

Maslach, C., \& Goldberg J. (1998). Prevention of burnout: New perspectives. Applied and Preventive Psychology 7(63), 63-74. 
Maslach, C., Schaufeli, W. B., \& Leiter, M. P. (2001). Job burnout. Annual Review of Psychology, 52, 397-422.

Mattie, C., Gietzen, J., Davis, S., \& Prata, J. (2008). The imposter phenomenon: Selfassessment and competency to perform as a physician assistant in the United States. Journal of Physician Assistant Education, 19(1), 5-12.

McGrath, J. E. (1976). Stress and behaviors in organizations, In M. Dunnett (Ed.), Handbook of industrial organizational psychology (pp. 1357-1395). Chicago, IL: Rand McNally.

Mead, M. (1937). Cooperation and competition among primitive peoples. New York, NY: McGraw-Hill.

Medical Group Management Association. (1996). Physician compensation and production survey: 1996 report based on 1995 data. Denver, CO: Author.

Mello, R. A. (2003). The integrated cohort program: An evaluation of a preprofessional course of study. The Educational Forum, 67(4), 354-363.

Menninger, W. W. (1975). The meaning of morale: A Peace Corp model. In D. P. Moynihan (Ed.), Business and society in change (pp. 97-110). New York, NY: American Telephone \& Telegraph Company.

Menninger, W. W. (1988). Adaptation and morale: Predictable responses to life changes. Bulletin of the Menninger Clinic, 52(3), 198-210.

Merriam-Webster. (n.d.a.).Merriam-Webster's online dictionary $\left(12^{\text {th }}\right.$ ed). Cohort. Retrieved December 28, 2015, from http://www.merriamwebster.com/dictionary/cohort

Merriam-Webster. (n.d.b.). Merriam-Webster's online dictionary $\left(12^{\text {th }} \mathrm{ed}\right)$. Morale. Retrieved September 16, 2011, from http://www.m-w.com/dictionary/morale

Mezirow, J. (1997). Transformative learning: Theory to practice. New Directions for Adult and Continuing Education, 74, 5-12.

Mezirow, J. (2000). Learning as transformation. San Francisco, CA: Jossey-Bass.

Michalec, B. (2010). An assessment of medical school stressors on preclinical students' levels of clinical empathy. Current Psychology, 29(3), 210-221.

Moir, E. (1999). The stages of a teacher's first year. In M. Scherer (Ed.), A better beginning: Supporting and mentoring new teachers (pp. 19-26). Alexandria, VA: Association for Supervision and Curriculum Development. 
Morgan, D. L. (1997). Focus groups as qualitative research $\left(2^{\text {nd }}\right.$ ed.). Thousand Oaks, CA: Sage.

Morgan, D. L., \& Spanish, M. T. (1984). Focus groups: A new tool for qualitative research. Qualitative Sociology, 7, 253-270.

Morrow, S. L. (2005). Quality and trustworthiness in qualitative research in counseling psychology. Journal of Counseling Psychology, 52(2), 250-260.

Myers, J. K., Lindenthal, J. J., \& Pepper, M. P. (1975). Life events, social integration and psychiatric symptomatology. Journal of Health and Social Behavior, 16(4), 421-427.

National Commission on the Certification of Physician Assistants. (2014). Competencies for the physician assistant profession. Retrieved January 14, 2016, from http://www.nccpa.net/pdfs/Definition\%20of\%20PA\%20Competencies\%203.5\%2 0for\%20Publication.pdf

Nurcombe, B., \& Ebert, M. H. (2008). The psychiatric interview. In M. H. Ebert, P. T. Loosen, B. Nurcombe, \& J. T. Leckman (Eds.), Current diagnosis and treatment: Psychiatry (2 ${ }^{\text {nd }}$ ed.).Retrieved January 14, 2016, from www.ohsu.edu/xd/ education/library/accessmedicine

O'Brien, L., Mathieson, K., Leafman, J. \& Rice-Spearman, L. (2012). Level of stress and common coping strategies among physician assistant students. Journal of Physician Assistant Education, 23(4), 25-29.

Office of Technology Assessment. (1986). Nurse practitioners, physician assistants and certified nurse-midwives: A policy analysis (Case Study 37). Washington, DC: U.S. Government Printing Office.

Olson, K. (2011). Essentials of qualitative interviewing. Walnut Creek, CA: Left Coast Press.

Oxford Dictionaries. (n.d.). Oxford dictionary. Competence. Retrieved September 11, 2015, from http://www.oxforddictionaries.com/definition/english/competence

Padgett, D. K. (1998). Qualitative methods in social work research: Challenges and rewards. Thousand Oaks, CA: Sage.

Padgett, D. K. (Ed.). (2004). The qualitative research experience. Belmont, CA: Wadsworth/Thomson Learning.

Parker, C., \& Lewis, R. (1981). Beyond the Peter principle: Managing successful transitions. Journal of European Industrial Training, 5(6), 17-21. 
Parkerson, G. R., Broadhead, W. E., \& Tse, C-K. (1990). The health status and life satisfaction of first-year medical students. Academic Medicine, 65, 586-588.

Patient Care and Affordable Care Act, HR 3590, $111^{\text {th }}$ Cong (2010).

Pederson, R. (2010). Empathy development in medical education: A critical review. Medical Teacher, 32(7), 593-600.

Perry, J., \& Purka, N. (2008). Stress management within the undergraduate medical education curriculum. Journal of Holistic Healthcare, 5(3), 26-31.

Peter, L. J., \& Hull, R. (1969). The Peter principle: Why things always go wrong. Oxford: Morrow.

Physician Assistant Education Association. (2015). By the numbers: 30th report on physician assistant educational programs in the United States, Washington, DC: Author. doi:10.17538/btn2015.001

Prata, J., \& Gietzen, J. W. (2007). The imposter phenomenon in physician assistant graduates. Journal of Physician Assistant Education, 18(4), 33-36.

Record, J. C., McCally, M., \& Schweitzer, S. O. (1980). New health professions after a decade and a half: Delegation, productivity and costs in primary care. Journal of Health, Politics and Policy Law, 5, 470-497.

Richards, L. A. (1999, September). Reasons for optimism in agriculture: Growing your morale. Paper presented at the Philadelphia Society for Promoting Agriculture, Philadelphia, PA.

Rickelman, B. L. (2002). Demoralization as a precursor to serious depression. Journal of the American Psychiatric Nurses Association, 8(1), 9-17.

Roberts, L. W. (2010). Understanding depression and distress among medical students. Journal of the American Medical Association, 304, 1231-1233.

Robertson, D. L. (1996). Facilitating transformative learning: Attending to the dynamic of the educational helping relationship. Adult Education Quarterly, 47(1), 41-53.

Robertson, D. L. (1997). Transformative learning and transition theory: Toward developing the ability to facilitate insight. Journal on Excellence in College Teaching, 8(1), 105-125.

Roeser, R. W, Peck, S. C., \& Nasir, S. S. (2006). Self and identity processes in school: Motivation, learning and achievement. In P. A. Alexander \& P. H. Winne (Eds.), Handbook of educational psychology ( $2^{\text {nd }}$ ed.; pp. 391-392). Mahwah, NJ: Lawrence Erlbaum Associates. 
Rosenthal, S., Howard, B., Schlussel, Y. R., Herrigel, D., Smolarz, B. G., \& Kaufman, M. (2011). Humanism at heart: Preserving empathy in third-year medical students. Academic Medicine, 86(3), 350-358.

Rosenzweig, S., Reibel, D. K., Greeson, J. M., Brainard, G. C., \& Hojat, M. (2003). Mindfulness-based stress reduction lowers psychological distress in medical students. Teaching and Learning in Medicine, 15(2), 88-92.

Rubin, H. J., \& Rubin, I. S. (1995). Qualitative interviewing: The art of hearing data. Thousand Oaks, CA: Sage.

Ryder, N. B. (1965). The cohort as a concept in the study of social change. American Sociological Review, 30(6), 843-861.

Sandars, J. (2006). Transformative learning: The challenge for reflective practice. Work Based Learning in Primary Care, 4, 6-10.

Sanford, F. H., \& Holt, R. R. (1943). Psychological determinants of morale. Journal of Abnormal Psychology, 38(1), 93-95.

Sargent, A. G., \& Schlossberg, N. K. (1988). Managing adult transitions. Training and Development Journal, 42, 58-60.

Sathe, R. S. (2009). Using the cohort model in accounting education (Accounting Faculty Publications, Paper 19). Retrieved from http:ir.ssthomas.edu/ocbaccpub/19

Schlossberg, N. K. (1981). A model for analyzing human adaptation to transition. The Counseling Psychologist, 9(2), 2-18.

Schlossberg, N. K., Waters, E. B., \& Goodman, J. (1995). Counseling adults in transition (2nd ed.). New York, NY: Springer.

Schmitter, M., Liedl, M., Beck, J., \& Rammelsberg, P. (2008). Chronic stress in medical and dental education. Medical Teacher, 30, 97-99.

Schneider, D. M., \& Goldwasser, C. (1998). Be a model leader of change. Management Review, 87(3), 41.

Schor, N. F., Guillet, R., \& McAnarney, E. R. (2011). Anticipatory guidance as a principle of faculty development: Managing transition and change. Academic Medicine, 86(10), 1235-1240.

Schwabe, L., \& Wolf, O. T. (2009). Stress prompts habit behavior in humans. Journal of Neuroscience, 29(22), 7191-7198. 
Schwenk, T. L., Davis, L., \& Wimsatt, L. A. (2010). Depression, stigma and suicidal ideation in medical students. Journal of the American Medical Association, 304(11), 1181-1190.

Seashore, C. (1975). In grave danger of growing: Observation on the process of professional development. Social Change, 5(4), 1-4.

Seidman, I. (1998). Interviewing as qualitative research: A guide for researchers in education and the social sciences. New York, NY: Teachers College Press.

Shanafelt, T., D., Boone, S., Tan, L., Dyrbye, L. N., Sotile, W., Satele, D., . . . Oreskovich, M. R. (2012). Burnout and satisfaction with work-life balance among US physicians relative to the general US population. Archives of Internal Medicine, 172(18), 1377-1385.

Sharpless, J., Baldwin, N., Cook, R., Kofman, A., Morley-Fletcher, A., Slotkin, R., \& Wald, H. S. (2015). The becoming: Students' reflections on the process of professional identity formation in medical education. Academic Medicine, 90(6), $1-6$.

Silva, T., Pina e Cunha, M., Clegg, S. R., Neves, P., Rego, A., \& Rodrigues, R. A. (2013) Smells like team spirit: Opening a paradoxical black box. Human Relations, 67(3), 1-24.

Skovholt, T. M., \& Ronnestad, M., H. (2003). Struggles of the novice counselor and therapist. Journal of Career Development, 30(45), 45-58.

Sox, H. C., Jr. (1979). Quality of patient care by nurse practitioners and physician’s assistants: A ten-year perspective. Annals of Internal Medicine, 91, 459-468.

Spiegel, D. A., Smolen, R. C., \& Jonas, C. K. (1986). An examination of the relationships among interpersonal stress, morale and academic performance in male and female medical students. Social Science and Medicine, 23(11), 1157-1161.

Stafford-Clark, D. (1949). Morale and flying experience: Results of a wartime study. Journal of Mental Science, 95, 10-50.

Styles, W. M. (1993). Stress in undergraduate medical education: “The mask of relaxed brilliance.” British Journal of General Practice, 43(367), 46-47.

Tennant, M. C. (1993). Perspective transformation and adult development. Adult Education Quarterly, 44(1), 34-42.

Terry, D. J., Callan, V. J., \& Sartori, G. (1996). Employee adjustment to an organizational merger: Stress, coping and intergroup differences. Stress Medicine, $12,105-122$. 
U.S. News \& World Report. (2015). The 100 best jobs. Retrieved January 16, 2016, from http://money.usnews.com/careers/best-jobs/rankings/the-100-best-jobs

Van Gennep, A. (1960). Rites of passage. London, England: Routledge.

Van Maanen, J., \& Schein, E. H. (1979). Toward of theory of organizational socialization. Research in Organizational Behavior, 1, 209-264.

Varner, D., F., \& Foutch, B., K. (2014). Depression and burnout symptoms among Air Force family medicine providers. Journal of the American Academy of Physician Assistants, 27(5), 42-46.

Veit, C. T., \& Ware, J. E. (1983). The structure of psychological distress and well-being in general populations. Journal of Consulting and Clinical Psychology, 51(5), 730-742.

Vitaliano, P. P. (1988). A biopsychosocial model of medical student distress. Journal of Behavioral Medicine, 11, 311-331.

Vitaliano, P. P., Russo, J. R., Carr, J. E., \& Heerwagen, J. H. (1984). Medical school pressures and their relationship to anxiety. Journal of Nervous and Mental Disease, 172(12), 730-736.

Wald, H. (2015). Professional identity (trans)formation in medical education: Reflection, relationship, resilience. Academic Medicine, 90(6), 1-6.

Wear, D., Aultman, J. M., Varley, J. D., \& Zarconi, J. (2006). Making fun of patients: Medical students' perceptions and use of derogatory and cynical humor in clinical settings. Academic Medicine, 81(5), 454-462.

Woloschuk, W., Harasym, P. H., \& Temple, W. (2004). Attitude change during medical school: A cohort study. Medical Education, 38, 522-534.

Wolf, T. M., \& Kissling, G. E. (1984). Changes in life-style characteristics, health, and mood of freshman medical students. Journal of Medical Education, 59, 806-814.

Ziegler, J. L., Kanas, N., Strull, W. M., \& Bennet, N. E. (1984). A stress discussion group for medical interns. Journal of Medical Education 59, 205-207. 
APPENDIX A INFORMED CONSENT 


\section{Informed Consent}

\section{An Inquiry into the Didactic Experience of Physician Assistant Students}

You have been invited to be in this research study because you are a member of the 2014 OHSU PA class cohort. The purpose of this study is to learn more about the student experience during the didactic phase of physician assistant education and how the physician assistant program can be strengthened in the future.

This study involves participation in two separate focus group sessions. Each session will last approximately $60-90$ minutes. The initial session will be arranged during fall term of 2013 and the second session will be scheduled to take place between 4-6 weeks subsequent to the first meeting. The focus group sessions will be held in Gaines Hall at OHSU.

A total of 6-10 participants will be enrolled as subjects in this educational research. All research will be conducted at the OHSU main campus.

\section{RISKS AND DISCOMFORTS:}

There are no foreseeable risks associated with from participation in this study other than the inconvenience of attending the sessions.

\section{BENEFITS:}

You will not benefit from being in this study. However, by serving as a subject, you may help us learn about the student experience and identify ways to improve practices in the OHSU PA program. Moreover, lessons and insights from this research will be of interest to students and faculty in other physician assistant programs.

\section{CONFIDENTIALITY:}

We will not use your name or your identity for publication or publicity purposes. All of the information you provide during the interview will be kept confidential; only summaries of the interviews will be reported. A scribe will take notes during the sessions and the sessions will be audio-recorded and transcribed anonymously. You will not be personally identified in any reports of the findings. All notes and recordings associated with the sessions will be stored in a secure locked location within the OHSU PA Program office to ensure that your participation remains confidential. Only members of the research team will have access to the notes and recordings. Research data will be destroyed three years after the conclusion of this study.

Confidentiality cannot be guaranteed in a group setting; please respect each other's privacy by not repeating what was said or who attended this meeting. 


\section{PARTICIPATION:}

Participation in this study is entirely voluntary and you may withdraw at any time without penalty. You are free to choose not to serve as a research subject in this protocol for any reason. If you do elect to participate in this study, you may withdraw from the study at any time without affecting your relationship with OHSU, the investigator, the investigator's department, or your grade in any course. If you would like to report a concern with regard to participation of OHSU students or employees in this research, please contact the Portland State Human Subjects Research Review Committee at PO Box 751, Portland, Oregon, 97207 or call (503) 725-2227. If you have questions about the study itself, please contact Pat Kenney-Moore at (503) 494-1412 or kenneymp@ohsu.edu

\section{SIGNATURES:}

Your signature below indicates that you have read this entire form and that you agree to be in this study. We will provide you with a copy of this form.

Signature of Participant

Printed Name

Date 


\section{APPENDIX B}

\section{RECRUITMENT EMAIL TO STUDENTS}


Subject: An Inquiry into the Didactic Experience of Physician Assistant Students

Dear PA Student:

The experience of students during their didactic year of physician assistant education is not well studied. Little information has been collected about how students perceive their experiences during the classroom year. As a member of the class of 2014 who has completed the didactic phase, I am inviting you to participate in a focus group study that will help us understand the didactic year from the student perspective. Answers to these questions are crucial to our continued efforts to understand and improve student educational experiences. This study is being conducted by Pat Kenney-Moore in partial fulfillment of the requirements for a doctoral degree and is under the supervision of Janine Allen $\mathrm{PhD}$ in the School of Education at Portland State University.

If you agree to participate, you will be scheduled to attend two different focus group interview sessions that will be held at Gaines Hall. These sessions will take place during the fall/winter terms of 2013-2014 at a time to be determined, and will be scheduled approximately four to six weeks apart. Each session will last approximately 60 to 90 minutes. Refreshments will be provided as a small measure of appreciation for sharing your time and input to this important research.

There are no direct benefits associated with participation in this study other than contributing to knowledge about the process of physician assistant education; knowledge that may benefit future physician assistant students. There are no foreseeable burdens associated with participation in this study other than the two to three hour time commitment associated with the focus group sessions.

Although your participation is entirely voluntary, I hope you will consider participating. Your willingness or unwillingness to participate will not affect decisions involving course grades or other evaluations of your coursework, or your employment or relationship with OHSU. You may choose not to participate and can withdraw at any time.

If you choose to participate, any information that is obtained in connection with this study that can be linked to you or identify you will be kept confidential. The responses you provide will be summarized along with the responses of other students and individual responses will never be identified in any report.

Please contact Pat Kenney-Moore at 5034941412 or kenneymp@ohsu.edu if you are interested in participating in this study. If you have concerns about your participation in this study, please contact the Portland State Human Subjects Research Review Committee at PO Box 751, Portland, Oregon 97207 or (503) 725-2227. If you have questions about the study itself, please contact Pat Kenney-Moore at (503) 494-1412 or kenneymp@ohsu.edu.

Thank you,

Pat Kenney-Moore MS, PA-C (Principal Investigator)

Associate Director / Academic Coordinator

OHSU SOM Division of Physician Assistant Education

3181 SW Sam Jackson Park Rd, GH219

Portland, Oregon 97239

(503) 494-1412 


\section{APPENDIX C}

\section{OHSU LAY LANGUAGE PROTOCOL SUMMARY}




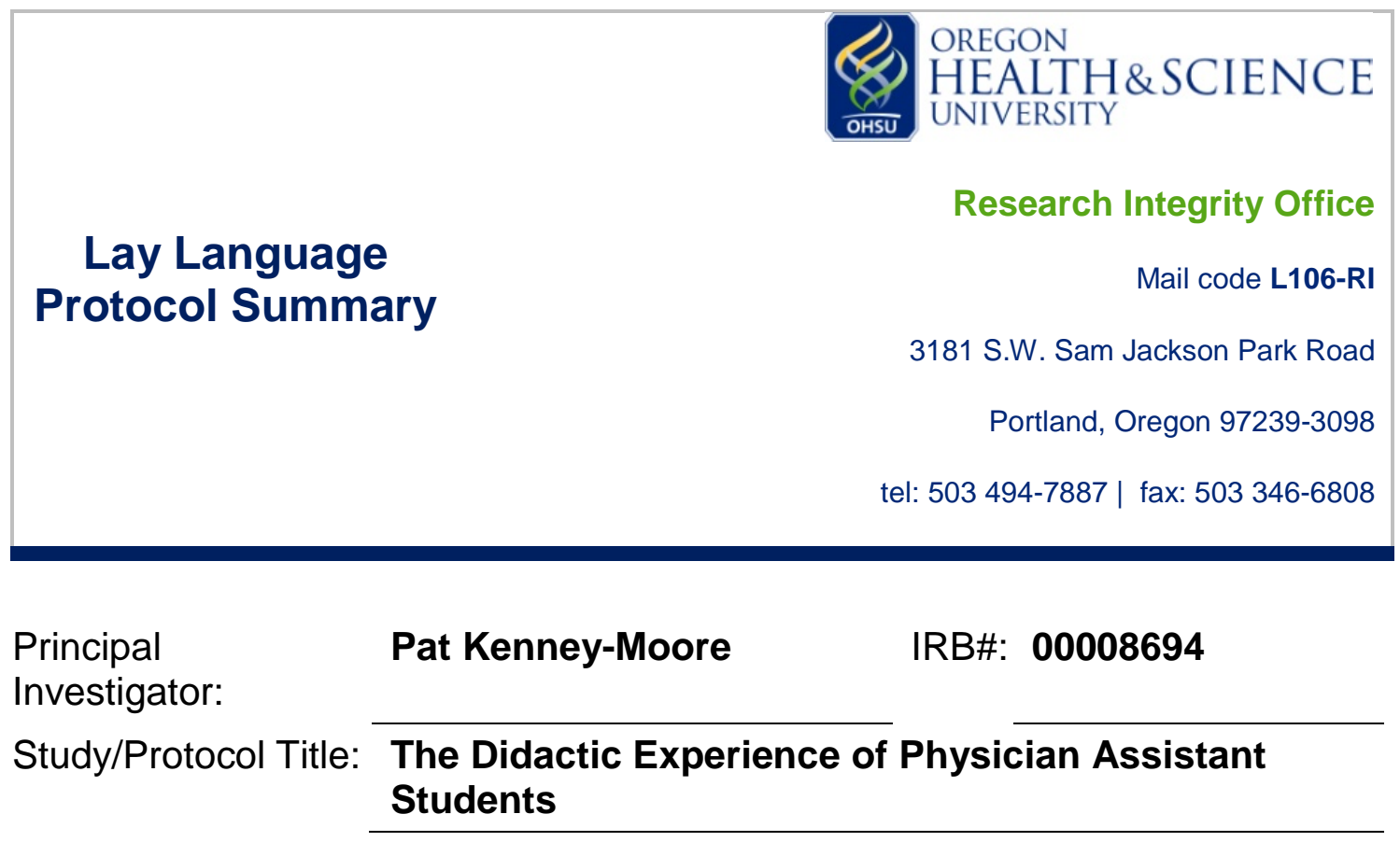

Please answer all of the following questions using lay language, similar to the language used in a consent form. Please number your responses.

1. Briefly describe the purpose of this protocol.

The purpose of this study is to gather information about the student experience during the didactic phase of the OHSU physician assistant program. After matriculation, PA students spend an intensive year in a rigorous full-time classroom-based educational experience. Currently there are little data about that experience and on its effects on student mood and morale. I am interested in investigating the student perspective of the didactic experience in order to seek improvements in the educational product, its delivery and the student experience of their engagement in that educational endeavor.

2. Briefly summarize how participants are recruited.

Participants will be recruited via email invitation. The entire cohort of 38 PA students who comprise the class of 2014 will be emailed requesting their participation in focus group sessions. Approximately 6-10 volunteers from each class cohort will ultimately be enlisted to participate. If more than 10 individuals volunteer, participants will be drawn from a hat.

3. Briefly describe the procedures subjects will undergo.

Subjects will attend two focus group meetings with members of their own cohort, each lasting approximately 60-90 minutes. Informed consent will be obtained at 
each session. The researcher will moderate a discussion about the student experience. The discussions will be audio recorded. A scribe will be in attendance and will take anonymous notes on social interactions within the group. At the conclusion of each discussion, participants will be asked to complete an anonymous written reflection on their thoughts about the focus group experience. A second cohort focus group session will be scheduled approximately 4-6 weeks after the first session to allow time for transcription of the recordings and analysis of the themes of the discussion. These themes will provide a framework for further discussion within the second focus group meeting. At the end of this second session, participants will again be asked to complete an anonymous written reflection.

4. If applicable, briefly describe survey/interview instruments used.

\section{The script for the first focus group meeting will include the following questions:}

5. Talk about your experience as a student in the didactic year of this program?

a. Did your experience change over time from when you first started the program until the end of the first year? If so, at what points did you notice the change/s?

6. What was it like being part of a cohort of students during that first year?

7. Some people believe that groups develop their own morale after being together for a time. Do you agree or not? If so, how would you describe the morale of your group?

a. Did the group morale change over time? If so, were there certain times when this was noticeable?

b. Were there ever times that the group morale differed from your own? If so, when were those times?

8. Talk about your sense of the overall experience now that the didactic year is behind you.

\section{The prompt for the written reflection will be:}

Reflecting back on the group discussion today, please take a moment to write down any additional comments that you would like to share. In your opinion, what was the most revealing part of the conversation today?

5. Briefly describe how the data will be analyzed to address the purpose of the protocol.

At the culmination of the meetings, the audio recordings will be professionally transcribed by a qualified transcriptionist resulting in formal anonymous transcripts. These session transcriptions will be reviewed by the researcher and will undergo microanalysis to identify general initial categories by which to 
conceptualize outcomes and experiences reported by students. Through a process of manual open coding, properties and dimensions of these categories will be developed as a means of distinguishing different attributes of the data, and then will be reassembled through axial coding around those identified themes. Significant statements and other components will be systematically analyzed and integrated in order to describe the phenomenon of the student experience. Hand written reflective comments will be reviewed and coded in a similar manner, providing additional perspectives from which to understand the affects of the medical education process on students. 
APPENDIX D

OHSU IRB APPROVAL MEMO 
OREGON HEALTH\&SCIENCE UNIVERSITY
Research Integrity Ofice, L106:Ri

3181 SW Sam Jackson Park Road

Portand, OR 97239.3098

(603) $494-7887$

Memo

Date: March 11, 2013

To: $\quad$ Patricia Kenney-Moore, MS

Kathryn Schuff, MD, MCR, Chair, Institutional Review Board

Elizabeth Haney, MD, Vice-Chair, Institutional Review Board

Lynn Marshall, ScD, Vice- Chair, Institutional Review Board

From: Kara Manning Drolet, PhD, Associate Director, OHSU Research Integrity Office

Andrea Johnson, JD, CIP, Regulatory Specialist, Institutional Review Board

Subject: IRB00008694, The Didactic Experience of Physician Assistant Students

\section{Special Communication for Exempt Research}

This protocol meets the requirements for Exemption from IRB review and approval in accordance with 45CFR46.101(b)[1], Research conducted in established or commonly accepted educational settings, involving normal educational practices, such as research on regular and special education instructional strategies, or research on the effectiveness of or the comparison among instructional techniques, curricula, or classroom management methods.

You are required to submit any future revisions to this research activity for prospective IRB review via Modification Request. The IRB will determine whether or not the revision affects the study's Exempt status.

A HIPAA waiver of authorization is not needed because the protocol does not involve the collection, use, or disclosure of Protected Health Information (PHI). 


\section{APPENDIX E}

\section{OHSU HUMAN SUBJECTS TRAINING}




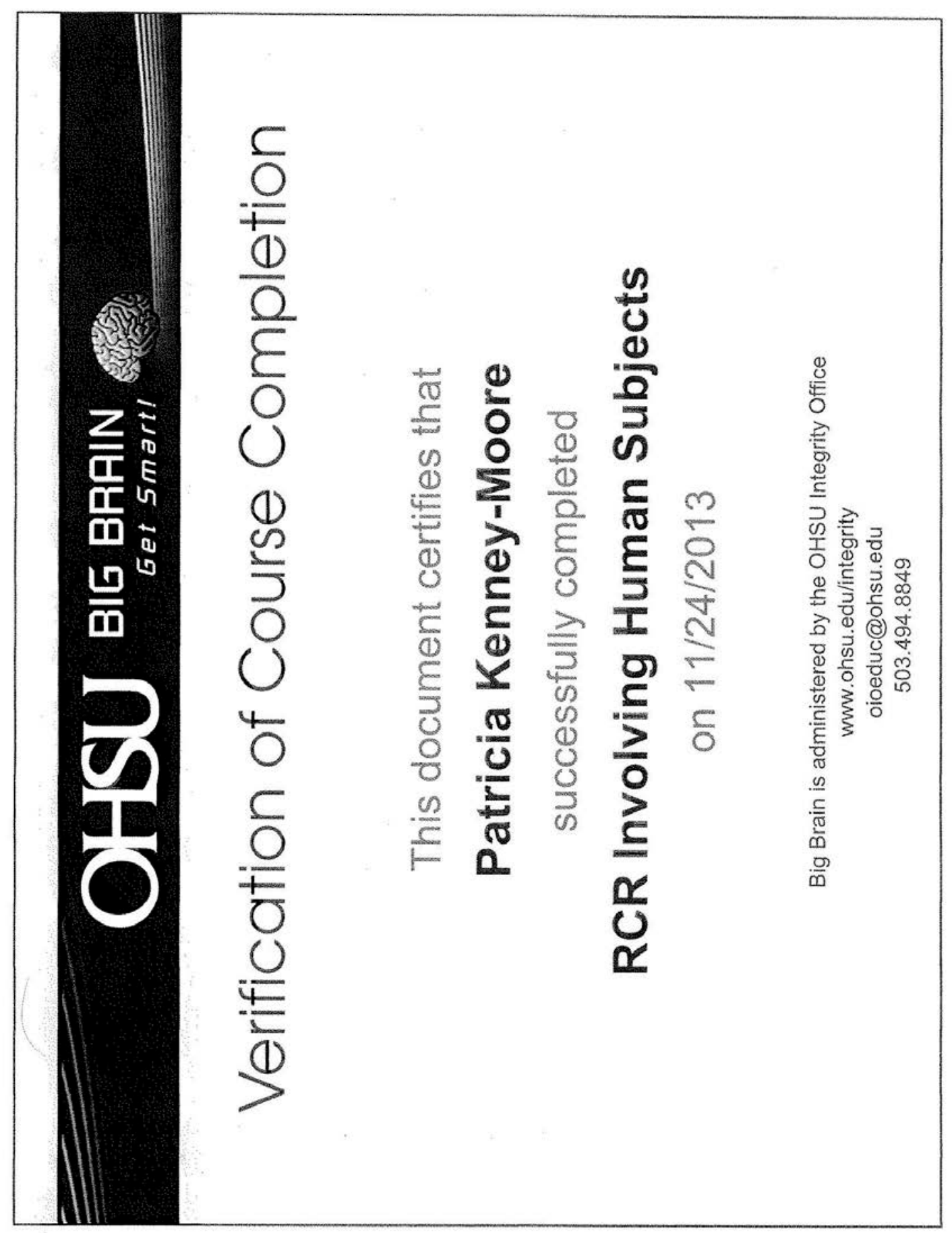




\section{APPENDIX F}

PSU INVESTIGATOR'S ASSURANCE 


\begin{abstract}
Human Subject Research Review Committee Research and Strategic Partnerships Portland State University
\end{abstract}

\title{
ff Portland State
}

Section 1: Investigator's Assurance

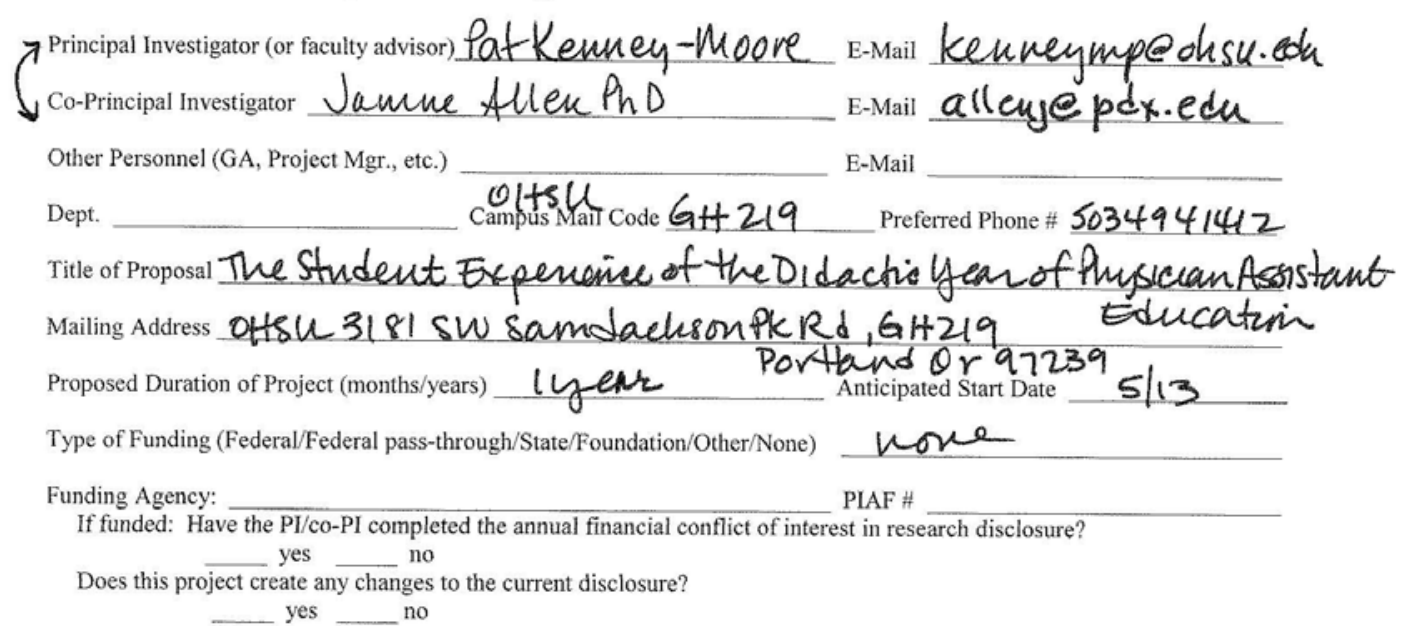

PLEASE NOTE THAT DATA COLLECTION CANNOT BEGIN UNTIL APPROVAL IS GRANTED BY HSRRC.

A. I will promptly report changes in the proposed study and any unanticipated problems involving risk to subjects, including adverse reactions, to the Human Subjects Review Committee. In case of DHHS supported activities, I will also report these problems to the Department of Health and Human Services (through the respective granting office).

B. I assure that documentary evidence of informed consent will be retained for at least three years after the proposed study has been completed or discontinued.

C. Since the Committee is obligated to review this activity at least on an annual basis, I will furnish it with a progress report approximately six weeks prior to the expiration of my project's approval.

D. I, the undersigned, will be responsible for the ethical standards of this project, and for protecting the rights and welfare of the subjects.

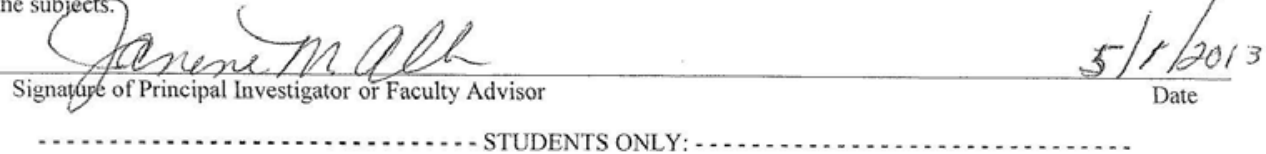

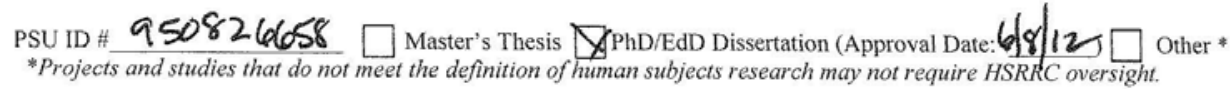

Under advisement from the above faculty member, I verify that I will conduct this research in accordance with PSU's Human Subjects Research Review Policy.

Student (print and fign) Email kenneympensusedu 
APPENDIX G

IR APPROVAL 


\section{Human Subject Research Review Committee Research and Strategic Partnerships Portland State University}

Section 1: Investigator's Assurance

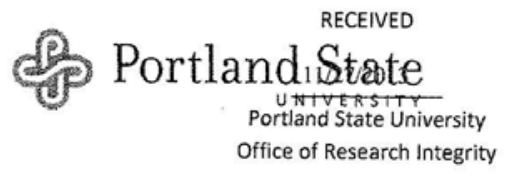

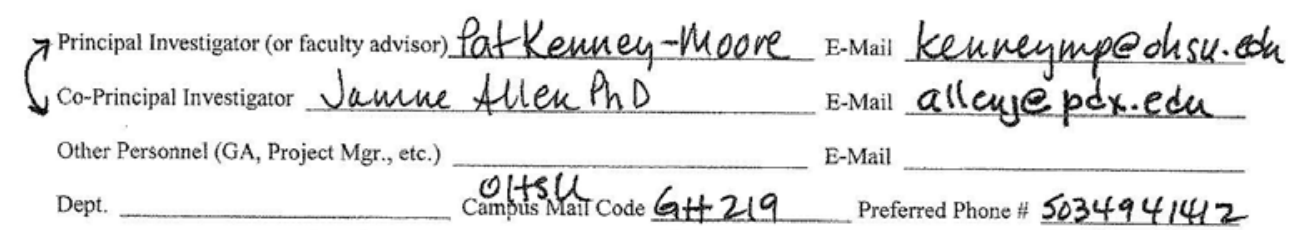

Title of Proposal The Student Expeneniee of the Didactis Year of Physician Assistant Mailing Address oftsu $3181 \mathrm{SW}$ Samdachson PK Rd, G1+219 Education Proposed Duration of Project (months/years) lyear $\quad$ Porthand Or 97239

Type of Funding (Federal/Federal pass-through/State/Foundation/Other/None) Funding Agency: wore

If funded: Have the PI/co-PI completed the annual financial conflict of interest in research disclosure? Does this project create any changes to the current disclosure? yes no

PLEASE NOTE THAT DATA COLLECTION CANNOT BEGIN UNTIL APPROVAL IS GRANTED BY HSRRC. Investigator's Assurance
A. I will promptly report changes in the proposed study and any unanticipated problems involving risk to subjects, including
adverse reactions, to the Human Subjects Review Committee. In case of DHHS supported activities, I will also report these
problems to the Department of Health and Human Services (through the respective granting office).

B. I assure that documentary evidence of informed consent will be retained for at least three years after the proposed study has been completed or discontinued.

C. Since the Committee is obligated to review this activity at least on an annual basis, I will furnish it with a progress report approximately six weeks prior to the expiration of my project's approval.

D. I, the undersigned, will be responsible for the ethical standards of this project, and for protecting the rights and welfare of the subjects.

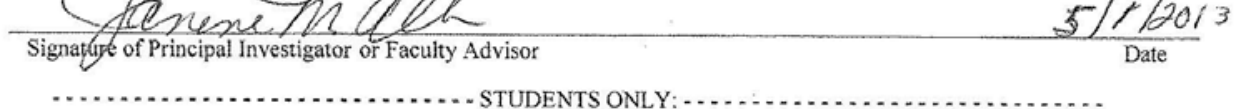

PSU ID \# $950826658 \square$ Master's Thesis $\$ PhD/EdD Dissertation (Approval Date: $6 / 8 / 12 \square$ Other *

"Projects and studies that do not meet the definition of human subjects research may not require HSRRC oversight.

Under advisement from the above faculty member, I verify that I will conduct this research in accordance with PSU's Human Subjects Research Review Policy.

Student (print and signe werey. Email kenneympeoksu.edu

Updated 5/1/2013

APPROVED

$12 / 14 / 2013-12 / 13 / 2014$

5

Portland State University

Office of Research Integrity 


\section{APPENDIX H}

IR APPROVAL MEMO 


\title{
\& Portland State
}

\author{
Post Office Box $751 \quad 503-725-2227$ tel \\ Portland, Oregon 97207-0751 503-725-8170 fax \\ Human Subjects Research Review Committee \\ hsrre@lists.pdx.edu
}

Date: December 14, 2013

To: Janine Allen / Pat Kenney-Moore

From: Karen Cellanius, HSRRC Chair

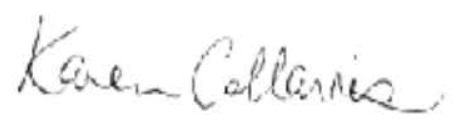

Re: HSRRC approval for your project titled, "The Student Expenience of the Didactic Year of Physician Assistant Education" HSRRC Proposal \# 132857

Approval-Expiration: December 14, 2013 - December 13, 2014

Review Type: Expedited, Categonies 6,7

In accordance with your request, the Human Subjects Research Review Committee has reviewed your proposal referenced above for compliance with PSU and DHHS policies and regulations covering the protection of human subjects. The Committee is satisfied that your provisions for protecting the rights and welfare of all subjects participating in the research are adequate, and your project is approved. Please note the following requirements:

Training: All investigators involved with this protocol must complete either the CIII or NIH human subjects training courses as soon as possible. The links are available on the Office of Research Integrity/Human Subjects website. Please provide copies of the training certificates to the ORI as soon as the training is completed.

Approval: You are approved to conduct this research study only during the period of approval cited above; and the research must be conducted according to the plans and protocol submitted (approved copy enclosed).

Consent: Signed consent is required from all participants in this study.

Changes to Protocol: Any changes in the proposed study, whether to procectures, survey instruments, consent fonms or cover letters, must be outlined and submitted to the Committee immediately. The proposed changes cannot be implemented before they have been reviewed and approved by the Committee.

Continuing Reriew: This emproval will amire on 12/13/2014, It is the investigator's responsibility to ensure that a Continuing Review Report on the status of the project is submitted to the HSRRC two months before the expiration date, and that approval of the study is kept curent. The Contimuing Review Report is available at www rsp.pdxedu/compliance_luman php and in the Office of Research and Strategic Partnerships (RSP).

Adverse Reactions and/or Unanticipated Problems: If any adverse reactions or unanticipated problems occur as a result of this study, you are required to notify the Committee immediately. If the issue is sevious, approval may be withdrawn pending an investigation by the Committee.

Completion of Study: Please notify the Committee as soon as your research has been completed Study records, including protocols and signed consent forms for each participant, must be kept by the investigator in a secure location for three years following completion of the study (or per any requirements specified by the project's funding agency).

If you have questions or concerns, please contact the Office of Research Integrity in the PSU RSP at 503-725-2227. 
APPENDIX I

IRB APPLICATION 
IRB Application

Human Subject Research Review Committee

Portland State University

Applicant: $\quad$ Pat Kenney-Moore, \#950826658

Doctoral Student, Portland State University School of Education

Advisor: Janine M. Allen Ph.D.

Professor of Education

I. Investigator's Assurance-see attached

II. Project Title and Prospectus

"Like Drinking Water Out of a Fire Hydrant"

A Transformative Medical Education Process and its Effects on Mood and Morale: An Inquiry into the Didactic Experience of Physician Assistant Students

The purpose of this study is to gather information about the student experience during the didactic phase of a professional education program for physician assistants. After matriculation, physician assistant (PA) students spend an intensive year in a rigorous full-time classroom-based educational experience before embarking on a year of apprentice-style clinical experiences. Currently there are little data about that didactic experience and on its effects on student mood and morale. I am interested in investigating the student perspective in order to seek improvements in the educational product, its delivery and ultimately the student experience during that educational endeavor. This study received IRB approval through Oregon Health \& Science University (IRB00008694) on March 11, 2013. A copy of the approval memo is attached in section XI. 
Participants will be recruited via email invitation which will be sent to the entire cohort of 38 PA students who comprise the class of 2014 with the goal of recruiting 6-10 volunteer subjects who will attend a total of two focus group meetings with other members of their own cohort, each lasting approximately 6090 minutes. Informed consent will be obtained by the researcher at the beginning of the first session. The researcher will moderate a discussion about the student experience using a focus group script. The discussions will be audio recorded. A scribe will be in attendance and will take anonymized notes on social interactions within the group. At the conclusion of the discussion, participants will be asked to complete an anonymous written reflection on their thoughts about the focus group experience and any aspect of their educational experience on which they wish to elaborate.

At the culmination of the meeting, the audio recordings will be professionally transcribed by a qualified transcriptionist resulting in formal de-identified transcript that will guide the framework for the discussion to be held during the second focus group session scheduled for four to six weeks after the initial meeting. At the conclusion of the second discussion, participants will again be asked to complete a written reflection.

Anonymous session transcriptions will be reviewed by the researcher and will undergo microanalysis to identify general initial categories by which to conceptualize common outcomes and experiences reported by students.

Through a process of manual open coding, properties and dimensions of these categories will be developed as a means of distinguishing different attributes of the data, and then will be reassembled through axial coding around those identified themes. Significant statements and other components will be systematically analyzed and integrated in order to describe the phenomenon of the student experience as elaborated by the focus group discussion. Hand written reflective comments will be reviewed and coded in a similar manner, providing additional perspectives from which to understand the effects of the medical education process on PA students.

The Lay Language Protocol Summary reviewed by the OHSU IRB is attached in Section XI.

\section{Level of Risk to Subjects}

There are no foreseeable risks incurred by subjects participating in this research project other than the inconvenience associated with attending the focus group sessions. The discussion topics are directly related to the subjects' shared daily 
experiences as students in an educational cohort, and as such represent a minimal to less-than-minimal level of risk. While every effort will be undertaken to assure participant confidentiality during and after the study by the investigator, subjects will be well known to each other and comments shared within the focus group will not be anonymous. Participants will be instructed to maintain confidentiality upon conclusion of the focus group sessions.

\section{Subject Recruitment}

Participants will be recruited via email invitation. The entire cohort of 38 PA students who comprise the class of 2014 will be emailed requesting their participation in focus group sessions. Of those responding, approximately 6-10 volunteers from the class cohort will be enlisted to participate. If more than 10 individuals volunteer, participants will be drawn from a hat. There are no exclusionary criteria and it is expected that subjects will be a mix of the genders, ages and ethnic groups represented among the class cohort. The recruitment email is attached in Section XI.

\section{Informed Consent}

Written informed consent will be obtained from all subjects by the investigator prior to the start of the initial focus group session. A copy of the signed document will be provided to participants. The informed consent document is attached in Section XI.

\section{First-Person Scenario}

"I received an email inviting me to participate in two group meetings with fellow students to talk about my experience as a physician assistant student at OHSU. I emailed the researcher to express my interest in participating and was asked to come to a focus group where I reviewed and signed an informed consent document. Once the meeting started we had a group conversation about the first year experience where we shared our viewpoints on being a student in the program. At the end of the meeting we were given the opportunity to put any additional thoughts that didn't get expressed down on paper. About 4-6 weeks later we met again and further discussed some of the ideas we brought up during the first focus group, after which we had another opportunity to write down additional thoughts. It was enjoyable to be able to debrief about my experience as an OHSU PA student and hear how others perceived their educational experience." 
VII. Potential Risks and Safeguards

There are no anticipated risks associated with participation in this study other than the inconvenience of attending the focus group sessions and the fact that confidentiality cannot be guaranteed in a group setting. Participants will be instructed about the importance of maintaining the confidentiality of the group discussion at the beginning of each focus group session. The faculty member conducting this research is not in a position of direct authority over students at this stage in their educational process, and participation is entirely optional. Should any student feel uncomfortable about sharing their thoughts about their educational experience they can elect to avoid participating in the research project.

VIII. Potential Benefits

There are no direct benefits to participants in this study other than the satisfaction of sharing the story of their educational experience among their peers. There may benefits to future PA students if this study identifies insights about the educational experience that can improve educational practices within the OHSU PA program. Any lessons and insights gleaned from this study will potentially be of interest to other PA training programs as well.

IX. Confidentiality, Records \& Distribution

Privacy and confidentiality will be strictly maintained for focus group participants in a number of ways. The doctoral student and a scribe will be the only attendees during focus groups sessions other than student participants. Participants will be well known to each other-as a result, introductions will not be necessary. Digital audio recordings done during each focus group session will not include names of participants, resulting in recorded conversations that are confidential in nature. Recordings will be stored in a password protected OHSU file accessible to the doctoral student only. These audio-recordings will provide a foundation on which to identify themes among the student experiences, as well as directing the script for the second focus group session. Recordings of the two focus group sessions will be transferred electronically to a qualified transcriptionist without identification of participants, resulting in anonymous written transcriptions. The transfer will take place through a secure OHSU password protected and confidential email process.

During the focus group sessions the scribe will take anonymized notes about social interactions during the discussions. These notes will be transcribed into a word processed document that will be housed in a password protected OHSU account held by the doctoral student. Anonymous written reflections completed 
by the participants will be word processed by the scribe prior to delivery to the doctoral student to avoid handwriting recognition and will be stored in a locked file cabinet in Gaines Hall.

While confidentiality cannot be guaranteed in a group setting, participants will be instructed at each focus group meeting that contents of discussions are not to be shared outside of the group in order to respect the privacy of fellow participants.

Students will not be personally identified in any reports developed of the findings. All notes and recordings associated with the sessions will be stored in a secure locked location within the OHSU PA Program office to ensure the confidentiality of subject participation. Only members of the research team will have access to the notes, recordings and transcriptions. All of the information gathered during the focus group interview will be kept confidential; only summaries of the interviews will be reported. Research data will be destroyed three years after the conclusion of this study via deletion of electronic files and confidential shredding of paper documents.

X. Training and Experience

The researcher has completed human subjects training through OHSU and will complete the required IRB training through Portland State. Certificate of Completion for RCR Involving Human Subjects through OHSU is attached in Section XI.

\section{Additional Documents}

Attached please find the following additional documents:

1. Informed consent

2. Recruitment email

3. OHSU Lay Language Summary: Includes the focus group script

4. OHSU IRB Approval Memo

5. OHSU Verification of Human Subjects Training

6. Scanned Investigator's Assurance 
APPENDIX $J$

\section{APPROVED PSU INVESTIGATOR'S ASSURANCE}




\section{S E C T I O N I \\ $\Delta$ Investigator's Assurance}

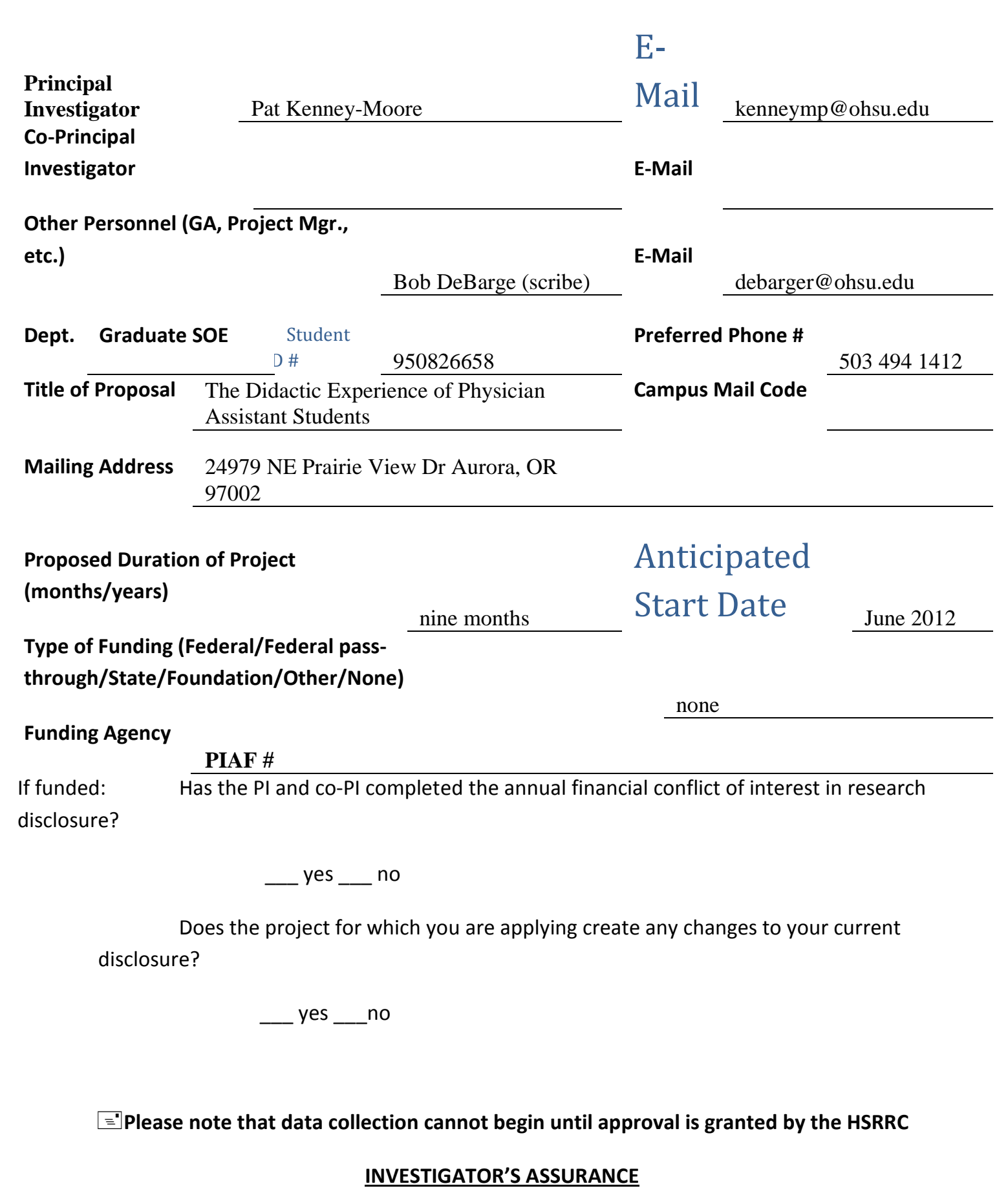


A. I will promptly report changes in the proposed study and any unanticipated problems involving risk to subjects, including adverse reactions, to the Human Subjects Review Committee. In case of DHHS supported activities, I will also report these problems to the Department of Health and Human Services (through the respective granting office).

B. I assure that documentary evidence of informed consent will be retained for at least three years after the proposed study has been completed or discontinued.

C. Since the Committee is obligated to review this activity at least on an annual basis, I will furnish it with a progress report approximately six weeks prior to the expiration of my project's approval.

D. I, the undersigned, will be responsible for the ethical standards of this project, and for protecting the rights and welfare of the subjects.

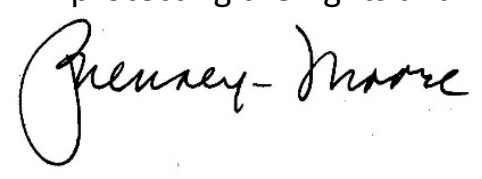

June 15, 2012

Signature of Principal Investigator

Date

I have read and approved this proposal:

Christine Cress

\begin{tabular}{llc}
\hline Department Head (PRINT) Signature & Date
\end{tabular}

GRADUATE STUDENTS ONLY:

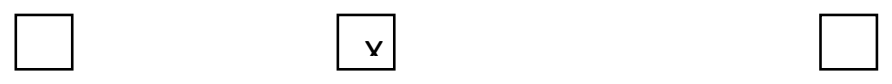

Projects and studies that do not meet the definition of

Master's Thesis Doctoral Dissertation Other human subjects research may not require HSRRC oversight; please contact us if you have questions.

PhD Committee approval date_June 8, 2012

Janine Allen

Dissertation/Thesis/Project Advisor (PRINT) Signature $\quad$ Date


APPENDIX K

OHSU PROPOSED PROJECT QUESTIONNAIRE 
$\square$ Stimulus Funding?

PROPOSED PROJECT QUESTIONNAIRE (PPQ) - PLEASE TYPE

$\mathrm{PPQ} \#$

Oregon Health \& Science University Research Development and Administration (RDA)

This form must accompany all grant/contract applications and new protocols submitted for review by X IRB or LACUC.

\begin{tabular}{|llll}
\hline GENERAL INFORMA Telphone Number Mail Code Email Address & NIH Contmons UserID \\
\hline Principal Investigator (Last name, First name, Degrees) & Telons
\end{tabular}

$5034941412 \quad$ GH219 kemneymp@ohsu.edu

Contact for questions during proposal review process Telephone Number Mail Code Email Address

$\begin{array}{llll}\text { (Lass nante, First name) Kenney-Moore, Pat } & 5034941412 & \text { GH219 } & \text { kenneymp@ohsu.edu }\end{array}$

School/Unit: School of Medicine Department: Division: Physician Assistant Education

Award Owning Org Name (Name of the org that the Award will be assigned to and that will receive F\&A credit unless otherwise specificd below):

Please see the OHSU Projectowining Org Finder 'Tool.

Will F\&A be shared by more than one department or unit? "Ifyes, indicate agreement by having each departmentunit $\quad$ Yes* No head and each internal project Pl sign this PPQ. Also include an internal budget showing the distribution of funds requested.

Project Title ( 240 characters maximum. Same as project title listed in grant or contract.)

A Retrospective Inquiry into a Transformative Cohort Medical Education Process and its Affect on Student Mood and Morale; The Didactic Experience

Project Short Title (30 characters maximum. Will be displayed in OGA. Must be unique for each OGA Project under an OGA Award)

The Didactic Experience of Physician Assistant Students

Award Short Title (30 characters maximum. Will be displayed in OGA.)

\begin{tabular}{|llll}
\hline Project Dates & Initial Budget Petiod (Next if Non-Competing) & From: & Thru: \\
(N/A for industry contracts) & Entire Proposed Project Period & From:06-27-2012 & Thru: $12-31-2012$ \\
\hline
\end{tabular}

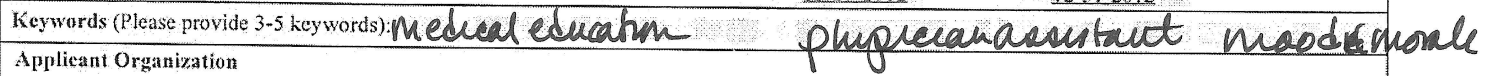

XOHSU Other*

*Ir Other, please specify pass-through organization that will issue a subcontract to OHSU:

Sponsor:

(Example: NIH, American Heart Association, Acme $\mathrm{Co}_{2}$ )

Sponsor Deadline:

Clinical Research Organization (CRO) (If applicable for an industry sponsored clinical drug / device investigation):

See Clinical Research Organization definition.

Funding Opportunity Number, Request for Proposal (RFP) \#, Request for Applieation (RFA) \#, Program Announcement (PA) \#, or URL

address for special instructions, if applicable:

ACTIVITY AND F\&A RATE INFORMATION

Does the funding agency lave a publisbed policy requiring the use of an F\&A rate that differs from OUSU's federally

negotiated rate (See OUSU's F\&A Cost Rates)? *If yes, please attach the published rate and policy of the funding agency.

This does not apply to industry sponsors.

Primary location (Building Name) where the work is being performed (See the OUSU Building List): Gaines Hall

$\%$ of work performed at this location: 100

Indicate 'Off-Campus' if the work is being performed at a non-OHSU facility.

Is the research primarily Basie or Applied? (SeeBasic/Applied Research Definitions) If the project is not
research, select N/A

TYPE OF FUNDING INFORMATIO

Funding Mechanism Click Here to Select Funding Mechanisn * If Other, please specify:

If a Program Development Account (PDA) is funding this project, please specify the PDA account number:

Grant/Contract Type Check all that apply

$\square$ New - new project not previously funded by this sponsor

$\square$ Resubmission - revised or amended version of application not funded

$\square$ Competing Renewal - competitive application for funded project Sponsor Grant”

$\square$ Amendment/Supplement - request for additional funds Sponsor Grant

Progress Report* - renewal of a funded project (i.e., non-competing renewal) Sponsor Grant\#

*If this project involves humans andlor animals, please indicate applicable IRB Protocol $\$(s)$

(if applicable)

or LACUC Protocol H(s) 